University of Tennessee Health Science Center

UTHSC Digital Commons

\title{
Genomic Instability and the Oncohistone H3K27M Drive Gliomagenesis in a Murine Model
}

Lee J. Pribyl

University of Tennessee Health Science Center

Follow this and additional works at: https://dc.uthsc.edu/dissertations

Part of the Genetic Phenomena Commons, Genetic Processes Commons, Neoplasms Commons, Neurosciences Commons, and the Physiological Processes Commons

\section{Recommended Citation}

Pribyl, Lee J. (https://orcid.org/0000-0003-0209-7814), "Genomic Instability and the Oncohistone H3K27M Drive Gliomagenesis in a Murine Model" (2020). Theses and Dissertations (ETD). Paper 543.

http://dx.doi.org/10.21007/etd.cghs.2020.0515.

This Dissertation is brought to you for free and open access by the College of Graduate Health Sciences at UTHSC Digital Commons. It has been accepted for inclusion in Theses and Dissertations (ETD) by an authorized administrator of UTHSC Digital Commons. For more information, please contact jwelch30@uthsc.edu. 


\title{
Genomic Instability and the Oncohistone H3K27M Drive Gliomagenesis in a Murine Model
}

\begin{abstract}
Maintaining genome stability is crucial for human health and it is of particular importance in neural cells during early brain development. Genome maintenance occurs at two broad stages; surveillance during DNA replication and DNA damage repair in differentiating and mature cells. Neural cells are particularly sensitive to DNA strand breaks and defective DNA damage responses can result in detrimental effects on the nervous system, including cancer. Multiple DNA repair pathways play critical roles in preventing DNA damage accumulation in stem and neural progenitor cells. The mechanisms that protect progenitor genomes also suppress DNA mutations that can result in cancer. A primary objective of this dissertation is to understand the relative contributions of key DNA repair factors that prevent tumorigenesis during cortical development. We have compared the differential effects of inhibition of homologous recombination (HR), via BRCA2-inactivation and non-homologous end-joining (NHEJ), via LIG4-inactivation towards tumorigenesis by directing their deletion specifically to early cortical progenitors using an Emx1-cre recombinase driver. We find that coincident loss of either of these repair pathways with p53 inhibition result in distinct high-grade glioma (HGG) formation resulting from elevated genome instability by DNA damage accumulation during embryogenesis. Furthermore, the presence of the oncohistone H3K27M mutation, commonly found in pediatric HGGs, enhances genome instability and accelerates cortical gliomagenesis with p53 inactivation and defective HR or NHEJ. Additionally, the H3K27M resultant gliomas showed distinctive differences in increased brain tumor penetrance and diffusion. Through RNA-sequencing and whole exome sequencing we identify upregulation of genes normally controlled by bivalent gene promoter post-translational modifications, which result in transcriptional alterations in genes important for both neural development and tumorigenesis. Mechanistically, this is done by targeting specific populations of cortical cells that are more susceptible to DNA damage and transformations that may cause additional critical mutations during a limited timeframe of early cortical development which eventually result in HGGs. We provide evidence supporting that BRCA2 functions to provide DSBR and genome stability to the early-born proliferating cortical progenitor cell population, while LIG4 provides the same function but to a lesser extent to progenitor cells and more so to post-mitotic neurons. Since, epigenetic regulation is tightly connected with neural development and differentiation, we propose the specific genes that H3K27M effects may differ depending on the time period and particular cell state from which the HGG initiates. We believe this contributes to reduced heterogeneity in glioma expression signatures with H3K27M in addition to either HR- or NHEJ-deficiency. Ultimately this work highlights the power of inducible genetically engineered mouse models as an approach to better understand the complexities of providing a connection between genome instability and gliomagenesis.
\end{abstract}

\section{Document Type}

Dissertation

\section{Degree Name}

Doctor of Philosophy (PhD)

\section{Program}

Biomedical Sciences

Research Advisor

Peter J. McKinnon, PhD 


\section{Keywords}

Cortical Development, DNA Repair, Genome Instability, Glioma, H3K27M

\section{Subject Categories}

Diseases | Genetic Phenomena | Genetic Processes | Medical Sciences | Medicine and Health Sciences | Neoplasms | Neurosciences | Physiological Processes 
DOCTOR OF PHILOSOPHY DISSERTATION

\section{Genomic Instability and the Oncohistone H3K27M Drive Gliomagenesis in a Murine Model}

Author:

Lee J. Pribyl
Advisor:

Peter J. McKinnon, PhD

A Dissertation Presented for The Graduate Studies Council of

The University of Tennessee Health Science Center

in Partial Fulfillment of the Requirements for the

Doctor of Philosophy degree from

The University of Tennessee

in

Biomedical Sciences: Cancer and Developmental Biology

College of Graduate Health Sciences

September 2020 
Copyright (C) 2020 by Lee J. Pribyl. All rights reserved. 


\section{DEDICATION}

To my wife, Samantha, for all of your support, love, dedication and sacrifice to help me accomplish my goals.

To my daughter, Harper, you bring me happiness that I may never be able to explain. 


\section{ACKNOWLEDGEMENTS}

My graduate work would not have been possible without my mentor, Dr. Peter J. McKinnon. It has been an absolute honor to work with such an expert in the field of DNA repair and neurodevelopment. I thank you for your guidance, help, support and pushing me to think as an independent scientist.

I have to thank all of the McKinnon lab members. Dr. Helen Russell helped guide me through mouse work and answer all my "basic" scientific questions. She was an excellent lab manager, a great scientist and I wish her the happiest retirement on the sea. Dr. Jingfeng Zhao, a true master of PCR and western blots. You were a wise desk mate to have and I thank you for your help with PCR and more importantly your conversations about everything in the world. Dr. Lavinia Dumitrache, I learned much from you, including one of the most difficult and frustrating experiments in the lab, the comet assay. I thank you for your patience and all of your help through the years. Dr. Youngdon Kwak, you were my first mentor in the lab as a rotation student. You first taught me ChIP-sequencing but more importantly you showed me patience and kindness and made me realize the McKinnon lab could be a second home for me. Yang Li, I owe you many thanks for almost daily checking mice for tumors and doing an enormous amount of the brain tumor collections. Your guidance and expertise in cryosectioning and immunostaining were extremely helpful and without your assistance this project wouldn't have been possible. Drs. Yuna Kim and Aditi, your advice and friendship will not be forgotten. Dr. Cristel Camacho, this project stemmed from a large part of your thoughts and work and I appreciate all the original tumor collections and analysis that you did to make this possible. Dr. Roketa Sloan Henry, I can't thank you enough for taking hundreds of tissue images on the lionheart for me and most importantly the time and effort you put into reviewing and editing my dissertation. Most notably, Suzy Downing, the amount of thanks I owe you can't be summed up here. You were critical to my success. From mouse work to tissue analysis to the last parts of gene expression analysis, you helped in almost every aspect of this project. I thank you for all of that and our conversations and friendship over the years.

I am grateful to my committee members, Drs. Tiffany Seagroves, Gerry Zambetti, Jamy Peng, Larry Pfeffer, and former member Jian Zuo, for their valued input and suggestions in guiding me through my doctoral research project. Their commitment to my development and success was irreplaceable.

Thank you to Tim Shaw and Hongjian Jin and the departments of the center for applied bioinformatics and computational biology for all of your assistance with bioinformatics analysis.

A special thanks to Drs. Suzanne Baker and Jon Larson. Your efforts in developing the H3K27M mouse and your expertise in the pediatric glioma research field provided me with indispensable help and insight into my project. 
I want to acknowledge my previous mentors and friends, specifically Dr. Melissa Geller, Dr. Tim Starr, Dr. Chris Clark and Lisa Rogers for their help in starting my career in science. You were my original science role models and without all of you, I would have never further pursued a higher level of education.

My fellow graduate students and friends: Drs. Zach Goldsmith, RJ Autry, Kirby Wallace, Jeff Rybak and future Drs. Jesse Gammons and Mark Brimble. All of your friendship and conversations over the last 6 years have made the doctoral training process go by faster than I predicted. I wish you all the best of luck and I hope our paths cross again.

The doctoral training process over the last 6 years has put me through a whirlwind of emotions. When I started this journey, I wanted my research to have a cancer biology focus and I learned very quickly that pediatric cancer was my field of research. I was extremely fortunate to have found myself in a position to do so at one of the world's most renowned children's research hospitals and in a lab that provided me all of the necessary tools and resources to succeed. My success would not have been possible without the people before me that laid the groundwork in creating and initiating the generations of the mouse models I utilized for my studies. We are living in an amazing era where technology has tremendously streamlined and accelerated the process of generating and studying cancer models. It is our job now to use these tools to our advantage in making new discoveries to develop better therapies for all different types of cancer. I owe many thanks to many people for helping me through graduate school, without you I truly couldn't have done this. Thank you.

Lastly, I want to reflect quickly on the current situation of the world. As I wrote this dissertation during a global pandemic, I, like almost everyone else, faced moments of hopelessness and despair, but if I can take one positive attribute from this time period is the promise that the scientific community brings to us. The collaborative ideas, work and effort being put forward at a remarkable pace to help develop not only a vaccine but more effective treatments for the disease, so that we can go back to our somewhat normal lives is promising in many ways. If we as a scientific and medical research community can rally together like this to treat other diseases, like cancer, I look at the future as not only hopeful but extremely promising for all of us. 


\begin{abstract}
Maintaining genome stability is crucial for human health and it is of particular importance in neural cells during early brain development. Genome maintenance occurs at two broad stages; surveillance during DNA replication and DNA damage repair in differentiating and mature cells. Neural cells are particularly sensitive to DNA strand breaks and defective DNA damage responses can result in detrimental effects on the nervous system, including cancer. Multiple DNA repair pathways play critical roles in preventing DNA damage accumulation in stem and neural progenitor cells. The mechanisms that protect progenitor genomes also suppress DNA mutations that can result in cancer. A primary objective of this dissertation is to understand the relative contributions of key DNA repair factors that prevent tumorigenesis during cortical development. We have compared the differential effects of inhibition of homologous recombination (HR), via BRCA2-inactivation and non-homologous end-joining (NHEJ), via LIG4-inactivation towards tumorigenesis by directing their deletion specifically to early cortical progenitors using an Emxl-cre recombinase driver. We find that coincident loss of either of these repair pathways with p53 inhibition result in distinct high-grade glioma (HGG) formation resulting from elevated genome instability by DNA damage accumulation during embryogenesis. Furthermore, the presence of the oncohistone H3K27M mutation, commonly found in pediatric HGGs, enhances genome instability and accelerates cortical gliomagenesis with p53 inactivation and defective HR or NHEJ. Additionally, the H3K27M resultant gliomas showed distinctive differences in increased brain tumor penetrance and diffusion. Through RNA-sequencing and whole exome sequencing we identify upregulation of genes normally controlled by bivalent gene promoter post-translational modifications, which result in transcriptional alterations in genes important for both neural development and tumorigenesis. Mechanistically, this is done by targeting specific populations of cortical cells that are more susceptible to DNA damage and transformations that may cause additional critical mutations during a limited timeframe of early cortical development which eventually result in HGGs. We provide evidence supporting that BRCA2 functions to provide DSBR and genome stability to the early-born proliferating cortical progenitor cell population, while LIG4 provides the same function but to a lesser extent to progenitor cells and more so to post-mitotic neurons. Since, epigenetic regulation is tightly connected with neural development and differentiation, we propose the specific genes that H3K27M effects may differ depending on the time period and particular cell state from which the HGG initiates. We believe this contributes to reduced heterogeneity in glioma expression signatures with H3K27M in addition to either HR- or NHEJ-deficiency. Ultimately this work highlights the power of inducible genetically engineered mouse models as an approach to better understand the complexities of providing a connection between genome instability and gliomagenesis.
\end{abstract}




\section{TABLE OF CONTENTS}

CHAPTER 1. INTRODUCTION .....................................................................................1

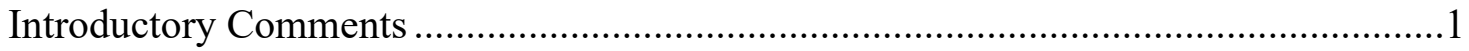

DNA Double Strand Break Repair During Cortical Development................................1

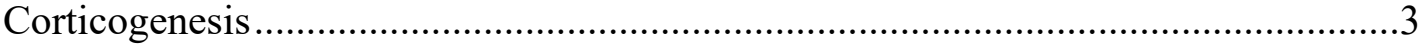

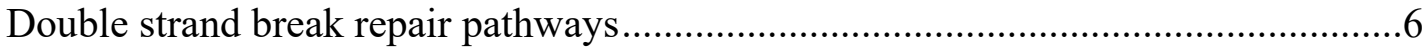

Double strand break repair deficient mouse models ............................................

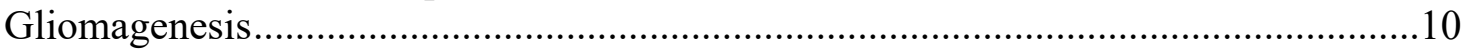

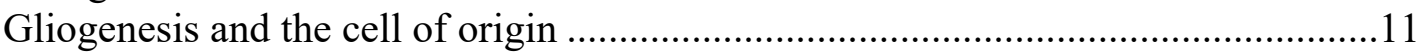

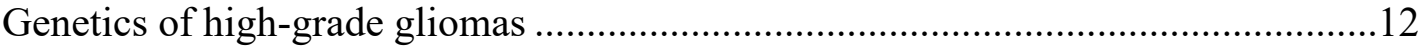

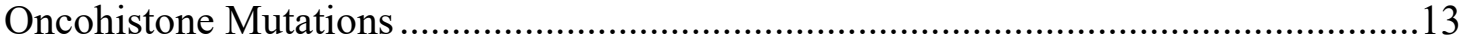

H3K27M glioma mouse models .................................................................14

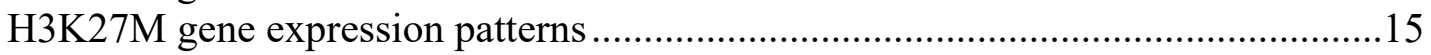

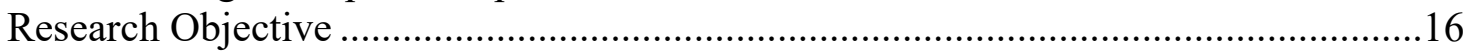

CHAPTER 2. EXPERIMENTAL METHODS .................................................17

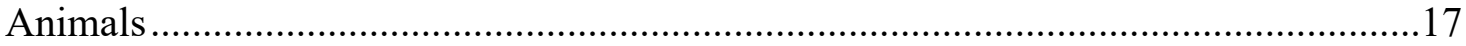

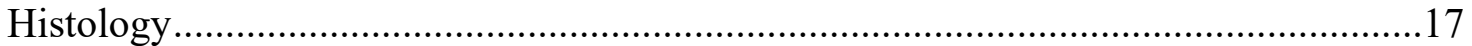

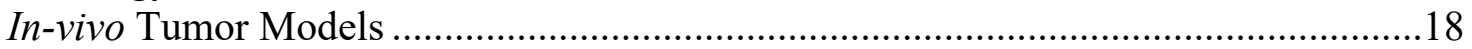

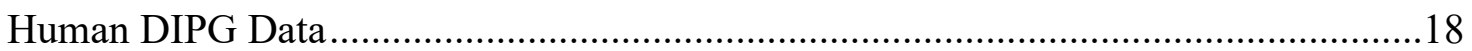

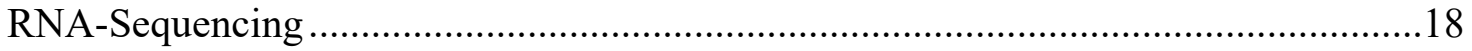

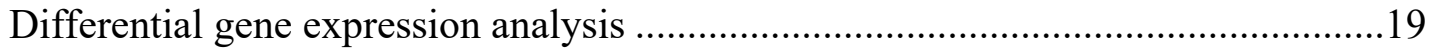

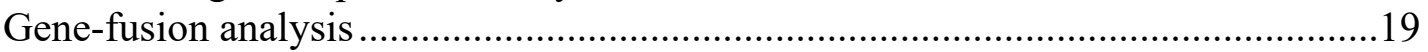

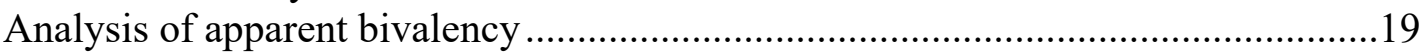

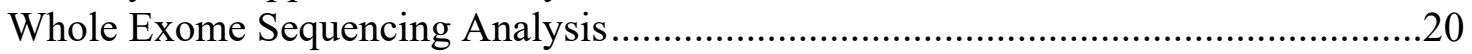

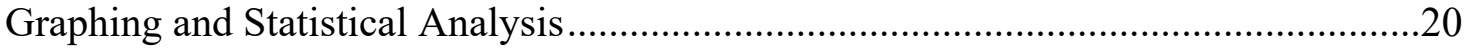

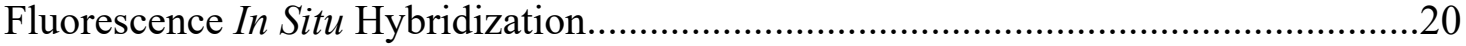

\section{CHAPTER 3. RESULTS: DOUBLE STRAND BREAK REPAIR INACTIVATION LEADS TO HIGH-GRADE GLIOMAS .......................................21}

Emx1-cre Deletion of DSBR, HR or NHEJ, Result in Cortical Cell Loss ...................21

DSBR-Deficiency and p53 Inhibition in a Mouse Neocortex Results in

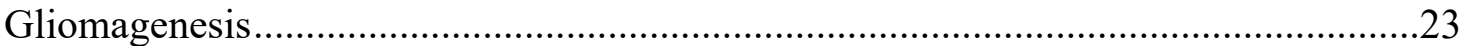

Distinct Gene Expression Profiles Characterize HGG Arising from Disabled DSBR..28

Recurrent Chromosomal Rearrangements Characterize DSBR-Deficient HGGs .........32

DSBR-Deficiency and 553 Inactivation in Neocortex Result in Selective DNA

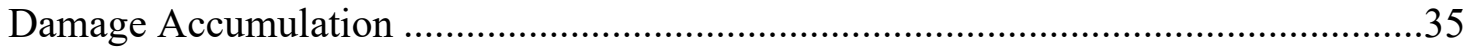

DNA Damage from DSBR-Deficiency with $p 53$ Inhibition Induces G2/M

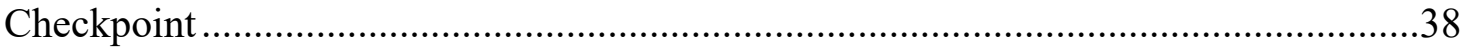




\section{CHAPTER 4. H3K27M MODULATES BIVALENT GENE EXPRESSION WITH DSBR INACTIVATION ACCELERATING GLIOMAGENESIS}

H3K27M Mutation Produces Global Loss of H3K27me3 and Increases H3K27 Acetylation in Embryos .... .42

H3K27M Accelerates Glioma Formation and Increases Tumor Diffusion... .44

H3K27M Enhances DNA damage Accumulation in the Mouse Neocortex .47

DNA Damage from H3K27M Induces G2/M Checkpoint in Cortical Progenitors ......50 H3K27M Gliomas Exhibit Unique Gene Expression Changes That Recapitulate Signatures Identified in pHGGs. .53

H3K27M Increases Glioma Cellularity and Implicates Cell of Origin Signatures .......56 H3K27M Differentiates DSBR-Deficient Gliomas by Unique Gene Expression Patterns that Effect Neural Development 59 H3K27M Alters HGG Gene Expression and Identifies Potential Gene-Fusions H3K27M Increases Expression of Bivalent Gene Promoters in HGGs .64 H3K27M Determines Cell of Origin Susceptibility to Gliomagenesis

CHAPTER 5. DISCUSSION 72

DSBR-Deficiency in Cortical Progenitor Cells .......................................................... 72

HR- and NHEJ-Deficient Tumor Gene Expression Patterns ........................................74

H3K27M Increases Genome Instability and Accelerates Gliomagenesis .....................75

H3K27M and DSBR-Deficiency Increase DNA Damage and Tumorigenesis .............77

The Role of H3K27M in Genome Instability .........................................................78

H3K27M Drives Unique Gene Expressions Between HR- and NHEJ-Deficiency.......79

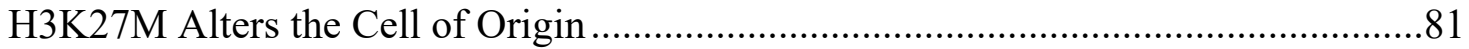

H3K27M and NHEJ-Deficiency Effect Neural Development Genes ..........................82

H3K27M and Bivalent Gene Promoters ................................................................83

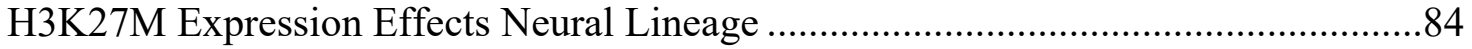

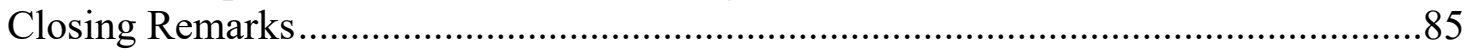

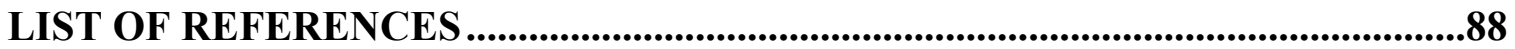

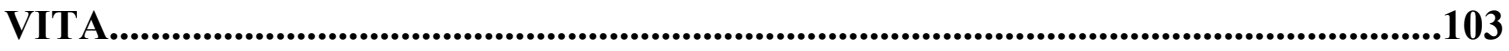




\section{LIST OF TABLES}

Table 1-1. DNA repair pathways and disruptions resulting in neuropathology..............2 


\section{LIST OF FIGURES}

Figure 1-1. DNA damage repair in early neural development. ....................................4

Figure 1-2. Mouse cortical development schematic..............................................5

Figure 1-3. DNA double strand break repair pathways. ...........................................

Figure 3-1. Emx1-cre inactivation of Brca2 or Lig4 result in cortical microcephaly......22

Figure 3-2. Emx 1-cre inactivation of Brca2 or Lig4 result in DNA damage and cortical cell loss.

Figure 3-3. DSBR-deficiency and p53 inactivation in a mouse neocortex results in gliomagenesis.

Figure 3-4. Gene expression profiles characterize HGGs arising from disabled DSBR.

Figure 3-5. Recurrent chromosomal rearrangements characterize DSBR-deficient HGGs.

Figure 3-6. DSBR-deficiency and p53 inactivation in neocortex result in selective DNA damage accumulation.

Figure 3-7. DNA damage from DSBR-deficiency induces G2/M cell cycle checkpoint.

Figure 4-1. H3K27M mutation produces global loss of H3K27me3 and increased $\mathrm{H} 3 \mathrm{~K} 27$ acetylation in embryos.

Figure 4-2. H3K27M accelerates glioma formation and increases tumor diffusion. ......45

Figure 4-3. H3K27M enhances selective DNA damage accumulation in the mouse neocortex.

Figure 4-4. DNA damage from H3K27M induces G2/M checkpoint in cortical progenitors.

Figure 4-5. H3K27M gliomas exhibit unique gene expression changes that recapitulate signatures identified in pHGGs.

Figure 4-6. H3K27M increases glioma cellularity and implicates cell of origin signatures.

Figure 4-7. H3K27M differentiates DSBR-deficient gliomas by gene expression patterns that effect neural development. 
Figure 4-8. H3K27M alters HGG gene expression causing potential gene-fusions........63

Figure 4-9. H3K27M increases bivalent gene promoters in HGGs...............................65

Figure 4-10. Addition of $\mathrm{H} 3 \mathrm{~K} 27 \mathrm{M}$ determines cell of origin susceptibility to gliomagenesis between Lig4 and Brca2 loss........................................68

Figure 5-1. Schematic of DSBR and H3K27M importance during early cortical

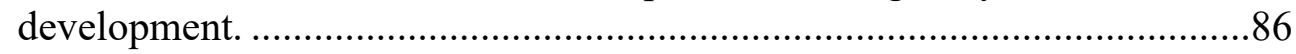




\section{LIST OF ABBREVIATIONS}

\begin{tabular}{|c|c|}
\hline$\gamma \mathrm{H} 2 \mathrm{AX}$ & phosphorylated Histone H2AX (pSer139) \\
\hline aHGG & Adult high-grade glioma \\
\hline AP & Apical progenitor cell \\
\hline aRG & Apical radial glia \\
\hline ATM & Ataxia telangiectasia, mutated \\
\hline ATR & ATM and Rad3-realted \\
\hline BER & Base excision repair \\
\hline Blm & Bloom syndrome gene \\
\hline Bmil & Proto-Oncogene, polycomb ring finger \\
\hline BMP & Bone morphogenetic protein \\
\hline BP & Basal progenitor cell \\
\hline BRCA2 & Breast cancer 2, human or mouse protein, HR component \\
\hline$B R C A 2$ & Breast cancer 2, human gene \\
\hline Brca2 & Breast cancer 2 , mouse gene \\
\hline Brca2 $2^{\text {EmxI-cre }}$ & Brca $2^{\text {loxp/loxp}} ;$ Emxl-cre \\
\hline $\operatorname{BrdU}$ & 5-Bromo-2'-deoxyuridine \\
\hline CCND & Cyclin D \\
\hline CDK & Cyclin dependent kinase \\
\hline CDKN2A & Cyclin dependent kinase inhibitor $2 \mathrm{~A}$ \\
\hline $\mathrm{CP}$ & Cortical plate \\
\hline $\mathrm{Ctrl}$ & Control \\
\hline CTX & Cortex \\
\hline DDR & DNA damage response \\
\hline DIPG & Diffuse intrinsic pontine gliomas \\
\hline DNA-PK & DNA-dependent kinase catalytic subunit \\
\hline DNA-PKcs & DNA-dependent kinase catalytic subunit catalytic subunit \\
\hline DSB & DNA double-strand break \\
\hline DSBR & Double-strand break repair \\
\hline E12.5 & Embryonic day of gestation 12.5 \\
\hline EGFR & Epidermal growth factor receptor \\
\hline $\mathrm{GO}$ & Gene ontology \\
\hline GFAP & Glial fibrillary acidic protein \\
\hline GSEA & Gene-set enrichment analysis \\
\hline FGFR & Fibroblast growth factor receptor \\
\hline FISH & Fluorescence in situ hybridization \\
\hline$H \& E$ & Hematoxylin and eosin stain \\
\hline H3K27M & Histone H3.3 Lysine $(\mathrm{K}) 27$ Methionine(M) \\
\hline H3K27M ${ }^{\text {Emxl-cre }}$ & $\mathrm{H} 3 \mathrm{~K} 27 \mathrm{M}^{\text {loxp/loxp }} ;$ Emxl-cre \\
\hline $\mathrm{HCP}$ & High-CpG-density promoters \\
\hline HGG & High-grade glioma \\
\hline HR & Homologous Recombination, DSB repair pathway \\
\hline $\mathrm{IHC}$ & Immunohistochemistry \\
\hline IR & Ionizing Radiation \\
\hline
\end{tabular}




\begin{tabular}{|c|c|}
\hline IZ & Intermediate zone \\
\hline LIG4 & DNA ligase IV, human or mouse protein, NHEJ component \\
\hline LIG4 & DNA ligase IV, human gene \\
\hline Lig4 & DNA ligase IV, mouse gene \\
\hline Lig4 ${ }^{\text {Emxl-cre }}$ & Lig4 $4^{\text {loxp/loxp }} ;$ Emx 1 -cre \\
\hline LGG & Low-grade glioma \\
\hline MAP3K1 & Mitogen-activated protein kinase kinase kinase 1 \\
\hline MMR & Mismatch repair \\
\hline MRN & Mre11, Rad50, NBS1 complex \\
\hline $\mathrm{MZ}$ & Marginal zone \\
\hline NER & Nucleotide excision repair \\
\hline NEC & Neuroepithelial (stem) cells \\
\hline NHEJ & Non-homologous end-joining, DSB repair pathway \\
\hline NSC & Neural stem cells \\
\hline $\mathrm{OPC}$ & Oligodendrocyte precursor cell \\
\hline P53 $3^{\text {Emxl-cre }}$ & p53 loxp/loxp; Emxl-cre \\
\hline PCNA & Proliferating cell nuclear antigen \\
\hline PDGFR $\alpha$ & Platelet-derived growth factor receptor, alpha \\
\hline pHGG & pediatric high-grade glioma \\
\hline Plag1 & Pleiomorphic adenoma gene 1 \\
\hline $\mathrm{PRC} 1 / 2$ & Polycomb repressive complex $1 / 2$ \\
\hline Prkdc & Protein kinase, DNA-activated, catalytic subunit gene \\
\hline PTEN & Phosphatase and tensin homolog \\
\hline PTM & Post-translational modifications \\
\hline РТP & Protein tyrosine phosphatase \\
\hline RG & Radial glial cell \\
\hline Robo & Roundabout guidance receptor 1 \\
\hline RPA & Replication protein A \\
\hline Slit & Slit guidance ligand gene \\
\hline SSB & DNA single-strand break \\
\hline SSBR & Single strand break repair \\
\hline ssDNA & Single-stranded DNA \\
\hline SVZ & Subventricular zone \\
\hline TDP1 & Tyrosyl-DNA phosphodiesterase 1 \\
\hline TopBP1 & Topoisomerase II binding protein 1 \\
\hline Vcam 1 & Vascular cell adhesion molecule 1 \\
\hline $\mathrm{VZ}$ & Ventricular zone \\
\hline WT & Wild-type \\
\hline $\mathrm{XRCC} 1 / 4$ & $\mathrm{X}$-ray repair cross complementing $1 / 4$ \\
\hline
\end{tabular}




\section{CHAPTER 1. INTRODUCTION}

\section{Introductory Comments}

Cancer is a multifaceted disease, with two of the most important contributing etiologic factors being genetic and epigenetic alterations. Over the last half century most of the focus has been on understanding the genetic side, searching for abnormal genes causing the development of human cancer. In particular, gene mutations influencing DNA repair, cell cycle regulators and cell-death pathways were found to be major genetic causes of malignancies [1]. The meaning of epigenetics has evolved over time but now more than ever an appreciation for epigenetic mechanisms are critical for our understanding of how regulation of gene expression effects cancer development. Advances in sequencing technology have been instrumental in allowing us to further examine the connections between genetic mutations and epigenetic alterations in cancer. Even with a better understanding of cancer in today's world one of the most frustrating aspects is still its unpredictability. Although it is true that cancer predominantly affects the elderly, mostly due to the accumulation of mutations over an entire lifetime, therefore it is especially surprising and emotionally difficult when it befalls in children. Thankfully pediatric cancer is scarce and pediatric brain tumors, which can be extremely detrimental, are some of the rarest [2]. Together, this dissertation presents a cumulation of work which hopes to provide further understanding of the genetic and epigenetic alterations and their roles in pediatric brain tumor development.

\section{DNA Double Strand Break Repair During Cortical Development}

Maintenance of genome stability is vital for human health and it is of particular importance in neural cells during early brain development. Genome stability is regulated by multiple DNA repair pathways that work simultaneously to ensure the integrity of DNA and defects in DNA repair can result in the development of devastating diseases, many affecting the nervous system or cancer. Table 1-1 summarizes various DNA damage response signaling pathways and associated neuropathologies resulting from disruption of genome stability $[3,4]$. These pathways include nucleotide excision repair (NER), mismatch repair (MMR), base excision repair (BER)/single strand break repair (SSBR) or double strand break repair (DSBR), which are critical in maintaining the integrity of the genome $[4,5]$. DNA double strand breaks (DSBs) are the most deleterious form of DNA damage in neural cells but are repaired primarily through homologous recombination (HR) in replicating stem/progenitor cells and nonhomologous end-joining (NHEJ) in non-replicating, differentiating cells [6,7]. A major source of endogenous DSBs come from replicative stress in the proliferating progenitors of the neocortex during early brain development [3]. The complex process of cortical development begins around six weeks after conception in humans with neurogenesis lasting until about week 18 [8]. After neurogenesis the requirements for genome maintenance markedly change in the transition to nervous system maturation. The genomes of differentiated and mature neural cells, which occupy the cortex for the rest of the its life, need to be protected 
Table 1-1. DNA repair pathways and disruptions resulting in neuropathology.

\begin{tabular}{|c|c|c|c|}
\hline DNA Repair Pathway & Main Components & DNA Damage & Neuropathology \\
\hline $\begin{array}{l}\text { Homologous } \\
\text { recombination (HR) }\end{array}$ & $\begin{array}{l}\text { ATM, ATR, MRN, } \\
\text { BRCA1/2, XRCC2, } \\
\operatorname{Rad51/54,~RPA,~} \\
\text { LIG1 }\end{array}$ & Double strand breaks & $\begin{array}{l}\text { Neurodevelopmental } \\
\text { defects/microcephaly } \\
\text { Brain tumors }\end{array}$ \\
\hline $\begin{array}{l}\text { Non-homologous end- } \\
\text { joining (NHEJ) } \\
\text { (canonical) }\end{array}$ & $\begin{array}{l}\text { ATM, ATR, } \\
\text { Ku70/ 80, 53BP1, } \\
\text { DNA-PKcs, XLF, } \\
\text { Artemis, XRCC4, } \\
\text { LIG4 }\end{array}$ & & \\
\hline Alternative-NHEJ & $\begin{array}{l}\text { PARP1, MRN, } \\
\text { CtIP, LIG1/3 }\end{array}$ & & \\
\hline Base excision repair & $\begin{array}{l}\text { XRCC1, DNA } \\
\text { Pol } \beta, \text { PARP1, APE }\end{array}$ & Single strand breaks & Neurodegeneration \\
\hline $\begin{array}{l}\text { Fanconi anemia/ } \\
\text { Homologous } \\
\text { recombination }\end{array}$ & 19 FANC proteins & DNA cross-links & $\begin{array}{l}\text { Brain tumors } \\
\text { Neurodevelopmental } \\
\text { defects/microcephaly }\end{array}$ \\
\hline $\begin{array}{l}\text { Nucleotide excision repair } \\
\text { Transcription coupled } \\
\text { repair }\end{array}$ & $\begin{array}{l}\text { XPC, PCNA, RPA, } \\
\text { LIG1/3 }\end{array}$ & $\begin{array}{l}\text { Bulky adducts/ } \\
\text { Helix-distorting lesions }\end{array}$ & $\begin{array}{l}\text { Neurodegeneration } \\
\text { Complex }\end{array}$ \\
\hline Mismatch repair & $\begin{array}{l}\text { MSH2/3/6, MLH1, } \\
\text { POLD, LIG1 }\end{array}$ & $\begin{array}{l}\text { Misincorporated bases } \\
\text { (during replication) }\end{array}$ & Brain tumors \\
\hline $\begin{array}{l}\text { MRE11/RAD50/NBS1 } \\
\text { complex } \\
\text { Ataxia telangiectasia, } \\
\text { mutated (ATM) }\end{array}$ & MRN, ATM & $\begin{array}{l}\text { Defective DNA damage } \\
\text { signaling (double strand } \\
\text { break signaling) }\end{array}$ & $\begin{array}{l}\text { Microcephaly } \\
\text { Neurodegeneration }\end{array}$ \\
\hline $\begin{array}{l}\text { Ataxia telangiectasia and } \\
\text { Rad3-realted (ATR) }\end{array}$ & ATR & Replication stress & $\begin{array}{l}\text { Neurodevelopmental } \\
\text { defects/microcephaly }\end{array}$ \\
\hline
\end{tabular}

Modified with permission from Springer Nature. McKinnon, P.J., Maintaining genome stability in the nervous system. Nat Neurosci, 2013. 16(11): p. 1523-9. 
against DNA damage [3, 4]. Damage in these cells results mainly from reactive oxygen species or transcriptional disruption breaks $[9,10]$. Hence, throughout development and maintenance of the nervous system, especially the cerebral cortex, there is a perpetual need to ensure genome integrity. The human cortex is an extremely complex and important region of the brain where even minor errors in DNA repair resulting in damaged cortical cells can be particularly detrimental [11]. Providing that the machinery for apoptosis is functional in cells that are unable to repair DSBs, p53-mediated apoptosis is the preferred pathway for DNA damage response (DDR) in cortical progenitors to eliminate damaged cells $[12,13]$. This is a delicate balance of risk versus reward the cell is programmed with, to choose death rather than risk proliferating with improperly repaired DNA that could potentially carry through development leaving opportunity for mutations resulting in severe neural consequences, like tumorigenesis [14]. Genome stability is critical for all neural development, but it is especially important for the largest and arguably the most complex portion of the human brain, the cerebral cortex. This multifaceted process of genome stability and DNA repair throughout neural development is best summarized by (Figure 1-1).

\section{Corticogenesis}

The main phase of cortical neurogenesis last about 12 weeks, with most neurons not fully maturing until almost 18 months after birth. Comparatively, the same neurogenesis period of a mouse cortex is approximately only 8 days, with maturation completing around 14 days postnatal [15]. Although the difference in neurodevelopment timing between humans and mice is vast, we must consider the comparative size. The human cortex is over 1000 times larger by area and number of neurons than that of the mouse cortex [16]. Even so, the cerebral cortex of mice make for exceptional experimental models because this region of the brain is highly conserved across mammals including the basic principles of corticogenesis and overall anatomical structure $[8,15]$. Mouse cortical development starts at embryonic day 10 (E10) in the ventricular zone (VZ) with neural stem and progenitor cells undergoing a very unique process called interkinetic nuclear migration, where the cells nuclear positioning is reflective of its particular cell cycle phase (Figure 1-2) [17-20]. This process consists of progenitor cells, starting at the apical layer of the VZ in G1 phase of the cell cycle, then migrating up to the subventricular zone (SVZ) into S-phase at which point the progenitor is considered basal because of the location. The basal progenitor eventually migrates back down to the apical surface where it goes through G2 and finally mitosis [18]. From this point the regenerative stem cell progenitor starts the process over again while the daughter cell migrates away from the $\operatorname{VZ}[17,21]$. The daughter cells use radial glial (RG) cells as scaffolding to migrate towards the SVZ to begin differentiating into neurons, astrocytes or oligodendrocytes which makes up a vast amount of the cortical cell layering process [22]. Glial cells and neurons will continue migrating and eventually mature to build the intermediate zone (IZ), then the cortical plate (CP) and lastly the marginal zone (MZ) in an innermost first and outermost last fashion [19, 21]. In the mouse, cortical development begins at E10 with the rapidly proliferating apical progenitors undergoing symmetric divisions until about E12 [19, 23]. Uniquely, these 


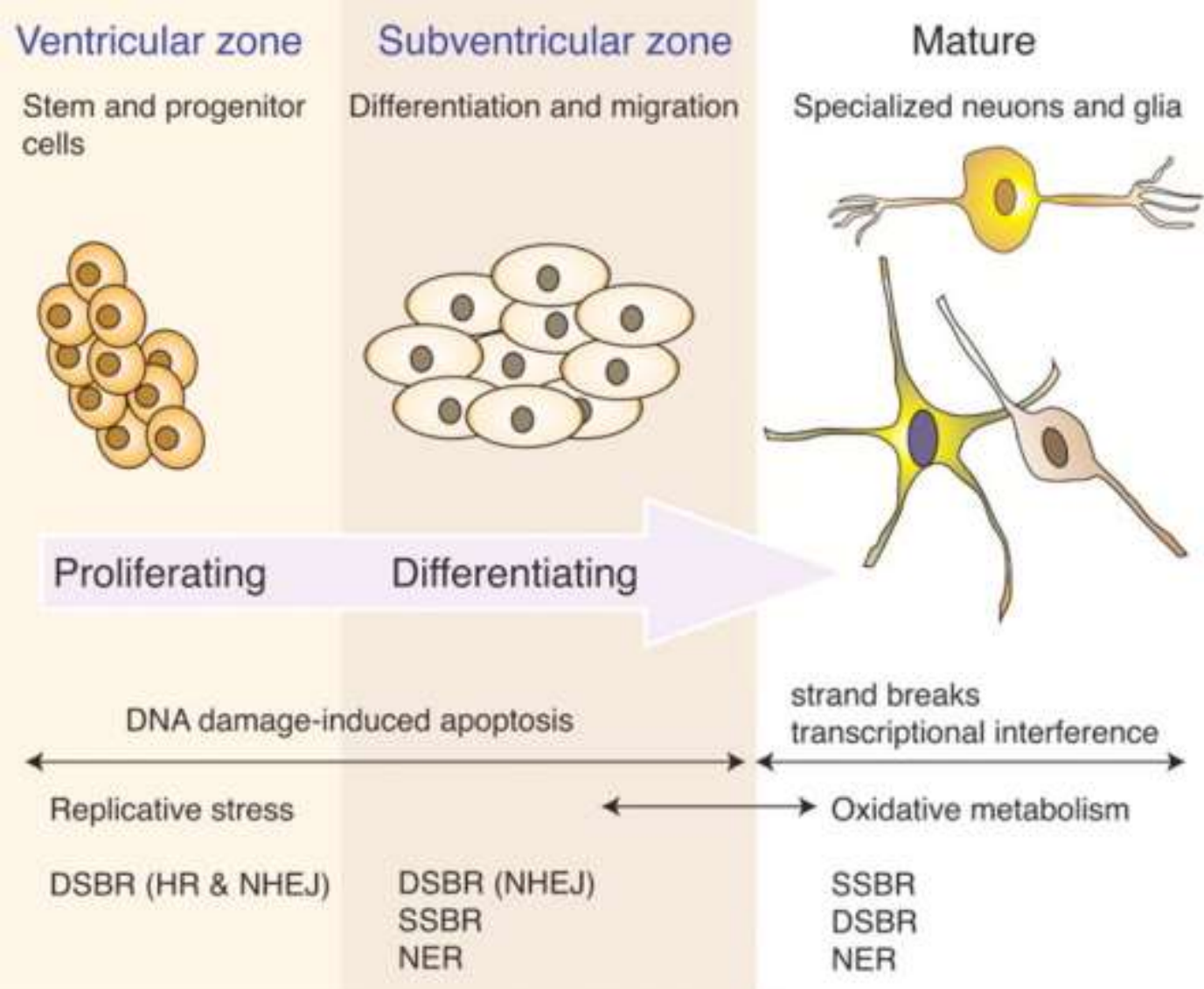

Figure 1-1. DNA damage repair in early neural development.

Neural development transpires through generalized cellular stages: proliferation, differentiation and migration. This begins at the stem and progenitor cell step that divide and start to form the ventricular zone (VZ) and some of the subventricular zone (SVZ). From this point cells undergo differentiation, migration and lastly maturation to give rise to the majority of cells in the adult brain including neurons and glia, consisting of astrocytes and oligodendrocytes. Cells are susceptible to various forms of DNA damage at the different stages of development. During proliferation, replication stress can induce DNA strand breaks that require DNA double strand break repair (DSBR) through homologous recombination (HR) or non-homologous end-joining (NHEJ). In differentiating cells DNA repair options are more restricted as HR is unavailable after cells exit the cell cycle. At this stage, NHEJ becomes the main pathway for DSBR, while other forms of DNA damage utilize nucleotide excision repair (NER) or single strand break repair (SSBR). Neural cells can be easily replaced during early development; therefore, DNA damage-induced apoptosis is a frequent result. Mature neurons and glia rely on NHEJ, SSBR, NER and various other forms of DNA repair to address DNA damage from strand breaks and reactive oxygen species from oxidative metabolism. DNA this unable to be repaired at these last stages can disrupt transcription leading to cell death.

Modified with permission from Springer Nature. McKinnon, P.J., DNA repair deficiency and neurological disease. Nat Rev Neurosci, 2009. 10(2): p. 100-12. 


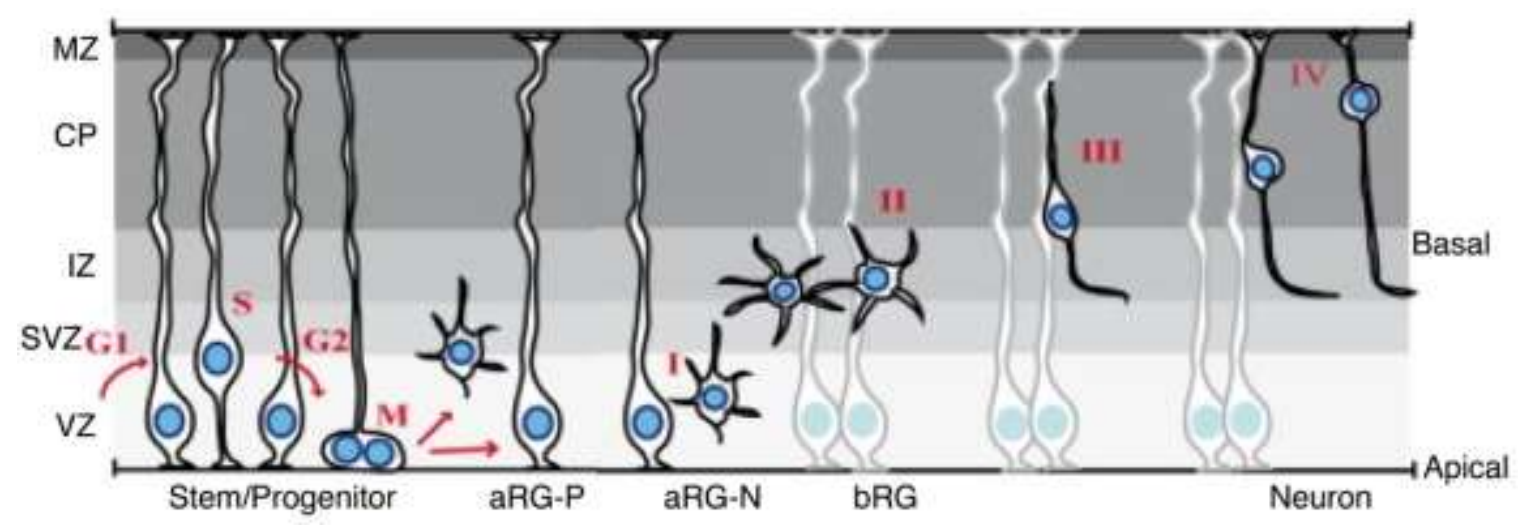

Figure 1-2. Mouse cortical development schematic.

Stem and progenitors cells initiate corticogenesis around E10 in which they undergo a process of interkinetic nuclear migration where the nuclei of these cells or radial glia (RG) cells occupy different positions along the apical-basal SVZ axis depending on the phase of the cell cycle. As cells progress through neural lineage they use RGs as scaffolding to migrate away from the apical surface and become differentiated/mature cortical neurons. Radial migration can be broken into four phases by unique cell types. Newborn neurons, like apical radial glia (aRG) committed to neurogenic lineage (aRG-N) leave the proliferative areas (aRG-P) (I) and reach the SVZ/IZ, where they attain a multipolar morphology (II). After pausing in the SVZ/IZ at the basal layer (bRG), cells migrate toward the $\mathrm{CP}$, using kinetic energy (III). At the end of their migration, cortical neurons switch to translocation of the soma (IV). During the transition to differentiation and maturation the cells exit the cell cycle.

Modified with open access permission. Azzarelli, R., F. Guillemot, and E. Pacary, Function and regulation of Rnd proteins in cortical projection neuron migration. Front Neurosci, 2015. 9: p. 19. 
cells have a longer S-phase of about 8 hours during this critical developmental time period to allow for enhance genome surveillance [24]. As the progenitor cells undergo differentiation and transition to neurogenic divisions around E13, S-phase shortens to about 2-3 hours, while the G1 phase lengthens [21, 25]. Mouse corticogenesis is not considered complete until almost post-natal day 14 , although a majority of the cortical layers are well established by E18. Decades of research on this complex developmental process has generated many unique markers for immunohistochemistry (IHC) that can be utilized to precisely identify and study the effects of specific gene mutations on the cortex [26-29]. Of particular importance to this dissertation, mutations in DSBR genes can be investigated for the effects of their loss on cortical layers like cell cycle progression, neuronal maturation and cell death throughout early brain development [14].

\section{Double strand break repair pathways}

HR and NHEJ involve multiple repair genes and associated proteins (Table 1-1 and Figure 1-3). Repair enzymes associated with the HR pathway are: ATM (Ataxia Telangiectasia, Mutated), ATR (ATM and Rad3-related), MRN complex (Mre11, Rad50, NBS1), RPA (replication protein A), BRCA1 or 2 (breast cancer type 1 or 2 susceptibility proteins) and a group of Rad51-related proteins [30-33]. HR repairs DNA by utilizing an undamaged homologous DNA strand template, sister chromatid, to replicate and replace a parallel damaged one $[33,34]$. This process starts with the MRN complex sensing a DSB and signaling for activation of ATM. ATM then autophosphorylates to form active monomers, which are recruited to the DSB to signal for cell cycle arrest through Chk1 or Chk2 to allow for DNA repair to proceed $[12,35,36]$. MRN then works to resect a DSB creating a 3' ssDNA tail, which is immediately coated with RPA. RPA is then replaced by Rad51 complex proteins, along with BRCA1/2, to assist with invasion of the sister chromatid. The proteins search for a homologous sequence on the ssDNA and once found, DNA polymerase will come in to extend the 3' end to form a Holliday Junction which is ligates the breaks together using DNA ligase I (LIG1) [33, 34]. These junctions are eventually removed by Rad54 after repair is complete. Similar to ATM, ATR can also activate MRN and the HR pathway, in situations of replication stress and stalled replication forks leading to DSBs $[11,37]$. ATR is either initiated by RAD9-RAD1HUS1 (9-1-1) complex which recognizes RPA-bound ssDNA generated by stalled replication forks, or by MRN resection of DSBs, where it forms a complex with coactivator ATR-interacting protein (ATRIP). This complex then localizes to an additional activator, topoisomerase II binding protein 1 (TopBP1) which leads to phosphorylation of downstream substrates like Chk1 and p53 to modulate cell cycle progression. HR is considered to be error-free and is especially important for replicating cells, like cortical progenitors, during the late $\mathrm{S}$ and early $\mathrm{G} 2$ phase transition. This is because of the limited access to sister chromatid, or a complementary DNA strand, during this time period of the cell cycle $[38,39]$.

In contrast, NHEJ is more error-prone because it does not use a homologous template. Instead it functions by directly modifying the two DNA end breaks so that they are compatible enough and then bluntly ligates them together, as a result some 


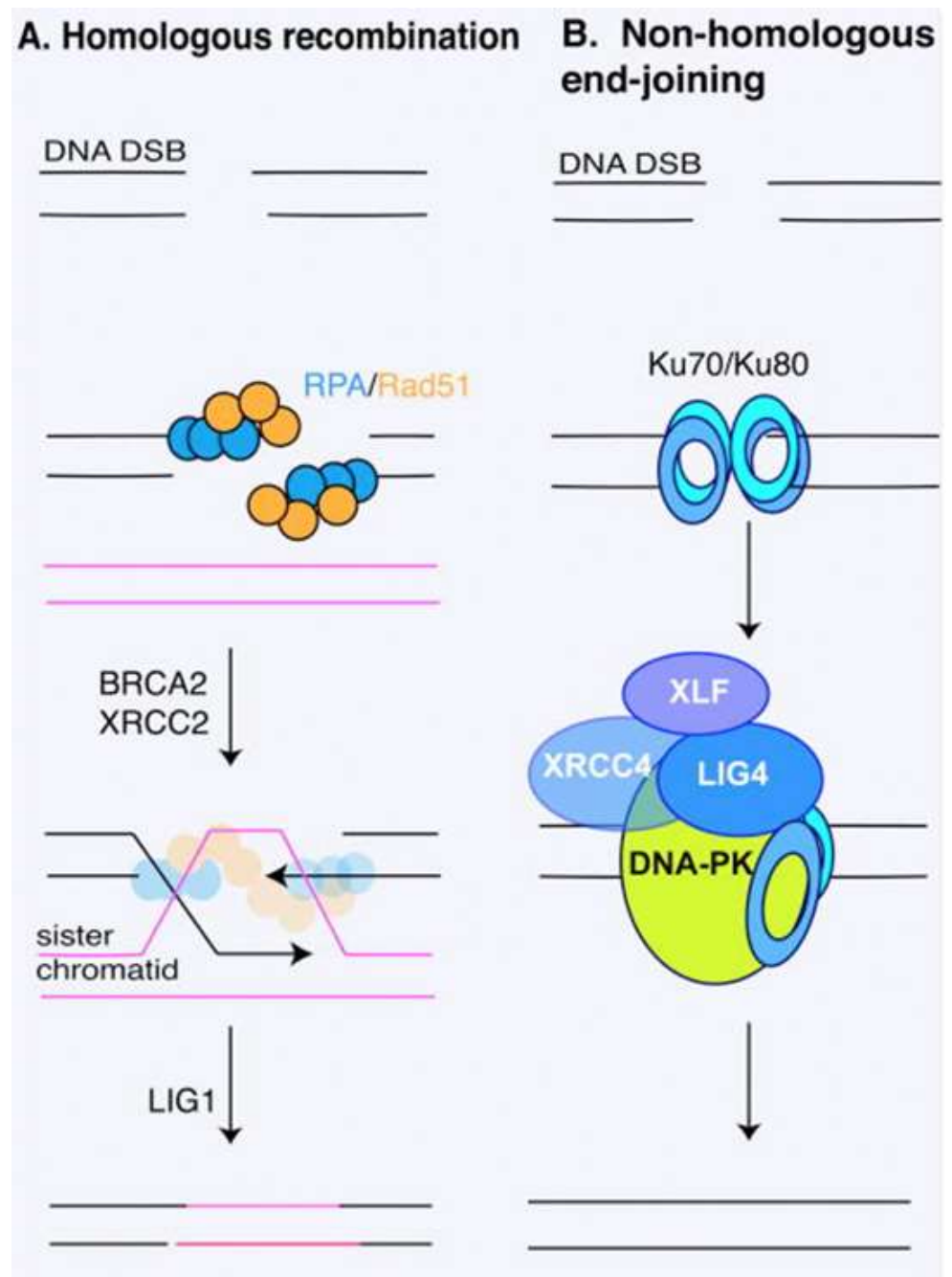

Figure 1-3. DNA double strand break repair pathways.

Double strand breaks can be repaired through two different pathways which is dependent on a cells proliferative state. (A) Homologous recombination (HR) is primarily available in the highly proliferative or replicating cells. Replication Protein A (RPA) covers the resected ends of the single stranded DNA and recruits Rad51 complex proteins along with other factors, including BRCA2 and XRCC2. These proteins work together to repair the DNA via a process where the template DNA from a sister-chromatid is incorporated into the damaged strand and acts as an error-free template. Lastly, the breaks are ligated together by DNA ligase I (LIG1). (B) Non-homologous end-joining (NHEJ) can repair DSBs in both proliferating and non-proliferating cells when needed. Heterodimeric KU70/KU80 bind broken DNA ends and recruit DNA-PK (DNA-dependent kinase catalytic subunit). Together, with XLF, XRCC4 and DNA ligase IV (LIG4), this complex works to reseal the broken DNA ends after processing from several nucleases. NHEJ is often considered an error-prone repair mechanism because some nucleotides may be lost in this process.

Modified with permission from Springer Nature. McKinnon, P.J., DNA repair deficiency and neurological disease. Nat Rev Neurosci, 2009. 10(2): p. 100-12. 
nucleotides may be lost in this process [40]. Critical repair enzymes for the NHEJ mechanism, include Ku70/80 heterodimers, DNA-PKcs (DNA-dependent protein kinase catalytic subunit), XRCC4 (X-ray repair cross complementing 4) and DNA ligase IV (LIG4) [33, 41, 42]. NHEJ is initiated by Ku70/80 binding to DSBs, which recruits DNAPKcs. Once the Ku heterodimer and DNA-PKcs join they form an active serine/threonine protein kinase complex called DNA-PK [43]. The DNA-PK complex positions the DSB ends to be merged together and recruits the exonuclease Artemis. Artemis resects DNA ends and lastly allows for XRCC4, XLF, and LIG4 to come in and complete the ligation process [44]. Additionally, there are alternative NHEJ pathways that use MRN and similar components, XRCC1 and LIG1/3, but these are less-efficient backups $[45,46]$. Although NHEJ may be an imperfect process it still repairs the DSBs and therefore the errors that may occur likely result in little consequence in non-proliferating cells [6]. NHEJ also remains an essential pathway for DSBR throughout the cell cycle, especially during G1, early S and late G2/M stages, when HR is not available [47].

While HR is the preferred and primary route of DNA repair in highly proliferative cortical progenitor cells because of its high efficiency, NHEJ acts as a backup if needed [48]. However, the role of these repair pathways effectively switch as cells differentiate and mature. NHEJ becomes the main form of DSBR in postmitotic mature neurons later in brain development because HR is essentially nonfunctional once the cells exit cell cycle [6]. Deficiencies in either DSBR pathway can result in accumulation of DNA damage $[11,49]$. If DSBs within cortical progenitors cannot be repaired by HR or NHEJ, then they will usually undergo apoptosis to eliminate the damaged cells and avoid the chance of acquiring mutations. Apoptosis is most commonly activated by ATM through Chk2/p53-signaling pathways [50]. The DNA damage signaling process has many unique steps pertaining to the specific repair pathway, however some of the initial events are common among both of them. One of the first steps is the phosphorylation event on the histone $\mathrm{H} 2 \mathrm{~A}$ variant $\mathrm{X}$ at Serine $139,(\gamma \mathrm{H} 2 \mathrm{AX})[51,52] . \gamma \mathrm{H} 2 \mathrm{AX}$ is a chromatin modification that is primarily activated at DSBs via ATM or DNA-PK, and subsequently recruits other DDR machinery to begin the repair process [53]. Identification and visualization of $\gamma \mathrm{H} 2 \mathrm{AX}$ foci, synonymous with DSBs, has been standardized through many methods including IHC, western blotting, chromatin immunoprecipitation and flow cytometry $[54,55]$.

\section{Double strand break repair deficient mouse models}

Mice are useful models to study DNA repair mutations, especially in the context of the nervous system. This is because inactivation of DNA repair factors display profound tissue specific effects $[14,56]$. Inhibition of either HR or NHEJ in mice has been shown to disrupt normal nervous system development, leading to neurodegenerative diseases, microcephaly, brain tumors and embryonic lethality [49, 57, 58]. DSBR genes are important for maintaining genome stability in numerous early developmental processes, thus germline inactivation of many of these genes result in embryonic lethality or perinatal death [59]. However, less detrimental effects are observed when gene deletion is restricted to specific organs, such as Nestin-cre which is active in neural 
progenitors. Nestin-cre is a cre recombinase that drives gene deletion throughout the entire mouse brain (Nestin-positive cells), commencing around E10-E11 [60, 61]. For example, when ATR, a PI3K-like protein kinase that is involved in DDR, is selectively inhibited with Nestin-cre it produces a substantially reduced cerebellum size, which is relatively negligible considering germline deletion is embryonically lethal $[11,62,63]$. In the context of HR, our lab has shown the loss of Brca2 alone results in overall brain size reduction with Nestin-cre, due to DNA damage induced apoptosis [58]. However, with additional germline inhibition of p53 these mice develop medulloblastomas with greater than $80 \%$ incidence. Mutations of $B R C A 2$ are not only responsible for breast and ovarian cancer, they are also found in some cases of Fanconi Anemia (FA), a rare autosomal recessive cancer susceptibility syndrome $[64,65]$. FA patients have an increased sensitivity to DNA cross-linking agents and in cases with BRCA2 mutations they display more severe phenotypes, including predisposition to medulloblastomas [66, 67]. Similarly, work from our lab has shown, conditional inactivation of Lig4 and/or Xrcc2 with null p53 again produces medulloblastomas at high frequencies (>90\%) [49]. Mutations in LIG4 (LIG4 syndrome [68]) commonly cause neurodevelopmental defects in humans, including microcephaly. Although p53 plays a somewhat indirect role in DSBR, as a response effector, it should be noted that germline $p 53$ mutations alone in mice usually do not result in medulloblastoma formation, as the mice develop lymphomas instead [49, 69-71]. Additionally, conditional inactivation of $p 53$ alone with Nestin-cre eventually result in limited medulloblastoma and glioma development however at a much later latency and far less frequency than what is found in humans with similar p53 mutations in neural tissue [49, 69]. In summary, the use of Nestin-cre to induce whole brain inactivation of DSBR genes requires concurrent inactivation of the p53 pathway and frequently results in medulloblastoma formation [72].

Similar end resulting phenotypes, like medulloblastomas, from the use of Nestincre have driven the utilization of highly tissue specific cre promoter lines to further evaluate the effects of DSBR loss on different regions of the brain, like the cortex, which can be targeted with Emxl-cre [59, 73]. Emxl-cre expresses earlier than Nestin-cre ( $\sim$ E9.5 vs. $\sim$ E10.5, respectively) and occurs primarily in the dorsal telencephalon, which develops into the neocortex $[61,73,74]$. Previous work from our lab has employed Emx 1-cre to inhibit DNA repair related genes like TopBP1, a protein linked to DNA replication and activation of ATR [75]. Remarkably, inhibition of TopBP1 by Emxl-cre resulted in a complete loss of cortical structures, while use of Nestin-cre produced a much milder phenotype. In this case, the comparative utilization of Emxl-and Nestin-cre revealed the essential role TopBP1 plays in preventing replication-associated DNA strand breaks in early cortical progenitors. In that same study, Lig4 was also conditionally inactivated by Emxl-cre, and although these mice suffered substantial DNA damage and cell loss in the cortex, which resulted in reduced cortical size, they had no obvious health issues and lived normal lifespans [75]. This was also the case for Brcal inactivation by Emx 1-cre, although in this scenario only early-born progenitors experience apoptosis while any progenitors after E16.5 were relatively unaffected [76]. This underlines the necessity of exploring different spatiotemporal gene deletion methodologies for DSBR in the context of the mouse brain, because dramatically different phenotypes can result from targeting specific genes, cell populations and developmental time periods. The mouse 
brain is a good model to study not only DNA damage repair but more importantly to study the mechanisms of human disease such as glioma development.

\section{Gliomagenesis}

Brain and nervous system tumors are the rarest forms of malignancies in adults, accounting for less than 3\% of the total U.S. cancer cases and deaths in $2019[2,77]$. Unfortunately, these are the most common solid malignancies found in children $(\sim 26 \%)$ and only second for overall pediatric cancers behind leukemia $(\sim 28 \%)[2,77]$. Adult and pediatric brain tumors may have remarkable clinical differences but they both are still extremely rare occurrences and the general etiology is unknown for most [78]. Advances in genome sequencing have allowed us to analyze thousands of matched tumor and normal samples in attempt to uncover common gene alterations among brain tumors [7982]. Many significant discoveries have come from these studies, including the identification of unique gene mutations, including EGFR, PDGFR $\alpha$ and H3K27M, shared among specific types of pediatric tumors, such as gliomas, which are distinguishable from similar morphological adult gliomas (further elaborated in following sections) [8185]. However, overall pediatric tumors were found to carry few germline mutations and tend to have less total mutations than adult malignancies, with variations dependent on the particular type of cancer [86-89]. For instance, some pediatric high-grade gliomas have very low mutational burden while others have a hypermutator phenotype [88, 90]. The genetic heterogeneity of brain tumors add to disease complexity, which drives our need to better understand the developmental processes underlying pediatric gliomagenesis to allow effective therapeutic treatment and improve patient outcomes.

Amongst the many forms of pediatric brain tumors, gliomas are the most prevalent [77]. A glioma is any tumor arising from glial cells, such as astrocytes and oligodendrocytes, which encompass the critical support network for cells in the nervous system [91, 92]. Through the advanced sequencing techniques and histological analyses, biopsies of gliomas can be categorized by the stage or grade and the cells from which the tumor arises for more accurate characterization [77]. There are four classic stages of gliomas ordered from least severe, low-grade (I and II), to most with high-grade (III and IV). Low-grade gliomas (LGGs) are most frequently diagnosed in children and accordingly have the best prognosis, while high-grade gliomas (HGGs) are predominate in adults after middle age and have worse outcomes [77]. Pediatric HGGs (pHGGs), which is a main focus of this dissertation, make up about $20 \%$ of the annual pediatric glioma cases with astrocytomas, oligodendrogliomas and ependymomas encompassing the majority [93]. HGGs in adults are primarily found in the supratentorial region of the brain, mostly composed of the cortex, while in children these gliomas are usually identified in the posterior fossa made up of the cerebellum, brainstem and diencephalon $[88,94]$. Although HGG locations may differ between human ages, the standard treatment for both are usually surgical resection, radiation and chemotherapy (temozolomide) which result in suboptimal survival results [95, 96]. Gliomas are typically characterized by the cellular composition present at diagnosis, the question of whether gliomas originally arise from that same cell type or are transformed from another 
type has been a highly contested research topic, which is commonly known as the "cell of origin" [91, 97-99]. Many studies have used both genetic analysis of human samples and novel glioma mouse models in attempt to answer this question to help better understand the mechanisms of gliomagenesis [100-102]. Potential cells of origin for gliomas include neural stem cells (NSCs), glial or astrocyte progenitor cells, oligodendrocyte progenitor cells (OPCs) and differentiated astrocytes $[91,97]$. To understand why these particular neural cells would be sources of gliomagenesis, we must also have an appreciation of how glial cells develop in the brain through gliogenesis.

\section{Gliogenesis and the cell of origin}

Corticogenesis and gliogenesis overlap in many ways and both are complex processes involving diverse cell populations beginning with stem and progenitors and eventually ending with mature neurons, astrocytes and oligodendrocytes. Identifying the correlation of the developmental steps from the origination of these cells to when and how they become tumor cells has been studied by many research groups often in mouse models [91, 97, 103]. All glial cells ascend from regenerative NSCs through a process of asymmetric divisions giving rise to intermediate progenitor cell populations, like OPCs. This first round of progenitor production begins around E12 in the mouse cortex, with a second round starting at E15 and the final round at birth $[92,104]$. Neuronal and astrocytic progenitors are usually short lived in early development with rapid differentiation into mature neurons and astrocytes [104]. Uniquely, OPCs can not only yield oligodendrocytes but they often remain as precursor cells in certain locations in the adult brain. Similarly, some NSCs can persist into adulthood, taking up residence in the SVZ and dentate gyrus [105]. With a presence in the adult brain and susceptibility to transformation due to proliferation, NSCs have emerged as plausible cell of origin candidates in multiple laboratories [100, 106, 107]. These studies showed that by using direct retroviral delivery systems of known oncogenic mutations, including $p 53$, Pten, Rb and $N f 1$, in NSCs, gliomas can reproducibly developed in mouse models [100, 101, 107].

Astrogliogenesis begins at E16-18, with the majority of astrocytes being generated in the first three weeks after birth, although some proliferating astrocytes can be found up to 52 days of age [108, 109]. Because astrocytes are highly proliferative and create large cell populations in the brain some groups are studying their possible role in gliomagenesis. The properties of astrocytes make them easy to isolate and a stable cell population to manipulate and implant into mouse models to successfully generate gliomas. Studies that have shown such in vitro models have done so by isolating astrocyte cultures from $p 53$ - or Ink4a/Arf-null mouse backgrounds and then overexpressing common oncogenes, such as MYC, RAS, AKT, PDGFR, and EGFR and implanting back into mice which result in gliomas [102, 110-116]. Further evidence supporting astrocytes as cells of origin came from a Cre-inducible model targeting glial fibrillary acidic protein (GFAP) positive cells in adult mice [117]. One study using GFAP-cre produced spontaneous astrocytomas that were reflective in location of originating from mature astrocyte populations, by mutating Pten and $p 53$ with or without $R b$ deletion [101]. However, some gliomas in this model were identified in highly 
proliferative niches which is more indicative of NSCs, which also express GFAP, suggesting that either NSCs or astrocytes could be transformed into malignant gliomas.

More recently, OPCs have become a strong contender for a cell of origin in gliomagenesis. Similar to astrocytes, a majority of oligodendrocytes come from the progenitors produced in the last round of gliogenesis after birth [104]. OPCs can also be found after development in the adult brain in slowly proliferating populations that are actually larger in total numbers than NSCs $[118,119]$. OPC expression has been identified in many human glioma samples which commonly match with the molecular signature of proneural subtype gliomas [120-122]. Many mouse models have shown OPCs capability to generate gliomas with the introduction of common human glioma mutations, including p53, PTEN, Nf1 and Ras [102, 123, 124]. Some of the best evidence supporting OPCs being a cell of origin came from a new model system developed to accurately trace cell lineages through gliomagenesis with the introduction of mutations, p53 and Nf1 in NSCs and OPCs [91, 102]. By fluorescently tracking the mutations introduced to either cell type they found that OPC gliomas were indistinguishable from NSC induced gliomas. These studies together suggest that the susceptibility to glioma formation decreases with neural lineage restriction, indicating that NSCs, progenitors and specifically OPCs may be the more likely initiating cells [98]. Although the cells of origin in glioma is still not clear, understanding this will guide the development of more effective cancer treatments.

\section{Genetics of high-grade gliomas}

Adult and pediatric gliomas are two very distinct diseases; however, they do share some overlapping characteristics, including some similar genetic mutations. One of the first mutations studied in $\mathrm{pHGG}$ were epidermal growth factor receptor $(E G F R)$ alterations because these were commonly found in adult HGGs (aHGGS) [125]. Although, EGFR variant III (EGFRvIII) mutations were identified at a much lower frequency in $\mathrm{pHGGs}$ this indicated the first signs of genetic similarities between $\mathrm{pHGG}$ and aHGGs [126, 127]. Other growth factor receptors like platelet-derived growth factor receptor alpha (PDGFR $\alpha)$ and fibroblast growth factor receptors $1 / 2 / 3(F G F R 1 / 2 / 3)$ are often amplified and/or mutated in both $\mathrm{pHGG}$ and aHGGs [111, 128-131]. In addition to these, mutations in common tumor suppressor genes are also found in HGGs, like phosphatase and tensin homolog (PTEN) and P53 [83]. Similar to EGFR, mutations of $P T E N$ are less commonly found in pHGGs with 5-15\% occurrence, compared to $25-40 \%$ in aHGGs [126]. Adult and pediatric HGGs contain diverse genomic landscapes and further observations within different types of pHGGs discovered clear differences between their own genetic etiologies.

Specific types of gliomas are often identified by their originating location. One example is diffuse intrinsic pontine gliomas (DIPGs), which are HGGs derived from the brainstem and pons that infiltrate or diffuse to surrounding normal brain tissue. DIPGs harbor additional specific mutations at slightly higher rates than other types of pHGGs, such as PDGFR $\alpha$ and p53. For instance, p53 mutations occur in about 50\% of DIPGs, 
while only in $20-35 \%$ of pediatric non-brainstem HGGs [80, 94, 132]. Besides mutations in p53, other DNA repair related gene mutations are sometimes found in $\mathrm{pHGGs,} \mathrm{such} \mathrm{as}$ ATM, ATR, BRCA2, RAD50 and several Fancomi anemia genes [80]. Other more frequent alterations in DIPGs include amplification of cyclin D genes, CCND1/2/3 (about $30 \%$ ) and mutations in $C D K N 2 A$ which encodes for the tumor suppressors p19/ARF and p16/INK4A [128, 133, 134]. Notably, a high incidence of mutations in histone $\mathrm{H} 3$ are also associated with DIPGs (see next section). DIPGs are particularly rare in both adults and children. However, brainstem HGGs occur much more frequently in children with approximately half of all pHGGs being DIPGs [77, 96]. Additionally, in younger patients the probability of fatality increases dramatically, with median survival rates less than one year after diagnosis $[80,93,96]$. The poor prognosis and low survival rates are largely due to the unique location and infiltrative nature of the tumor, making surgical resection almost impossible and radiation is only marginally effective in these children $[135,136]$. The unique and challenging characteristics of pHGGs and DIPG highlight the need to better understand the underlying genetic mechanisms of gliomagenesis to develop more effective targeted treatment strategies.

\section{Oncohistone Mutations}

Whole-genome sequencing has identified unique histone mutations as a common occurrence among subsets of HGGs. DIPGs and other pHGGs were found to frequently harbor somatic mutations in the N-terminal tail of histone H3 [81, 82]. Two of these specific missense mutations were a lysine to methionine substitution (H3K27M) and a glycine to arginine or valine $(\mathrm{H} 3 \mathrm{G} 34 \mathrm{R} / \mathrm{V})$. H3K27M mutations are predominantly found in midline or cerebellum originating tumors in younger patients, while other histone mutations such as $\mathrm{H} 3 \mathrm{G} 34 \mathrm{R}$ were identified in gliomas arising primarily in the cerebral hemispheres in slightly older patients [88]. H3K27M patients tend to have a worse prognosis and for this reason the presence of the H3K27M mutation has recently been incorporated into the World Health Organization classification as a diagnostic marker for HGGs [137]. Histone mutations have also been identified in adult cancers like leukemia and other HGGs, albeit at a much lower frequency (less than 1\%) compared to pHGGs $(\sim 50 \%)$ [80, 138-140]. Recent large collaborative efforts have molecularly analyzed a substantial amount of these rare $\mathrm{H} 3 \mathrm{~K} 27 \mathrm{M}$ gliomas revealing the biological and genetic heterogeneity of these tumors $[79,80,82,90,120,141]$. Intriguingly, these studies identified additional mutations of oncogenic pathways in the H3K27M tumors, including receptor tyrosine kinase signaling pathways (BRAF, FGFR1, ACVR1, PIK3R1 and PIK3CA) and pathways involving DNA damage repair mechanisms (TP53, ATM, PPM1D, ATRX, BLM and TOP3A). Notably, TOP3A, encoding for DNA topoisomerase III alpha, forms a complex with the bloom syndrome gene (BLM) which together facilitate the processing of HR intermediates [142, 143]. Many of these genomic aberrations represent promising therapeutic targets $[80,144]$. Taken together, the unique clinical characteristics of the pHGGs that frequently result from these histone driven mutations and the oncogenic features they exhibit, has given them the term "oncohistones". These defining oncohistone events suggest that H3K27M mutations may 
arise at different developmental time periods as well as from independent positions and cellular precursors, than tumors driven by other histone mutations, like H3G34R.

Distinctive features further support the concept that histone mutations are a dominant gain of function event in tumorigenesis. For example, all oncohistone mutations found in pHGGs are heterozygous. Additionally, although 15 genes code for histone $\mathrm{H} 3$, only 1 is mutated in any given glioma sample [81, 82]. There are three main variants of histone H3; H3.1 and H3.2 are only synthesized in chromatin during DNA replication or S phase and $\mathrm{H} 3.3$ is replication independent, processed throughout the cell cycle and is therefore found in the regions of both active gene transcription and heterochromatin $[145,146]$. Although, H3K27M only requires a single mutant allele this mutation results in a drastic loss of the repressive-associated mark, H3K27me3, throughout affected cell populations [147, 148]. This is due to the mechanism of methylation in these cells. The histone modification H3K27me3 is mechanistically deposited on chromatin by the polycomb repressive complex 2 (PRC2) to repress gene expression [149, 150]. It has recently been shown that large amounts of the H3K27M mutation are required compared to the relative quantity of PRC2 present in any given cell to yield the loss of H3K27me3 [151]. This suggests that specific neural cell populations may be more susceptible to the effects of H3K27M corresponding to the levels of PRC2. Further support of this explanation is the evidence that repressing PRC2 can prevent differentiation in embryonic stem cells resulting in a stem-like state [152]. One hypothesis is that loss of $\mathrm{H} 3 \mathrm{~K} 27 \mathrm{me} 3$ may reset potential cell of origin populations like neural precursor cells or OPCs back to an earlier stem cell like state, leading to altered cell identity [153].

\section{H3K27M glioma mouse models}

Recent studies using murine models have employed various strategies to mimic the H3K27M oncohistone effect to induce HGGs, including in utero electroporation and avian retroviral vector systems [154-158]. Although these models successfully induce gliomas and some HGGs they have limitations. First, they all require additional oncogenic drivers because H3K27M expression alone does not generate tumors. Another caveat is the source of gene activation/inactivation in these mouse models, including varying levels of exogenous $\mathrm{H} 3 \mathrm{~K} 27 \mathrm{M}$ overexpression using viral transduction.

Furthermore, the procedures are complex with in utero electroporation, in vivo retroviral transductions and/or pre- and post-natal embryonic injections being required. Therefore, these models do not always recapitulate the spontaneous and endogenous formation properties of pediatric gliomas. A more efficient model for gliomagenesis research was recently developed containing a conditional knock-in allele of the $H 3 f 3 a \mathrm{H} 3 \mathrm{~K} 27 \mathrm{M}$ mutation $[159,160]$. This model allows conditional activation with the inducible histone mutation from the endogenous locus which is controlled by a Cre recombinase-activated promotor. Using Nestin-creER for gene activation at postnatal days 0 and 1, which targets a later subset of neural stem and progenitor cells, mice effectively develop DIPGs with high penetrance in combination with PDGFR $\alpha$ activation and p53 inhibition, which closely models mutations found in pHGGs. pHGGs like DIPG are almost always a 
combination of mutations involving but not limited to growth factor receptors, epigenetic modifications and tumor suppressors $[90,139,161]$. Since p53 mutations occur in about half of pHGGs, with most being found in cerebral hemispheres, concomitant inactivation of the p53 pathway is pertinent in modeling these types of brain tumors in mice $[49,80$, 94]. The location and degree of glioma severity in this model displays one of the most highly replicative examples of $\mathrm{pHGG}$ in a murine setting. One consideration in working with this model is that spontaneous H3K27M activation using Nestin-cre produced neonatal lethality, again highlighting the complications of creating a true endogenous HGG murine model; it requires restriction of spatial and temporal expression. Although it is unknown why the combination of $\mathrm{H} 3 \mathrm{~K} 27 \mathrm{M}$ and Nestin-cre leads to neonatal death, it is clear that the targeted neural cell population and timing of gene activation plays an important role in this. More recently the inverse relationship of H3K27M mutation, where H3K27M is depleted, has been studied using mouse xenografts to examine the effect on tumor growth compared to an isogenic HGG setting [150, 162, 163]. The loss of H3K27M by nonsilencing shRNA in patient-derived xenograft models decreased tumor formation and increased survival [162], further supporting the detrimental significance of this oncohistone in gliomagenesis.

\section{H3K27M gene expression patterns}

Sequencing and expression data from many studies suggest H3K27M is associated with perturbing neural lineage differentiation, including comparable cellular subsets to other HGGs such as OPCs, cycling cells and astrocyte- and oligodendrocytelike cells $[120,155,160]$. While the variants H3.1- and H3.3K27M both drive DIPG formation, gene expression analysis of tumors carrying each suggest that $\mathrm{H} 3.1$ tumors are more astroglial and mesenchymal in nature, whereas $\mathrm{H} 3.3$ are further oligodendrocytic and proneural [164, 165]. The discovery of bivalent gene promoters brought about further gene expression scrutiny to the H3K27M mutation because of its loss of the H3K27me 3 mark [166]. A bivalent promoter is considered any nucleosome that has both H3K27me3 and $\mathrm{H} 3 \mathrm{~K} 4 \mathrm{me} 3$, repressive- and activating-associated marks, respectively. The Baker lab discovered that H3WT tumors had about $10 \%$ of the total gene promoters with both bivalent marks in their mouse model [160]. Strikingly, more than 50\% of promoters of genes that were upregulated in H3K27M gliomas were considered bivalent in the H3WT samples. This suggests that the loss of $\mathrm{H} 3 \mathrm{~K} 27 \mathrm{me} 3$ on a poised bivalent gene promotor, due to the $\mathrm{H} 3 \mathrm{~K} 27 \mathrm{M}$ mutation, results in an overall upregulation of gene expression as long as $\mathrm{H} 3 \mathrm{~K} 4 \mathrm{me} 3$ is present for activation. Another activation-associated histone modification, $\mathrm{H} 3 \mathrm{~K} 27$ acetylation $(\mathrm{H} 3 \mathrm{~K} 27 \mathrm{ac}$ ), has been reported to have increased expression in H3K27M tumors [149, 151, 163]. Specifically, the increase in H3K27ac at enhancers and super-enhancers, large clusters of enhancers, in HGGs suggests this mutation may be driving gene expression in putative cell of origin populations, like OPCs $[167,168]$. Although it has been shown that the H3K27M mutation undoubtedly contributes to brain tumor development, many of the underlying mechanisms of its function remain unclear. 


\section{Research Objective}

This dissertation investigates the effects of inhibiting DNA DSBR pathways via loss of either BRCA2 (HR) or LIG4 (NHEJ) with associated $p 53$ inactivation during the earliest stages of cortical development in a physiological setting. The subsequent chapter analyzes an innovative conditional knock-in H3K27M mouse (described above) to examine the combinatorial effect of oncohistone mutations and DSBR-deficiency on tumorigenesis in the cortex. Additionally, my studies have allowed us to further explore the connections between H3K27M and genome stability. We hypothesized that the oncohistone mutation affects the chromatin conformation state leading to increased DNA damage susceptibility as part of the mechanism leading to gliomagenesis. Collectively, these studies are the first to show an endogenous, spontaneous, cortical high-grade glioma murine model involving H3K27M alteration. Further, our data highlight the dynamic role DNA DSBR plays during early corticogenesis in preventing genomic instability and gliomagenesis. 


\title{
CHAPTER 2. EXPERIMENTAL METHODS
}

\begin{abstract}
Animals
Generation and genotyping of the conditional $H 3 f 3 a^{L S L-K 27 M-T a g / 4}, B r c a^{\text {LoxPLLoxP }}$, Lig4 LoxP/LoxP,$p 53^{\text {LoxP/LoxP }}$ and Emxl-cre mouse lines have been previously described [12, $36,49,71,159,160,169]$. The generation of the $H 3 f 3 a^{L S L-K 27 M-T a g /+}$ mouse has also been thoroughly described [159]. Brca2-floxed mice (exon 11) [71] were previously obtained from the MMHCC repository at the NIH. Lig4-floxed mice contain LoxP sites surrounding the single Lig4 coding exon and was generated as described [49]. Emxl-cre

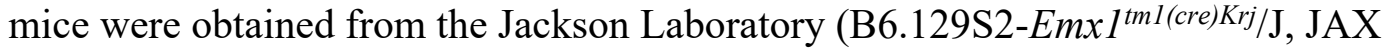
\#005628). We used conditional Emxl-cre recombinase activation/deletion to restrict effected brain region to the mouse cortex (to avoid medulloblastoma formation that occur when Nestin-cre is used [49, 58]). Mice of either sex were used for experiments. Heterozygous mice for BrCa2, Lig4, p53 and/or any combination lacking Emxl-cre were considered control subjects as full conditional gene inactivation was not present.

Animals were housed in an AAALAC-accredited facility with a 12-hour day/night cycle. A maximum of five adult animals per cage were allowed. All animal experiments were carried out in accordance with US National Institutes of Health regulations and were approved by the St. Jude Children's Research Hospital animal care and use committee (IACUC).
\end{abstract}

\section{Histology}

Mice were perfused transcardially with $4 \%$ PFA while embryos were drop-fixed in $4 \%$ PFA, and tissues were cryoprotected in 25\% PBS-buffered sucrose solution, embedded in optimal cutting temperature (O.C.T) compound, and sectioned sagittally at $10 \mu \mathrm{m}$ using an HM525 NX cryostat (Thermo Scientific). Immunohistochemistry was performed after antigen retrieval as described previously [75]. The following antibodies were used: anti-active caspase-3 (1:500; BD Biosciences, \#559565), anti-phosphoH2AX-Ser-139 (1:200; Cell Signaling Technology, \#2577L), anti-phospho-H3-Ser-10 (1:500; Cell Signaling Technology, \#9701L), PCNA (1:500; Santa Cruz, \#SC-56), antiTuj1 (1:500; Covance Research Products, \#MMS-435P), anti-Tbr2 (1:200; Abcam \#23345), H3K27me3 (1:200; Cell Signaling, \#9733), PDGFRa (1:200; Cell Signaling, \#5241), Olig2 (1:500; Millipore, \#AB9610), H3K27acetylation (1:750; Active Motif, \#39685), GFAP (1:500; Sigma, \#G3893). Immunostaining of active caspase-3 H3K27me3 and H3K27acetylation was visualized with a VIP substrate kit (Vector Laboratories, \#SK-4600) and biotinylated secondary antibody and avidin-biotin complex (Vectastain Elite kit; Vector Laboratories, \#PK-6100). Sections were counterstained with $0.1 \%$ methyl green (Vector Laboratories), dehydrated, and mounted with DPX (Fluka). For fluorescence, FITC- or Cy3-conjugated secondary antibody (Jackson Immunologicals) were used and counterstained with DAPI (Vector Laboratories, \#H- 
1200) or propidium iodide (Vector Laboratories, \#H-1300). TUNEL staining was done using the ApopTag system (Millipore Bioscience Research Reagents).

For BrdU incorporation assays, pregnant mice at E15.5 were injected (60 $\mu \mathrm{g}$ per g body weight), and $2 \mathrm{~h}$ after injection, embryos were drop-fixed in 4\% PFA and processed for cryosectioning and anti-BrdU (1:200, Abcam, \#ab6326-250) immunostaining was assessed. Apoptosis was measured by caspase-3 staining. Hematoxylin and eosin staining was also performed using a standard protocol [170]. At least three independent tissue samples from each genotype was used for all experiments and analyses.

Quantification of $\gamma \mathrm{H} 2 \mathrm{AX}$ foci, $\mathrm{Tbr} 2$ and $\mathrm{BrdU}$ positive cells were performed using the NIH ImageJ processing program. Quantification of PCNA, GFAP and Olig2 positive tumor cells were performed using Biotek Gen5 data analysis software.

\section{In-vivo Tumor Models}

Cohorts of mice were aged and monitored for brain tumor symptoms. Symptoms included but not limited to; slow, weak or intermittent breathing, decreased movement, dull, slouched, tottering movements or lateral position, decreased weight change, roughened fur, and decreased or no flight reaction. When moribund, mice were euthanized. The brain was carefully dissected, a portion of tumor was snap frozen and the remaining tissue was fixed with $10 \%$ formalin and kept at $4{ }^{\circ} \mathrm{C}$. Snap frozen tissue was used for RNA-sequencing and formalin fixed tissue was used for histology. Since our experimental mice harbor a mixed strain background and different cohorts may produce slightly different results, some mice were euthanized due to other symptoms such as enlarged clavicle masses and submandibular cervical lymph node enlargements.

\section{Human DIPG Data}

Gene expression data from human DIPGs are from a previously published dataset [90]. These datasets were used as comparisons for mouse tumor expression data, especially for chromosomal rearrangements, gene-fusions and gene amplification comparison as described below. Informed consent for specimen analysis was obtained under protocols approved by the St. Jude Children's Research Hospital Institutional Review Boards.

\section{RNA-Sequencing}

Total RNA was isolated from tissue with TRIzol (Invitrogen) and library preparation using TruSeq Stranded Total RNA Kit (Illumina): Double stranded cDNA fragments were ligated with Illumina paired end adaptors, followed by size selection ( 200bp) and libraries were analyzed by HiSeq 2000 sequencing systems (Illumina). Unsupervised hierarchical clustering was used to generate the top 1,000 most variable 
genes, which were selected using a median absolute deviation score. Statistical analyses and visualizations were performed using Partek Genomics Suite 6.6 and Stats MP/11.2 software. RNA-seq reads were aligned to UCSC mouse reference genome $\mathrm{mm} 9$ assembly using STAR software [171]. GSEA for gene set, H3K27M human tumor sample (GSE34824), was taken from Schwartzentruber et al. [82] and identified in the compiled "Gene Perturbations from Geo Up" in Enrichr.

\section{Differential gene expression analysis}

FASTQ sequences are mapped to the University of California Santa Cruz mouse reference genome (mm9) and the gene-level fragments per kilobase of transcript per million (FPKM) values and counts per million (CPM) computed for genome-wide analysis of differentially expressed genes via LIMMA in R 3.6.0. Read count for each gene was obtained with HT-seq [172]. Gene set enrichment analyses were carried out using GSEA with MSigDB [173]. Specifically, we used "-metric signal2noise -set_min 4 -permute gene_set". GSEA FDR 25\% cutoff was applied to examine enriched gene sets. ssGSEA was carried out using a non-parametric and unsupervised method; Gene Set Variation Analysis (GSVA) package in $r$ [174]. False discovery rates (FDR) were set at $<0.05$. Unpaired student t-tests were performed for significance analysis. LogFC cutoffs were $<0.75$ and p-values were $<0.05$. Enrichr from Icahn School of Medicine at Mount Sinai was used for evaluating differentially expressed gene targets associated with pathways $[175,176]$. The Gene Ontology Consortium was used in addition to Enrichr for pathway analysis. Much of the bioinformatic analysis of sequence data was performed in a collaborative effort between our laboratory including, Susanna Downing, along with assistance from the departments of Computational Biology and Bioinformatics.

\section{Gene-fusion analysis}

Fusion gene events were identified and classified through the use of CICERO, a local assembly-based algorithm that integrates RNA-seq read support with extensive annotation for candidate ranking. This data analysis was performed by the Center for Applied Bioinformatics Department and Computational Biology department at St. Jude Children's Research Hospital. Detailed methods for CICERO are as described [177]

\section{Analysis of apparent bivalency}

For bivalent gene promotor analysis using RNA-seq mouse tumor data we used reference datasets from [160] and [106] for direct comparisons. Statistical analysis of enrichment of upregulated bivalent targets in H3K27M gliomas, differential expression analysis was previously described [160]. 


\section{Whole Exome Sequencing Analysis}

Whole exome sequencing analysis was performed by the Center for Applied Bioinformatics Department and Computational Biology department at St. Jude Children's Research Hospital. Detail methods for exome-capture, illumina sequencing, identification of somatic mutations in mouse and mapping to human, and validation of somatic mutations to non-tumor mice can be found in the manuscript [178].

\section{Graphing and Statistical Analysis}

All graphs with statistical analysis were doing using Graph Pad Prism and software. All results were tested through an Ordinary one-way ANOVA and then individual comparisons were done by Tukey's multiple comparisons test. Survival plot analysis used log-rank Mantel-Cox test and Gehan-Breslow-Wilcoxon test. Statistical pvalues were as follows: ${ }^{*} \mathrm{p}<0.05,{ }^{* *} \mathrm{p}<0.01,{ }^{* * *} \mathrm{p}<0.001,{ }^{* * * *} \mathrm{p}<0.0001$.

\section{Fluorescence In Situ Hybridization}

The Cytogenetic Shared Resource Laboratory at St. Jude performed FISH. Ten formalin fixed embedded (FFPE) mouse brain tumor tissue specimens were submitted in order to determine by FISH if there is amplification of the FGFR2, EFGR, Met, PDGFR $\alpha$ genes in the tumors. Purified FGFR2 (RP23-158N2), EGFR (RP23-295E4), cMET (RP23-73G15), PDGRFa (RP23-55P22) were labeled individually with a reddUTP (AF594, Molecular Probes) and a chromosome 7 control probe (RP23-297D6/7A3) with a green-dUTP (AF488, Molecular Probes) by nick translation. Paraffin slides were deparaffinized with xylene. Pepsin was used for protease treatment. The labeled probes were hybridized to all the samples in a solution containing 50\% formamide, $10 \%$ dextran sulfate, and 2X SCC. The cells were then stained with 4, 6-diamidino-2-phenylindole (DAPI) and analyzed. 


\section{CHAPTER 3. RESULTS: DOUBLE STRAND BREAK REPAIR INACTIVATION LEADS TO HIGH-GRADE GLIOMAS}

A clear understanding of the role of DSBR during cortical development is crucial for piecing together the tissue-specific requirements for DNA repair throughout neurogenesis. Previous mouse models studies from our lab and others have helped to identify the importance of BRCA2 during HR and LIG4 in NHEJ during neural development. Three key findings from DNA repair deficient mouse models underpin the foundation and reasoning behind the genetic approach used in this chapter. First, germline inactivation of either Brca2 or Lig4 is lethal at mid or early gestation [179-181], although coincident inactivation of $p 53$ rescues lethality but promotes lymphoma and medulloblastoma formation $[49,58]$. Second, conditional neuraxis-wide inactivation of Brca2 or Lig4 via Nestin-cre, which expresses in neural progenitors throughout the brain [60], resulted in pronounced microcephaly. This microcephaly is associated with defects in neurogenesis embryonically and during postnatal cerebellar development $[49,58]$. Lastly, Brca2 $2^{\text {Nestin-cre }}$ or Lig4 $4^{\text {Nestin-cre }}$ in conjunction with $p 53$ inactivation restricted tumorigenesis to early onset medulloblastoma, which occur in the cerebellum or hindbrain $[49,58]$. Together these models have helped provide the framework and importance of BRCA2 (HR) and LIG4 (NHEJ) during nervous system development, especially to the granule neuron progenitor cell population in the external germinal layer of the developing cerebellum. However, much is still unknown about the roles these key DSBR factors play in cortical development. Therefore, work presented in this chapter further deciphers the basis for tissue-specific disruption of genomic stability and tumorigenesis in the mammalian cortex.

\section{Emx1-cre Deletion of DSBR, HR or NHEJ, Result in Cortical Cell Loss}

To prevent medulloblastoma formation and to determine how DSBR influences cortical development, Emxl-cre was used to target cortical progenitors. Emxl-cre expression occurs from embryonic day 9.5 (E9.5) primarily in the cortical progenitor cells of the dorsal telencephalon, which specifically develops into the cortex or forebrain. Comparatively, Nestin-cre, which was previously used, expresses in neural progenitors throughout the entire brain about a day later, between E10-E11 [60, 61, 73]. Conditional inactivation of Brca2 (Brca2 ${ }^{\text {LoxP/LoxP }}$; Emxl-cre hereafter called Brca2 $2^{\text {Emx I-cre; }}$;imilar nomenclature is used for additional mouse genotypes) was used to inhibit the HR pathway and inactivation of Lig4 ( $\left.\operatorname{Lig} 4^{\text {Emxl-cre }}\right)$ disabled NHEJ. Histological analysis to examine the cortex in 9-month-old mice revealed microcephaly in both DSBR-deficient mice (Figure 3-1A, B). Brca2 $2^{\text {Emxl-cre }}$ mice cortices were approximately $40 \%$ smaller compared to controls, whereas Lig ${ }^{\text {Emxl-cre }}$ cortices were about $85 \%$ smaller compared to control and $\sim 25 \%$ smaller than Brca2 $2^{\text {Emxl-cre }}$. This would suggest that both DSBR pathways are crucial for normal cortical development, with LIG4 (NHEJ) even more so than BRCA2 (HR). Because we predict that cellular loss is occurring during corticogenesis, we next looked at the rate of apoptosis during embryogenesis at E15.5 using activated Caspase-3, a surrogate marker for apoptosis, and TUNEL (data not 

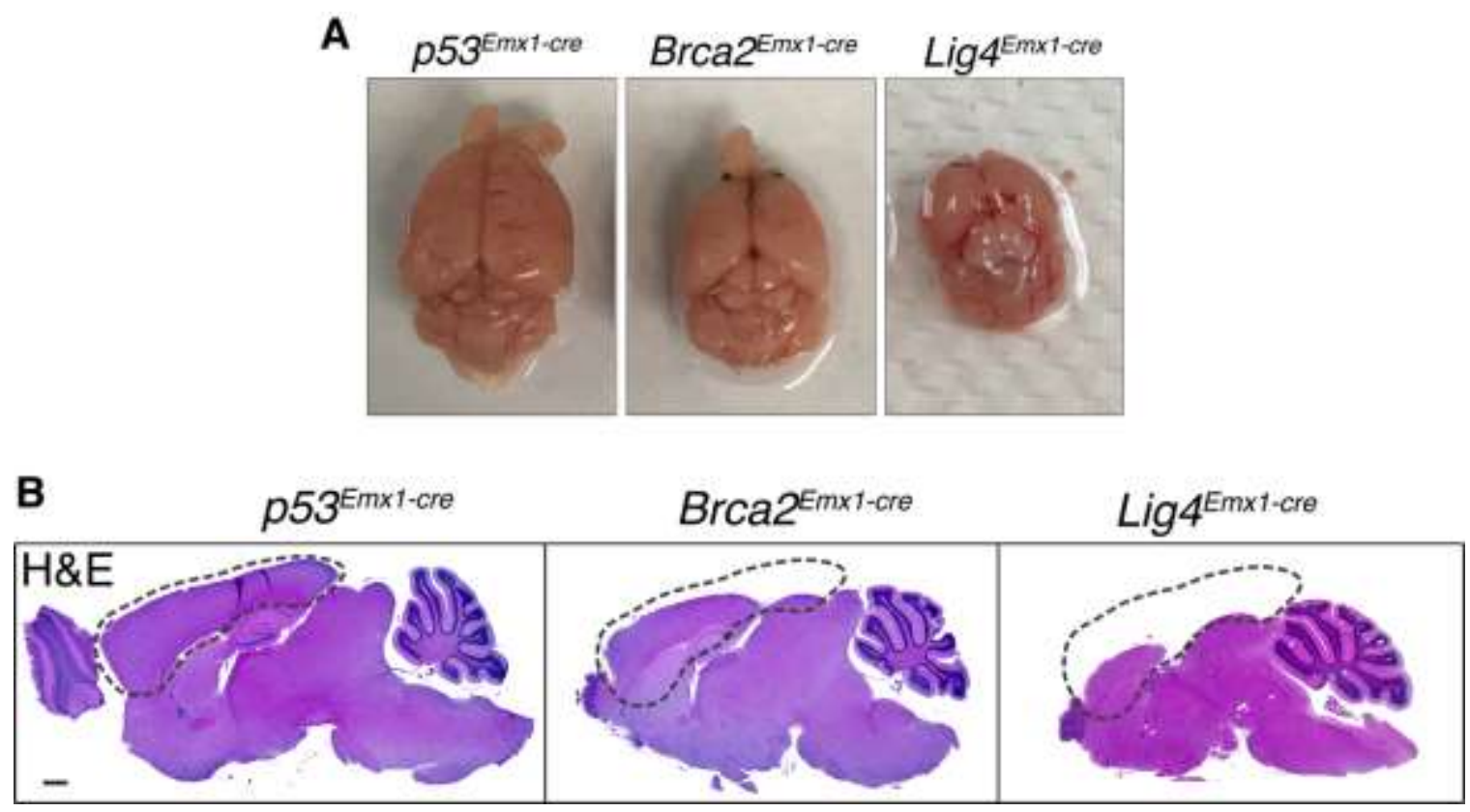

Figure 3-1. Emx1-cre inactivation of Brca2 or Lig4 result in cortical microcephaly.

(A) Mouse brains display reduced cortex size at 9 months of age in DSBR mutants. (B) H\&E staining of sagittal sections shows the difference in adult cortex size in DSBRdeficient mice compared to $p 53^{\text {Emxl-cre }}$ control. Brca2 $2^{\text {Emxl-cre }} 40 \%$ cortical reduction and Lig4 ${ }^{\text {Emxl-cre }} 85 \%$ reduction, highlighted by dotted line. Scale bar, $1 \mathrm{~mm}$. 
shown). Caspase-3 immunohistochemistry (IHC) staining revealed that loss of the respective repair factors impacted different cell populations and Lig4 inactivation resulted in extensive cell death compared to Brca2 mutant tissue (Figure 3-2A). Brca2 Emxl-cre mutants contained marginal levels of apoptosis in deep layers of the cortex compared to Lig4 $4^{\text {Emxl-cre }}$ where a majority of the cell death was localized in the upper layers aligning with non-proliferative progenitors. The marked increase and location of apoptotic cells in the developing cortex of the DSBR-deficient mutants is consistent with increased levels of DNA damage as evidenced via increased $\gamma \mathrm{H} 2 \mathrm{AX}$ expression (Figure 3-2A, B). $\gamma \mathrm{H} 2 \mathrm{AX}$-foci are strong indicators of DSBs, while pan-nuclear expression is indicative of cellular death $[51,182]$. Both foci and nuclear $\gamma \mathrm{H} 2 \mathrm{AX}$ expression were identified at E15.5 in lower and deep cortical layers of Lig4 ${ }^{\text {Emxl-cre }}$ embryos, although predominantly in the deep layers for Brca2 $2^{\text {Emxl-cre }}$. Overall, there was significantly more DNA damage found throughout the cortex in Lig4 ${ }^{\text {Emx I-cre }}$ mice than Brca $2^{\text {Emxl-cre (Figure }}$ 3-2B). E15.5 time period was initially selected as a point of interest because the second round of progenitor production begins and therefore is a highly proliferative stage of cortical development, as shown with PCNA, a proliferating cell marker. However, because the first round of cortical progenitors are produced at E12.5 we additionally investigated this time point. Similar to the results found at E15.5, Lig4 Emxl-cre embryos had significantly more $\gamma \mathrm{H} 2 \mathrm{AX}$ expression throughout the neocortex compared to $B r c a 2^{E m x l-c r e}$ at E12.5 (Figure 3-2C, D). Even three days earlier in embryogenesis it still appeared that Brca2 ${ }^{\text {EmxI-cre }}$ had more damage in the lower cortical layers. We also observed significant amount of caspase- 3 expression throughout the cortices of both

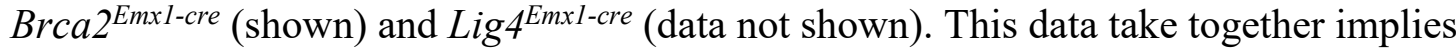
that while both DSBR pathways are critical in providing genome stability to the developing cortex they do so in a spatially preferential manner. BRCA2 and HR prevent DNA damage and cell death in the lower cortical cell layers while LIG4 and NHEJ provide a similar preventative mechanism throughout the entire cortex, however to a greater extent in the upper cell layers.

\section{DSBR-Deficiency and $p 53$ Inhibition in a Mouse Neocortex Results in Gliomagenesis}

Despite perturbed cortical development and increased DNA damage, the overall observable phenotype and survival of the DNA repair mutants was not obviously different to controls. Because we observed high levels of apoptosis in both Brca2- and Lig4-deficient mice neocortices we were interested in what the consequence would be to additionally inhibit p53-mediated apoptosis by crossing with a p53-conditional knock-out mouse. Furthermore, knowing that both (Brca2;p53) Nestin-cre and (Lig4;p53) Nestin-cre mice generate medulloblastomas from previous models our lab created $[49,58]$, we hypothesized that Emxl-cre would have a similar tumorigenic effect on the mouse cortex. We discovered, that coincident $p 53$ loss with Brca $2^{\text {Emxl-cre }}$ or Lig $4^{\text {Emxl-cre }}$ resulted in highgrade gliomas starting at 6 months of age (Figure 3-3A). Notably, inhibition of either repair pathway resulted in similar overall survival and tumor latency of about 10 months (Figure 3-3A). Interestingly, tumor incidence was about $35 \%(15 / 44)$ in $p 53^{\text {Emxl-cre }}$ mice, although this ultimately was after almost 21 months for these controls. Tumor incidence increased significantly to $44 \%(43 / 97)$ and $42 \%(45 / 108)$ in (Brca2;p53) ${ }^{\text {Emxl-cre }}$ and 
Figure 3-2. Emx1-cre inactivation of Brca2 or Lig4 result in DNA damage and cortical cell loss.

(A) Inactivation of Brca2 or Lig4 using Emx1-cre results in cellular apoptosis in distinct cortical layers at E15.5 marked by active Caspase-3 staining. High levels of DNA damage marked by $\gamma \mathrm{H} 2 \mathrm{AX}$ staining correlate with cellular loss. White hash mark indicates upper and deep cortical layers. Close ups; white arrow indicates $\gamma \mathrm{H} 2 \mathrm{AX}$-foci (DSBs), red arrow indicates pan-nuclear $\gamma \mathrm{H} 2 \mathrm{AX}$-signaling for cell death. PCNA is representative of high proliferation at this state and side box indicates cortical layers. (B) Quantification of E15.5 $\gamma \mathrm{H} 2 \mathrm{AX}$ expression (total foci and pan-nuclear) for upper and deep layers and total neocortex combined. $* * * * \mathrm{p}<0.0001$, n.s. $=$ not significant. $\mathrm{N} \geq 3$ for all embryo analysis. (C) Quantification of E12.5 $\mathrm{\gamma H} 2 \mathrm{AX}$ expression (total foci and pannuclear) for upper and deep layers and total neocortex combined. $* * * * p<0.0001$, $* * p=0.0011$. (D) DNA damage starts to accumulate at higher amounts during early embryogenesis at E12.5 in Lig4 ${ }^{\text {Emxl-cre }}$ mice more than Brca2 ${ }^{\text {Emxl-cre }}$. Representative image of apoptosis is shown in Brca2 $2^{\text {Emxl-cre }}$. All graphs represent mean values of replicates. Error bars indicate SEM. Abbreviations: VZ: ventricular zone, SVZ:

subventricular zone, IZ: intermediate zone, CP: cortical plate, MZ: marginal zone. 

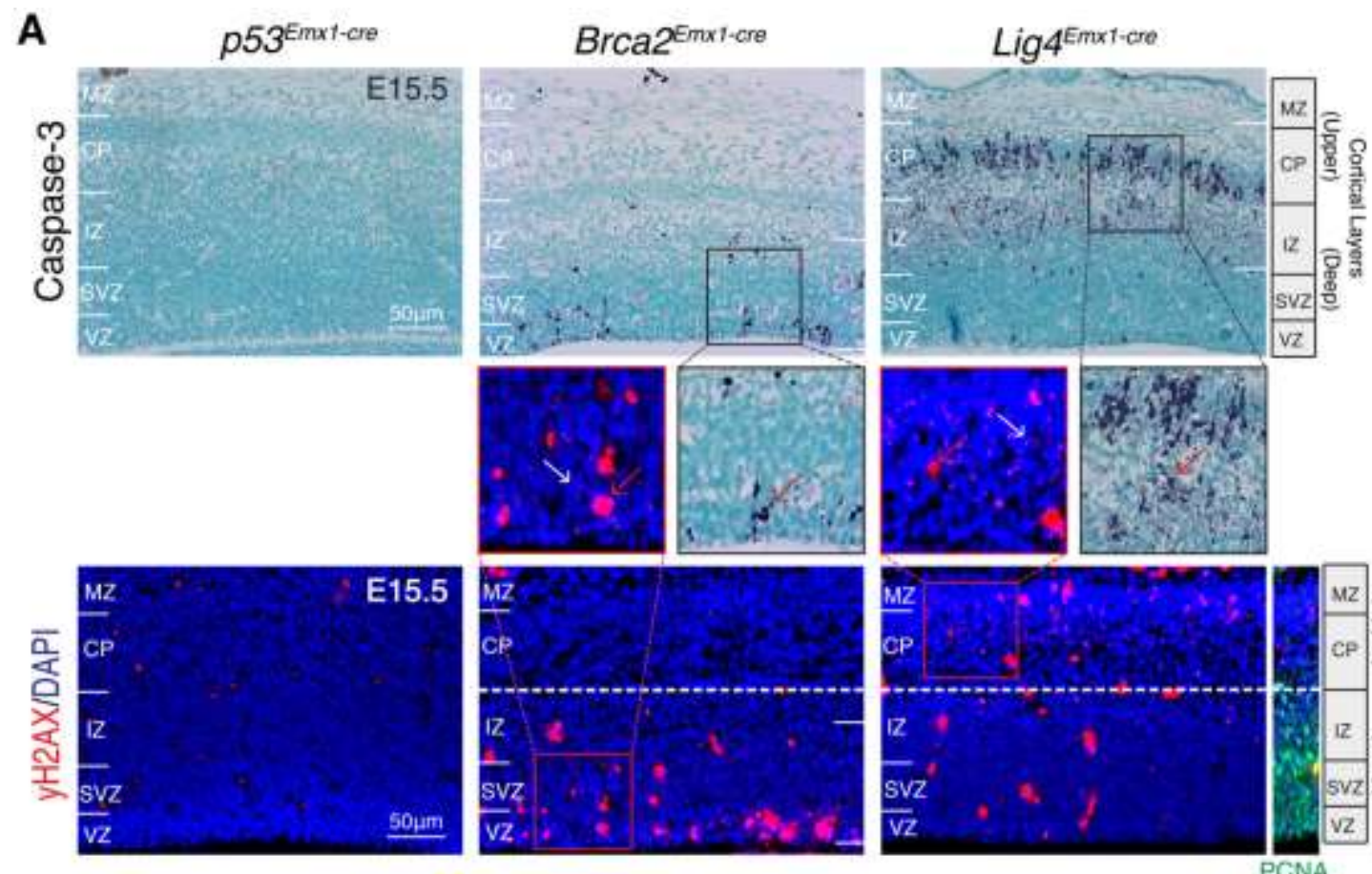

B

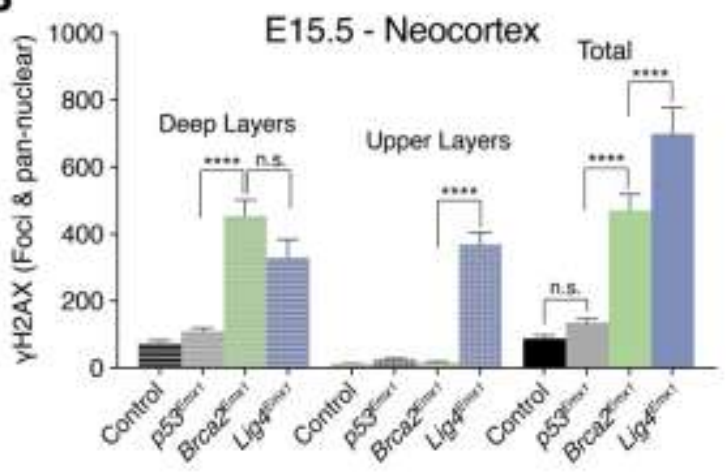

D

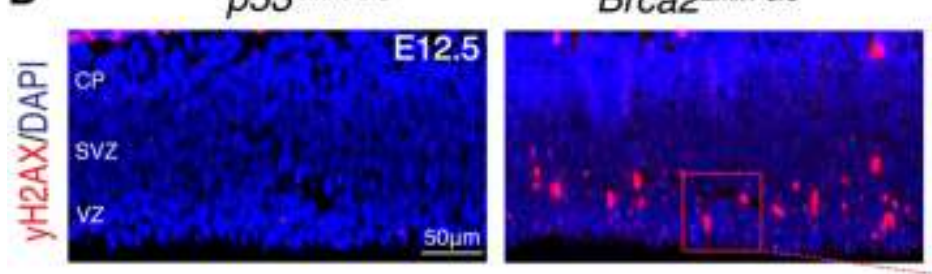

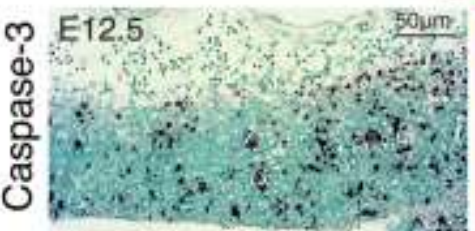

Brca2 eimutice

C
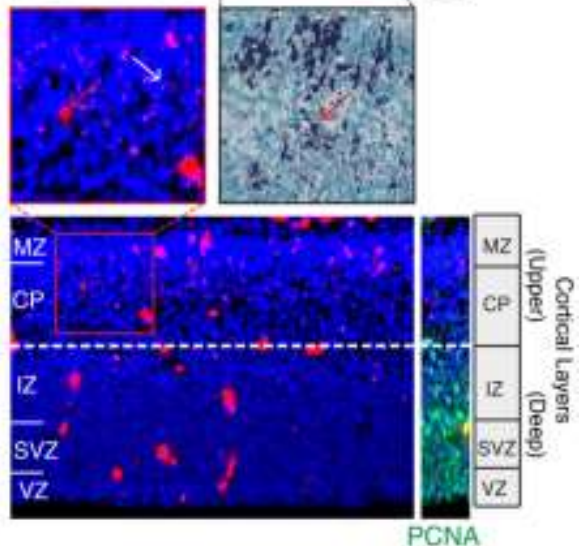

E12.5 - Neocortex

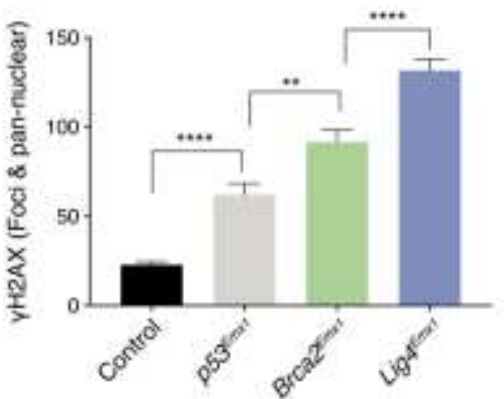

Lig4 $4^{\text {Emxt-cre }}$

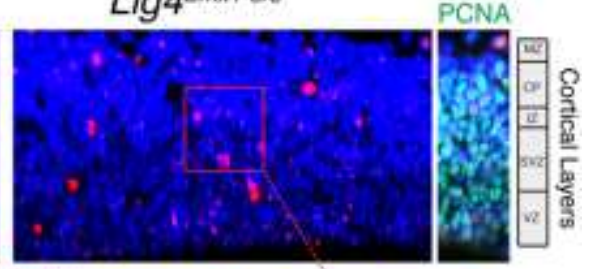

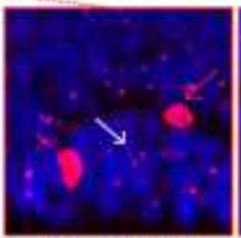

Brca2 Emrtere

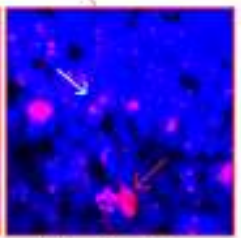

$\operatorname{Lig} 4^{\text {Enxatere }}$ 
Figure 3-3. DSBR-deficiency and p53 inactivation in a mouse neocortex results in gliomagenesis.

(A) Kaplan-Meier survival analysis in mice with induced mutant $p 53^{\text {Emxl-cre }}(\mathrm{n}=15 / 44)$, (Brca2;p53) Emxl-cre $(\mathrm{n}=43 / 97)$, or (Lig4;p53) Emxl-cre $(\mathrm{n}=45 / 108)$; Tumor incidence: $(\mathrm{n}=$ \# mice died of glioma / \# total mice), ${ }^{* * * *} \mathrm{p}<0.0001$. n.s., not significant; $\mathrm{p}=0.8869$.

(B) Representative images of gliomas (top panels). Sagittal sections stained for H\&E. IHC of PCNA, Olig2 and GFAP of representative HGGs display differences in glial cell populations. White dashed lines outline primary tumor(s). PCNA indicates tumor cell proliferation and diffusion; numbers $(4,4 \& 5)$ denote diffusion score in panel C. Scale bar, $1 \mathrm{~mm}$ for whole brain sections; $200 \mu \mathrm{m}$ for higher-magnification images. (C) Glioma diffusion scores based on H\&E and PCNA expression. Least diffuse (1) to highest (5). Percentage of tumors analyzed with determined diffusion score. Representative tumors for scores shown in panel B. p53 $3^{\text {Emxl-cre }}(\mathrm{n}=4),(\operatorname{Brca} 2 ; p 53)^{\text {Emxl-cre }}(\mathrm{n}=16)$, or (Lig4;p53) Emxl-cre $(\mathrm{n}=9)$ (D) Mouse characteristic glioma histopathology of pHGG classifications with H\&E. HGGs vary in classification of cell type involved in tumor formation. (Analysis done by Dr. David Ellison). Scale bars: $100 \mu \mathrm{m}$ (Lowermagnification images), $50 \mu \mathrm{m}$ (Higher-magnification images). 

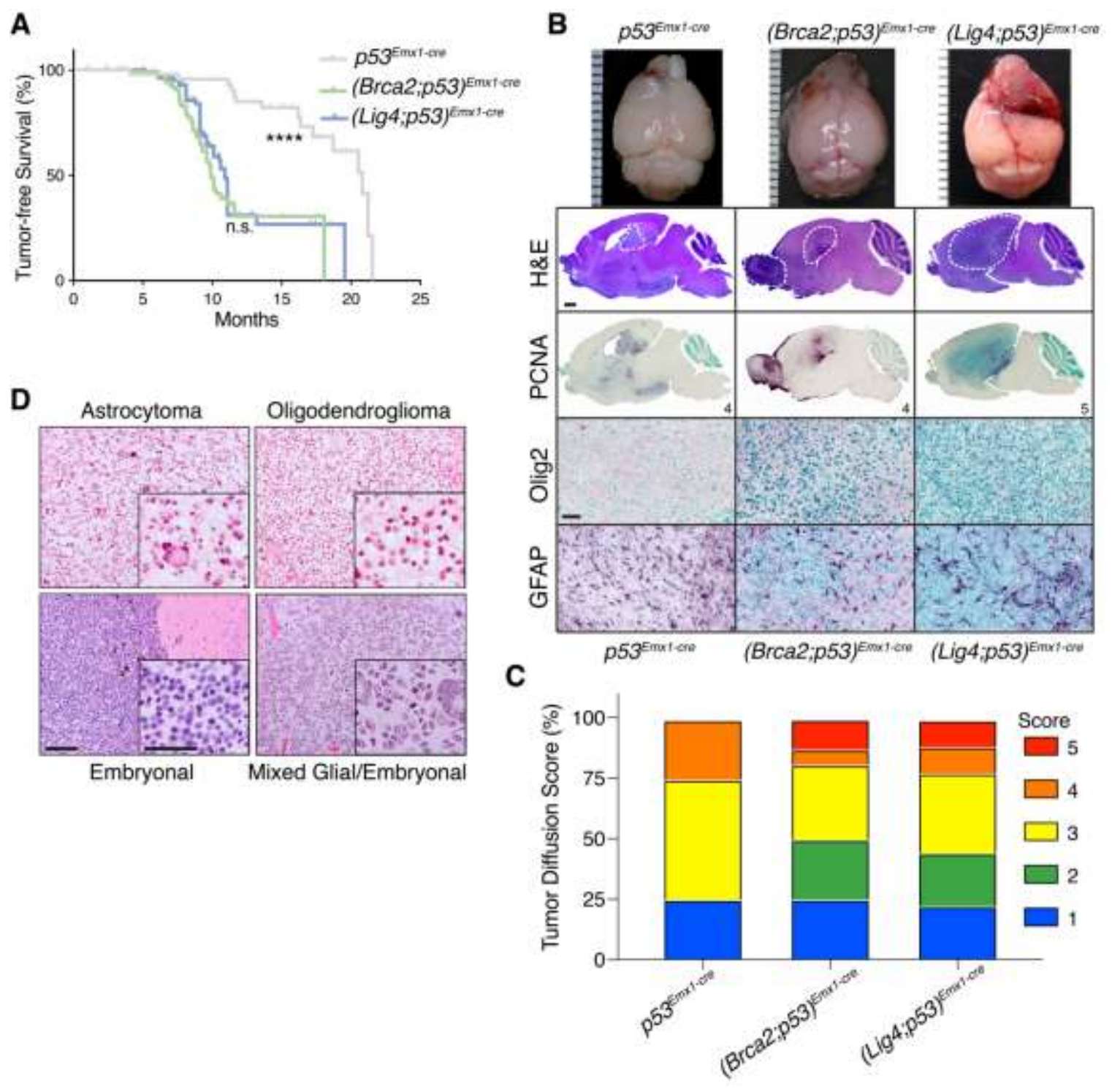
(Lig4;p53) ${ }^{\text {Emxl-cre }}$, respectively. Both DSBR-deficient gliomas display similar focal tumor patterns throughout the cortices and olfactory bulbs with moderately low levels of diffusion as seen in H\&E and PCNA expression, which is commonly used to detect tumor cells as they usually undergo high rates of proliferation (Figure 3-3B). These markers were used to apply an unbiased diffusion score (1-5) based on localization of primary tumor and overall dispersal of cancerous cells in representative samples of mouse gliomas, as shown in (Figure 3-3B, C). Both DSBR mutant tumors exhibited increased nuclear Olig2 expression (oligodendrocytic marker) and variable expression of GFAP (marker for astrocytes and ependymal differentiated cells) compared to $p 53^{\text {Emxl-cre }}$ mouse tumors (Figure 3-3B). Similar pathology is often identified in pHGGs, in addition to moderate nuclear pleomorphism (H\&E), increased mitotic activity (PCNA) and broad infiltration, all of which we identified [92, 137]. A subset of mouse gliomas were assessed and classified by Dr. David Ellison, a clinical neuropathologist. The resultant mouse gliomas were mostly heterogeneous, displaying classical features of several different subtypes of HGGs, including astrocytomas, oligodendrogliomas, embryonal and mixed glial gliomas (Figure 3-2C). To note, $p 53^{E m x}$ l-cre mice eventually succumbed to HGGs, albeit much later in age and at far lower frequencies (Figure 3-2A, B). Although, this was not a surprise as p53 is not only a key protein in the apoptotic pathway but is also one of the most frequently mutated tumor suppressors in all types of cancer, including brain tumors $[82,88,90,183]$. These data indicate disruption of genome integrity by inhibition of $\mathrm{Brca} 2$ (HR) or Lig4 (NHEJ) coupled with $p 53$ inactivation in cortical progenitors strongly predisposes mice to HGGs.

\section{Distinct Gene Expression Profiles Characterize HGG Arising from Disabled DSBR}

HGGs typically display heterogeneity within subgroup classifications defined by gene expression signatures including proneural, classical or proliferative and mesenchymal $[121,125,160,184]$. To explain potential mechanisms resulting in heterogeneous glioma formation and diffusion from Brca2-or Lig4-deficiency in the mouse cortex, we collected tumor tissue from (Brca2;p53) $)^{\text {Emxl-cre }},\left(\right.$ Lig4;p53) Emxl-cre $^{\text {and }}$ p53 $3^{\text {EmxI-cre }}$ mutants and performed transcriptome analysis through RNA-sequencing (RNA-seq). Unsupervised clustering of HGGs revealed unique grouping of the most differentially expressed genes between (Brca2;p53) ${ }^{\text {Emxl-cre }}$ and (Lig4;p53) ${ }^{\text {Emx I-cre }}$ gliomas, reflective of partial tumor heterogeneity (Figure 3-4A). We identified 243 uniquely upregulated genes in (Brca2;p53) EmxI-cre samples and 32 in (Lig4;p53) EmxI-cre using standardized expression cutoffs. Initial glance at the 32 genes upregulated in (Lig4;p53) ${ }^{\text {Emxl-cre }}$ tumors we found three were Sox genes $(\operatorname{Sox} 3,10,13)$, all involved in embryonic development, cell fate determination and neural precursor differentiation . In addition to Sox 10, Insulin like growth factor 2 (IGF-2), was upregulated and interestingly both of these genes are identified as enhancers of PDGFR $\alpha / \beta$ in pediatric gliomas, specifically oligodendrogliomas (Sox10) [185-188]. Lastly, the proto-oncogene B-cell CLL/lymphoma 3 (BCL3) was upregulated in (Lig4;p53) Emxl-cre tumors, which recently has been associated with resistance to chemotherapy in gliomas [189]. Gene-set enrichment analysis (GSEA) was performed using Enrichr, which is used for evaluating differentially expressed gene targets associated with pathways. Through Enrichr, we 
Figure 3-4. Gene expression profiles characterize HGGs arising from disabled DSBR.

(A) Unsupervised clustering of $(\operatorname{Brca2} ; \text {; 53) })^{\text {EmxI-cre }}(\mathrm{n}=16)$ and $(\text { Lig4;p53) })^{\text {Emxl-cre }}(\mathrm{n}=$ 10) HGGs reveal unique groupings of most differentially expressed genes reflective of partial tumor heterogeneity. 243 genes upregulated in (Brca2;p53) Emxl-cre and 181 genes

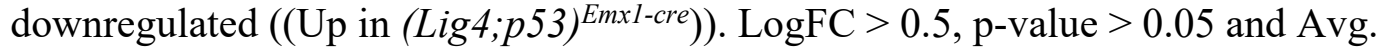
Expression $>1.0$. (B) Enrichr analysis indicates significantly enhanced associated pathways in (Brca2;p53) Emxl-cre or (Lig4;p53) Emxl-cre tumor samples when compared to each other. (C) Ccnd3 and Map3kl display higher amplification in (Lig4;p53) EmxI-cre. (Fragments Per Kilobase of transcript per Million mapped reads). (D) Enrichr pathway analysis of DSBR-deficient gliomas juxtaposed to control $p 53^{\text {Emxl-cre }}(\mathrm{n}=6)$ identifies upregulation of genes involved in DNA damage response and RNA processes, in

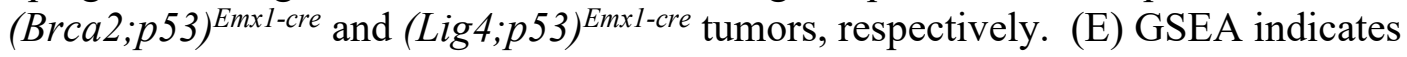
DSBR-deficient tumors are less astroglial and oligodendrocytic in cell signatures compared to $p 53^{E m x l-c r e}$ tumors alone. Cahoy dataset was used for glial cell signature analysis. (F) Comparing (Brca2;p53) Emxl-cre $^{\text {to }}\left(\right.$ Lig4;p53) ${ }^{\text {Emxl-cre }}$ identifies Brca2 as more neuronal and Lig4 more oligodendrocytic. 

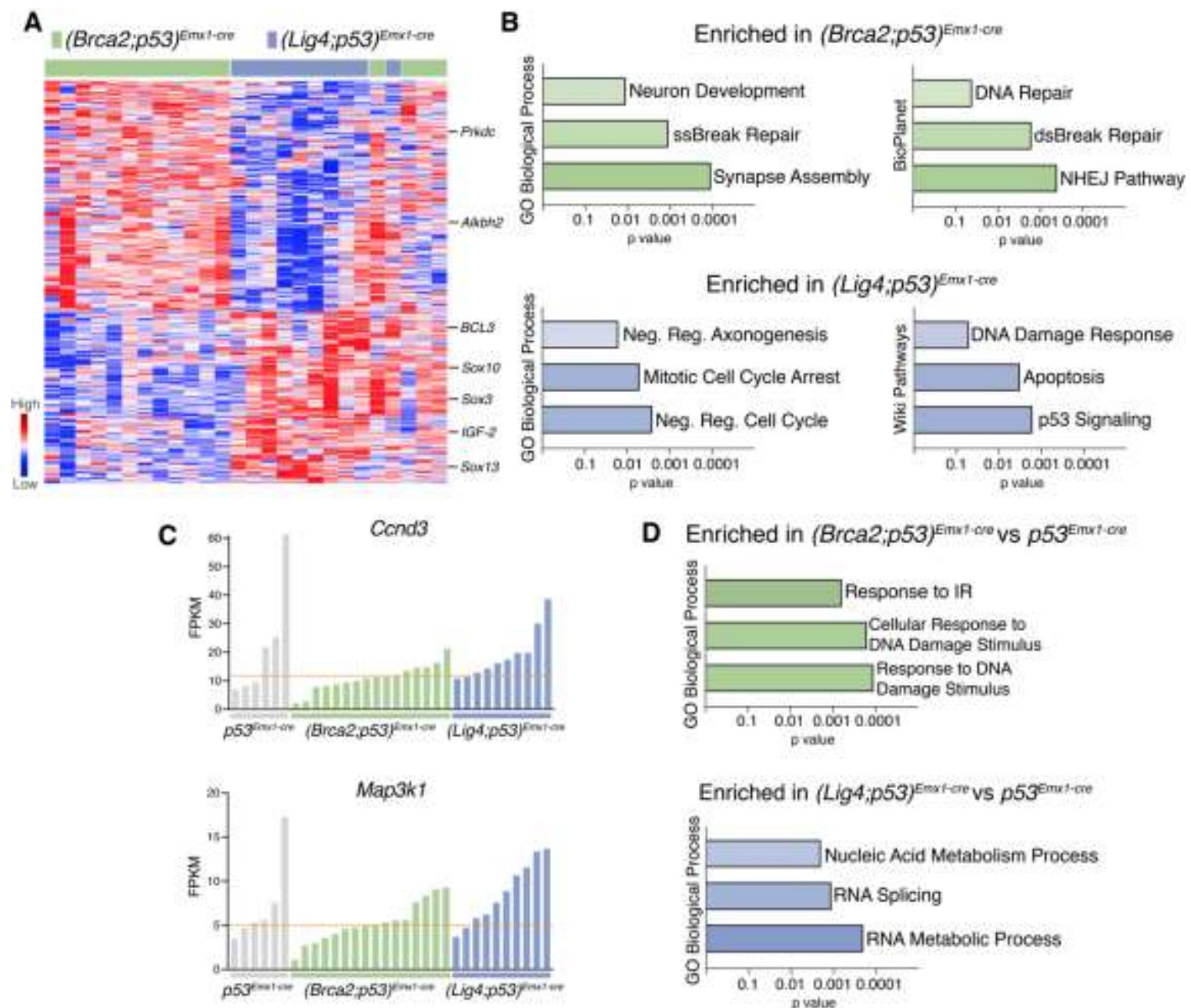

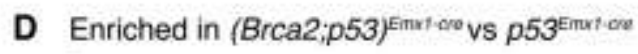

E

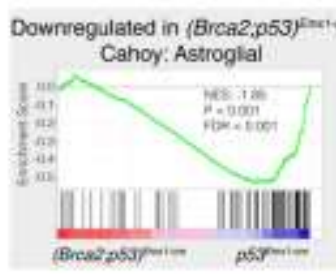

Downregulated in (Lig4, p53) Foive

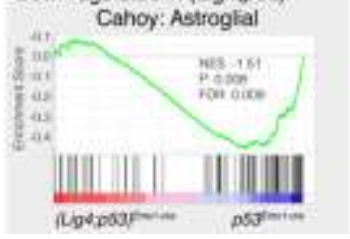

Downregulated in (Brca2 p5s) fmen-m

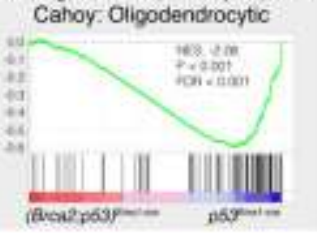

Downregulated in (Lig4 p5:37 Cahoy: Oligodendrocytic

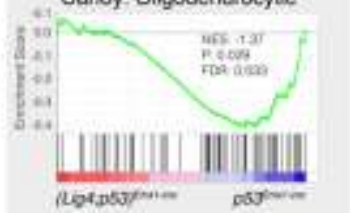

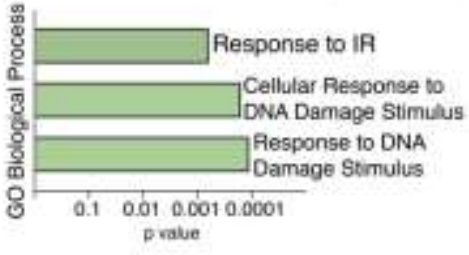

Enriched in ( Lig4;p53) Emertare vs p53 Eimut-me

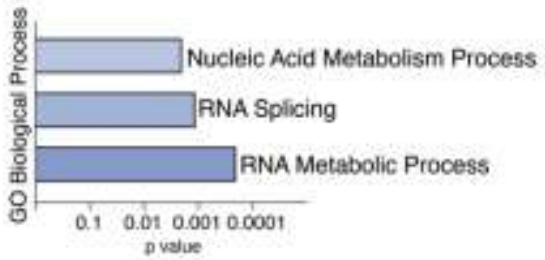

F
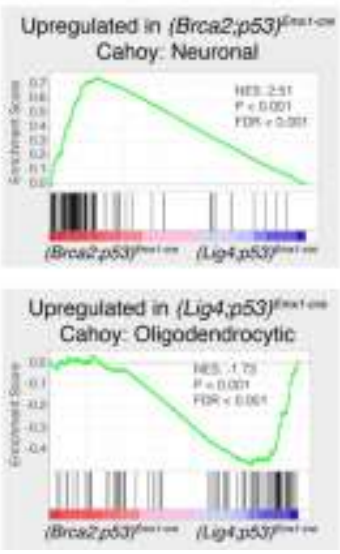
identified associated pathways involving neuron development, synapse assembly and compensatory DNA repair mechanisms significantly upregulated in (Brca2;p53) Emxl-cre mice (Figure 3-4B). Notable genes enriched in the (Brca2;p53) ${ }^{E m x 1-c r e ~ g l i o m a s ~ w e r e ~}$ tyrosyl-DNA phosphodiesterase 1 ( $T d p 1)$, protein kinase, DNA-activated, catalytic subunit $(P r k d c)$, alkB homolog 2 and alpha-ketoglutarate dependent dioxygenase (Alkbh2). These genes are all directly involved in repairing various forms of DNA damage, including single and double strand breaks that prevent neurodegenerative diseases or cancers [190-192]. In Lig4-deficient samples we observed enrichment of pathways encompassing regulation of axonogenesis, cell cycle and other DNA damage response factors (Figure 3-4B). Cyclin D3 (CCND3) and mitogen-activated protein kinase kinase kinase $1(M A P 3 K 1)$ genes were more upregulated in the (Lig4;p53) Emx1-cre tumors than (Brca2;p53) ${ }^{\text {Emxl-cre }}$ (Figure 3-4C). Alterations in CCND3 and MAP3KI kinases are of particular interest because they have been previously correlated with pediatric brain tumor development and tumor progression [103, 134, 178, 193]. A direct comparison of (Brca2;p53) Emxl-cre and p53 $3^{\text {Emxl-cre }}$ tumors through Gene Ontology, which analyzes gene functions through an enrichment analysis database, revealed additional DDR pathway genes enriched, specifically in the $\mathrm{Brca}$-deficient groups:

BRCA1/BRCA2-containing complex subunit 3 ( $B r c c 3$ ), Blm RecQ like helicase or Blm, RAD51 and Rad18, structural maintenance of chromosomes 1A (SMC1A), and the chromatin remodeler $A T R X$ (Figure 3-4D). Other genes related to regulation of $p 53$ and apoptosis were upregulated in (Brca2;p53) ${ }^{\text {Emx } 1-c r e}$ gliomas compensating for loss of $p 53$, which include; apoptosis enhancing nuclease $(A E N)$, BTG anti-proliferation factor 2 (BTG2), and apoptosis inducing factor mitochondria associated 1(AIFM1) (Figure 3-4D). These results again highlight how glioma cells gene regulation may be attempting to compensate for the loss of functions induced by mutations in DSBR and $\mathrm{p} 53$-mediated apoptosis. Conversely, enriched pathways in (Lig4;p53) Emxl-cre compared to $p 53^{\text {Emxl-cre }}$ tumors involved RNA related processes. Within the RNA effected pathways the RNA Binding Motif Protein X-Linked $(R B M X)$ gene was identified. $R B M X$ was discovered in a genome-wide siRNA screen and was found to be directly associated with $B R C A 2$ and HR repair [194]. Significantly enriched genes and associated pathways in DSBR gliomas possibly reveal the reliance of tumor cells on compensatory DNA repair pathways to survive and proliferate.

Determining the cellular origin for pediatric brain cancers is currently the focus of extensive research, and in most cases the actual tumor cell of origin remains enigmatic [195]. It's unclear if brain tumors originate from stem cell-like populations or from specific progenitor cell subtypes such as neurons, astrocytes or oligodendrocytes [97]. To examine the cell of origin in gliomas generated by loss of Brca2 or Lig4 we utilized a specific transcriptome database developed by a group led by Ben A. Barres (Cahoy) for specific neural cell types using GSEA [196]. GSEA revealed that DSBR mutant gliomas displayed varied maturity cellular states of origin with astroglial and oligodendrocytic cellular populations significantly decreased when compared to control $p 53^{\text {Emxl-cre }}$ tumors (Figure 3-4E). Astroglial expression patterns are defined as cultures derived from small unidentified populations of proliferating glial precursor cells that express several different astrocyte markers, but appear to have an immature phenotype [196]. In contrast, when (Brca2;p53) ${ }^{\text {Emxl-cre }}$ gliomas were compared to (Lig4;p53) Emxl-cre, Brca2 cKO showed a 
neuronal signature $(\mathrm{p}<0.001)$ while Lig4 $\mathrm{cKO}$ tended to be oligodendrocytic $(\mathrm{p}<0.001)$ (Figure 3-4F). Histology supports similar heterogenous cell populations effected within DSBR-deficient mutants, especially increased Olig2 (oligodendrocyte) expression in (Lig4;p53) Emxl-cre tumors (Figure 3-2B). Thus, Brca 2 and Lig4 cKO gliomas have distinct differences in gene expression patterns with enrichment of unique gene sets for specific brain cell populations, which may reflect the differential requirements during neural development [14]. Gene expression analysis suggests that glioma cells with loss of Brca2 (HR) result in significantly upregulating genes to respond to DNA repair deficiency, while loss of Lig4 (NHEJ) seem to be more concerned with upregulating genes to increase tumorigenesis.

\section{Recurrent Chromosomal Rearrangements Characterize DSBR-Deficient HGGs}

Chromosomal rearrangements resulting in gene-fusions and DNA copy number aberrations can lead to gene amplifications which are all common occurrences in many types of human cancers, including gliomas [90, 141, 177]. Because faulty rearrangements are due to improperly repaired DSBs [197, 198], we predicted that loss of HR, which is less error prone, would result in more chromosomal alterations in gliomas than NHEJdeficiency. To identify gene-fusions we utilized a new algorithm developed by the St. Jude Computational Biology department, called CICERO, that combines RNA-seq with extensive annotation for gene candidate rankings which help identify driver fusions better than the traditional canonical exon-to-exon chimeric transcripts [177]. Some of the most frequent oncogenic fusion events or gene amplifications found in HGGs include MET, PDGFR , BRAF, SOX6, PTEN and KRAS (details are discussed as identified below) [80, $90,199]$. Utilizing both transcriptome and whole exome sequencing from mouse tumor samples we identified various potential gene-fusions and amplifications with unique structural variants. One of the most common identified fusions, particularly in more than $30 \%$ of analyzed (Brca2; 53$)^{\text {Emx l-cre }}$ tumors, was Met, which encodes the protein c-MET, a known tyrosine kinase (Figure 3-5A). The Met fusion partner was most frequently Capza2. MET fusions are also found in about $5 \%$ of pediatric glioblastoma multiforme and $10 \%$ of pHGG cases [199]. In addition, we found higher Met and Pdgfra amplifications in the mouse samples when compared to a human glioma dataset (Figure 3-5A, B). Chromosomal abnormalities of Met and Pdgfr $\alpha$ were confirmed using fluorescence in situ hybridization (FISH) and IHC (Figure 3-5B). Other gene alterations identified that have been extensively documented to have direct involvement of neurodevelopment and tumorigenesis were Braf, Sox6, Kras and Pten (Figure 3-5C, D) $[101,124,132,200-202]$. BRAF mutations are found in about $10 \%$ of pediatric cortical HGGs and more frequently in rare cancers like pleomorphic xanthoastrocytomas and anaplastic gangliomas [80, 88]. The specific $B R A F$ V600E mutation, which encodes a Ras-regulated serine/threonine kinase, was one of the only activating mutations found in a p53 $3^{\text {Emxl-cre }}$ glioma [201] (Figure 3-5C). Colorimetric IHC analysis and comparison to human glioma data confirmed the uniqueness of this specific mutation within the sample set (Figure 3-5C). We also identified overexpression of Sox6 in a many of DSBR deficient tumors. Sox6 is a transcription factor important for neurodevelopment and its amplification has been found in human glioma patients [202]. Additionally, the potent 
Figure 3-5. Recurrent chromosomal rearrangements characterize DSBR-deficient HGGs.

(A) Met gene-fusions were the most common fusion event identified in mouse tumor samples, particularly in the (Brca2;p53) ${ }^{\text {Emxl-cre }}$ group. FISH confirmed amplification of MET in mouse gliomas. Compared to human glioma samples, mouse tumors had on average higher overall expression values (FPKM - Fragments Per Kilobase of transcript per Million mapped reads). (B) Pdgfr $\alpha$ is overexpressed in many mouse HGGs. IHC and FISH analysis confirm PDGFR $\alpha$ over-expression and amplification, respectively. Pdgfr $\alpha$ was also more highly expressed in mouse tumor samples than human examples. Scale bar, $1 \mathrm{~mm}$ for whole brain sections, $100 \mu \mathrm{m}$ for higher-magnification images. (C) Structural variants of Braf were found in all tumor groups, including a ZEB2-BRAF fusion in a (Lig4;p53) ${ }^{\text {Emxl-cre }}$ sample. IHC staining identified Braf point mutation V637E (V600E - human) in a $p 53^{\text {Emx l-cre }}$ tumor. This mutation was also identified in three human samples from a previous database. Scale bar, $1 \mathrm{~mm}$ for whole brain sections, $100 \mu \mathrm{m}$ for higher-magnification images. (D) Sox6, Kras, and Pten are commonly altered genes in pHGGs and are overexpressed in select mouse gliomas. (E) (Brca2;p53) Emx1-cre $(\mathrm{n}=7)$ tumors had more overall somatic mutations on average than (Lig4;p53) ${ }^{\text {Emxl-cre }}(\mathrm{n}=$ 8 ) tumors from whole exome sequencing $(>5$ reads per mutant and variant allele frequency $>0.1, \mathrm{p}$-Value $=0.0006$ ). 


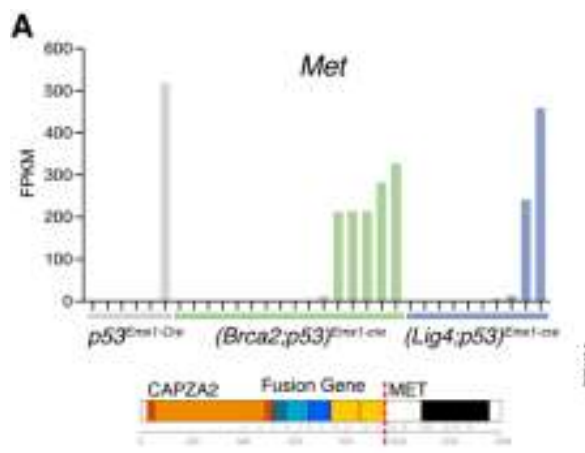

B
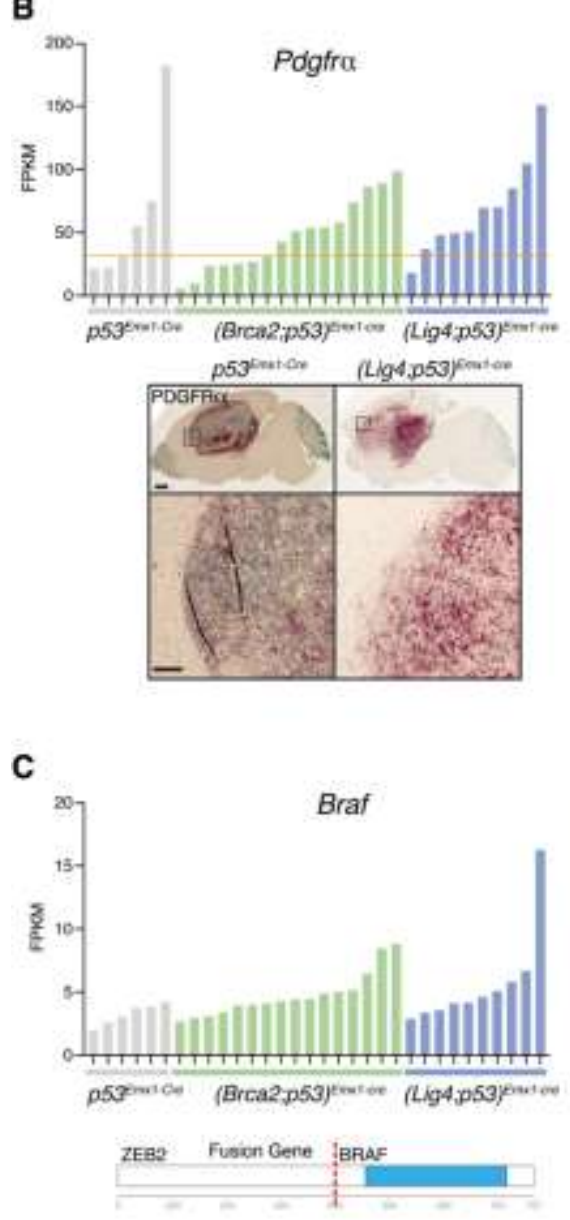
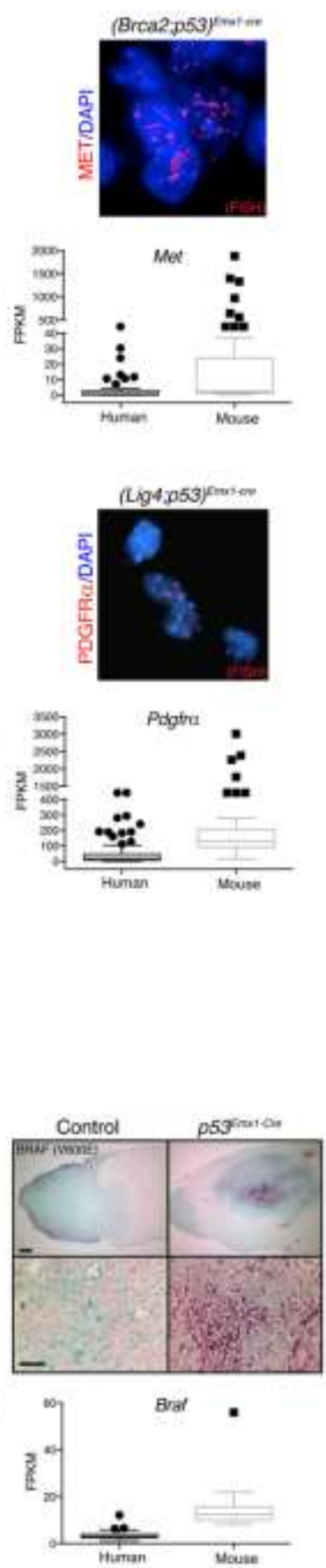

D
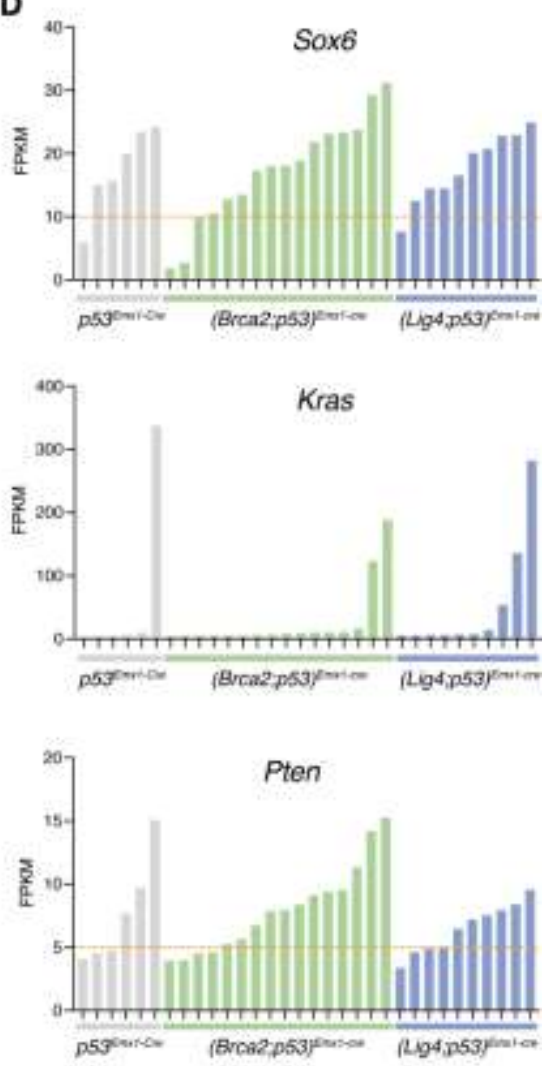

E

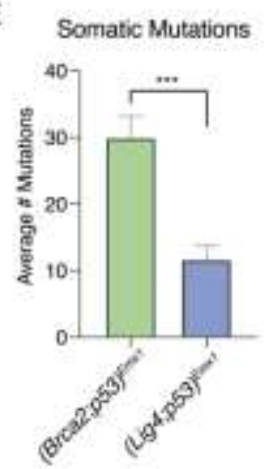


oncogene Kras, was overexpressed in a few of the HGG samples, implicating its role in assisting in tumorigenesis. Interestingly, Pten, a common tumor suppressor, had increased expression in about $2 / 3$ rds of the glioma samples, which is unexpected for a tumor suppressor, however overexpression of other tumor suppressors have been identified in some brain tumors [203]. Lastly, from whole exome sequencing we found the average number of somatic mutations (single-nucleotide variations) in the (Brca2;p53) Emx1-cre tumors ( 32) was almost three times that of the (Lig4;p53) ${ }^{\text {Emxl-cre }}$ mice $(\sim 12)$ (Figure 3-5E). These data together suggest loss of HR results in accumulation of more mutations, including gene-fusion alterations known to cause HGGs. This is likely due to cortical progenitors having to rely primarily on the more error-prone form of DSBR, NHEJ, during the earliest and most critical time period of development.

Inhibition of DSBR pathways through inactivation of Brca2 (HR) or Lig4 (NHEJ) with concurrent inhibition of p53 in the mouse cortex resulted in HGG formation. Analysis of tumor histology determined glioma classifications and diffusion based on abnormal cellular morphology including glial specific populations. Further investigation into tumor gene expression profiles revealed differences in gene dysregulation between HR- and NHEJ-deficiency. Based on the correlation with DSBR-deficiency and glioma development, we explored the possible cause of this tumorigenesis by examining genomic integrity during mouse embryogenesis.

\section{DSBR-Deficiency and p53 Inactivation in Neocortex Result in Selective DNA Damage Accumulation}

To determine if BrCa2- or Lig4-deficiency during early cortical development caused DNA damage, we examined $\gamma \mathrm{H} 2 \mathrm{AX}$ in (Brca2;p53) Emx1-cre $^{\text {and }}($ Lig4;p53) Emx1-cre cortices at different stages of development. During early development at E12.5 and E15.5, DNA damage accumulation, shown by $\gamma \mathrm{H} 2 \mathrm{AX}$-foci, was quantified in the neocortex (Figure 3-6A-D). The (Brca2;p53) Emxl-cre mutant mouse had slightly higher DNA damage at E12.5 than (Lig4;p53) Emxl-cre (Figure 3-6A, B). Even at this early period of development a subtle difference can be noticed between the location of $\gamma \mathrm{H} 2 \mathrm{AX}$-foci, with (Brca2;p53)Emxl-cre damage being in the lower SVZ and VZ layers and (Lig4;p53) ${ }^{\text {Emxl-cre }}$ having more damage in the upper MZ, CP and IZ layers. However, the difference in spatial DNA damage becomes much clearer three days later in corticogenesis. At E15.5, (Brca2;p53) ${ }^{\text {Emxl-cre }} \gamma \mathrm{H} 2 \mathrm{AX}$-foci were noticeably condensed in the lower or deep cortical layers corresponding to the highly proliferative progenitors marked by PCNA, suggesting that DSBs resulting from Brca2-deficiency occurred during replication (Figure 3-6C, D). Conversely, in the (Lig4;p53) Emx1-cre brain at E15.5, the greatest number of foci were identified and concentrated in the upper most differentiated cortical cell layers (cortical plate and marginal zone), although marginal amounts of damage were also found throughout other cortical layers (Figure 3-6C-D). The difference in the primary location of DNA damage within the cortices of Brca2 and Lig4 mutant mice is likely due to the specific repair pathway available during particular stages of cellular development. HR is the primary repair pathway used by the earliest 
Figure 3-6. DSBR-deficiency and p53 inactivation in neocortex result in selective DNA damage accumulation.

(A) DNA damage, in the form of $\gamma \mathrm{H} 2 \mathrm{AX}$-foci, accumulates more in (Brca2;p53) Emx1-cre during early embryogenesis at E12.5 than (Lig4;p53) ${ }^{\text {Emx } 1 \text {-cre }}$ in the neocortex. PCNA is representative of high proliferation throughout cortex at this state and side box indicates cortical layers. Dashed white line indicates approximate division of upper and deep cortical layers. Red box closeups highlight $\gamma \mathrm{H} 2 \mathrm{AX}$-foci in cortical layers. (B) Total number of $\gamma \mathrm{H} 2 \mathrm{AX}$-foci quantified in $150 \mu \mathrm{m}^{2}$ area. $\mathrm{N} \geq 3$ animals per groups, $\geq 9$ images per group. $* * * * p<0.0001, * p=0.0265$. (C) 3 days later in embryonic development at E15.5 the amount of DNA damage accumulation separates into cortical layered differences by DSBR mutation. (Lig4;p53) Emx1-cre mice have significantly higher levels of damage in upper layers, while (Brca2;p53) Emxl-cre mice have more damage in the deep neocortex. Dashed white line indicates division of upper and deep cortical layers. Red box closeups highlight $\gamma \mathrm{H} 2 \mathrm{AX}$-foci in cortical layers. Pan-nuclear $\gamma \mathrm{H} 2 \mathrm{AX}$ stain may be indicative of cell death or non-specific background. (D) Number of $\gamma \mathrm{H} 2 \mathrm{AX}$ foci quantified for upper and deep layers and total neocortex combined. Deep layers: $330 \mu^{2}$ area quantified; Upper layers: $200 \mu \mathrm{m}^{2}$ area quantified; Total all layers: $550 \mu \mathrm{m}^{2}$ area quantified. $\mathrm{N} \geq 3$ animals per groups, $\geq 9$ images per group. $* * * * p<0.0001, * p=0.0495$. All graphs represent mean values of replicates. Error bars indicate SEM. Abbreviations: VZ: ventricular zone, SVZ: subventricular zone, IZ: intermediate zone, CP: cortical plate, MZ: marginal zone. 

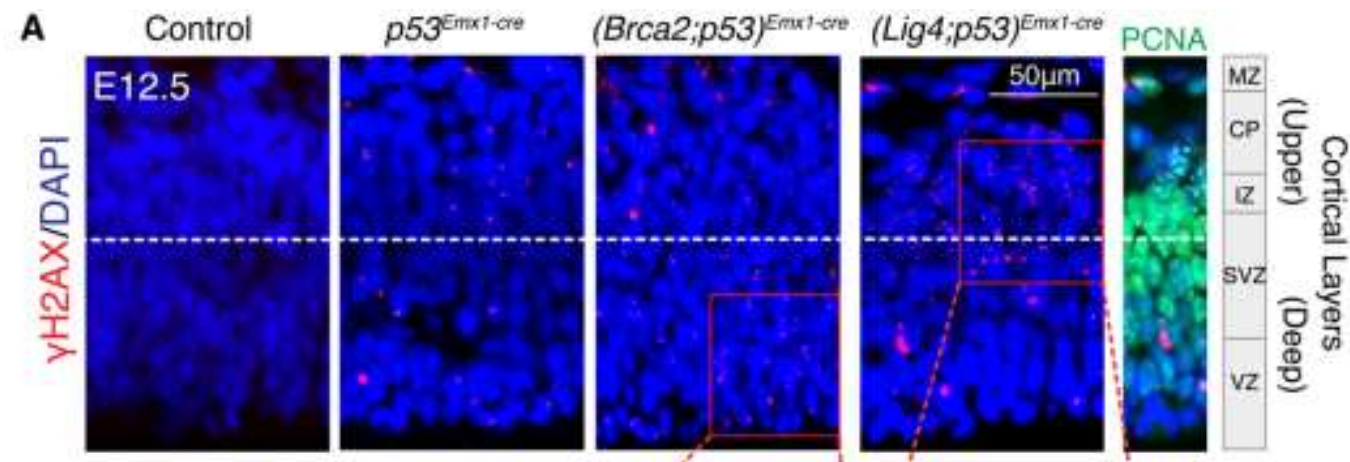

B
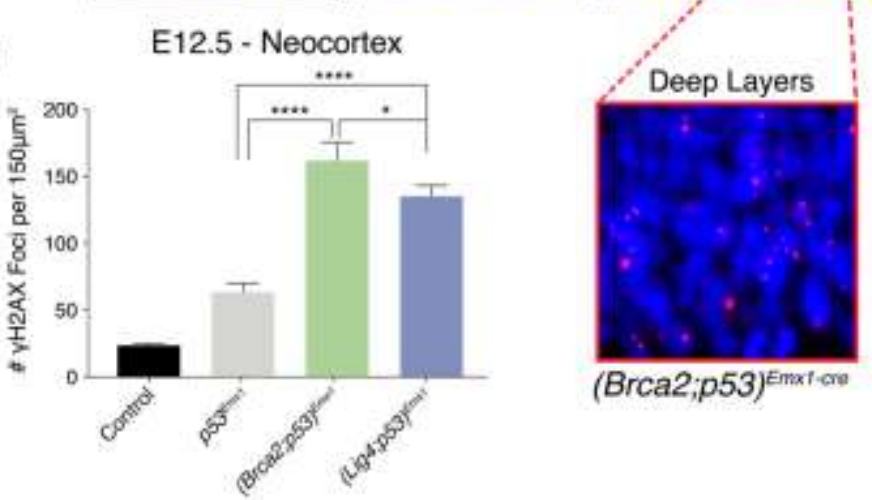

(Brca2;p53) Emxt-are
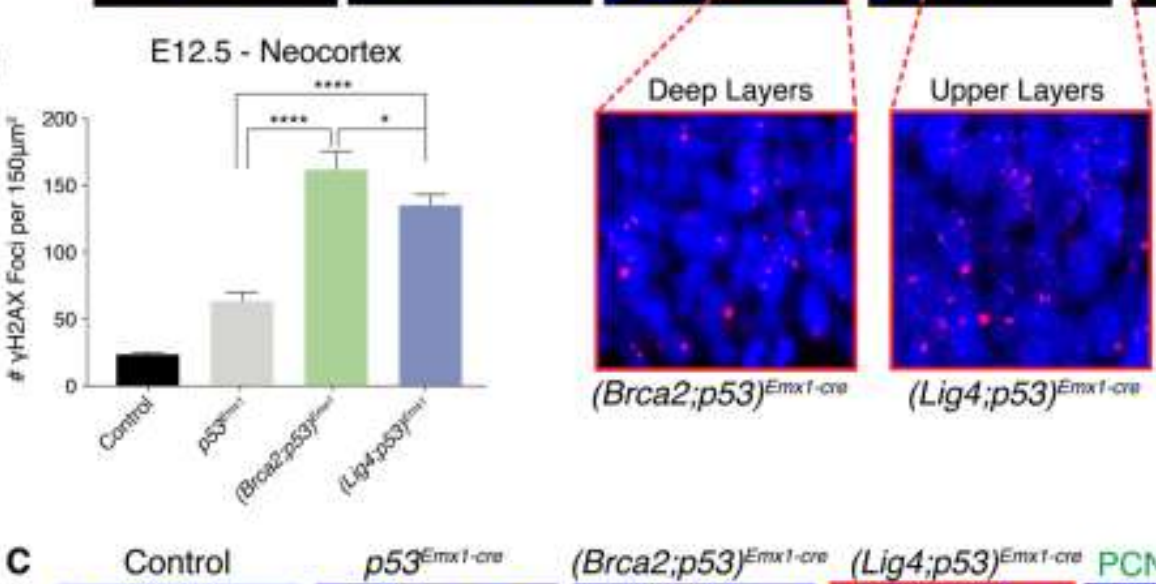

p53
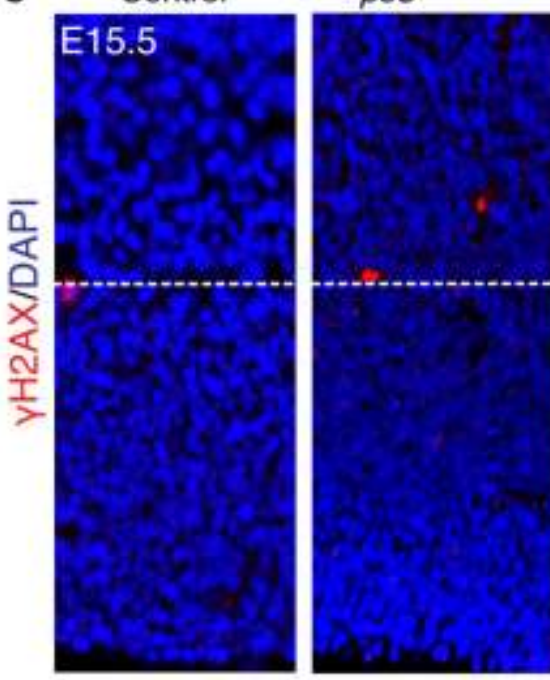

(Brca2;p53) Emx1-cre

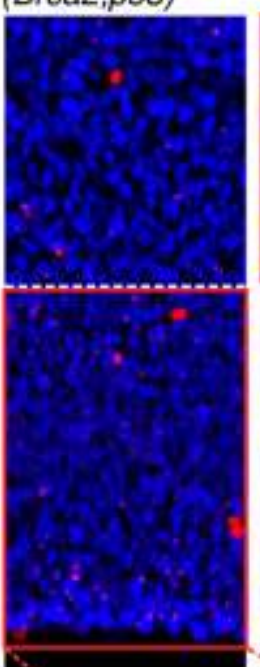

(Lig4;p53) $)^{\text {Emxical PCNA }}$

D

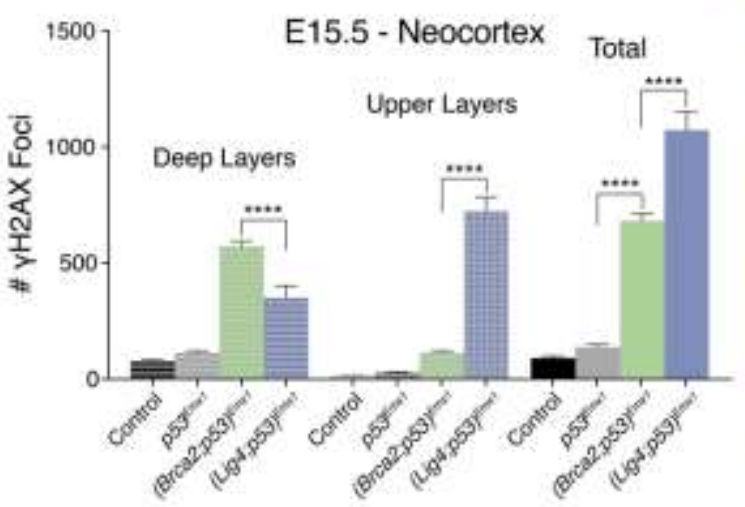

Deep Layers
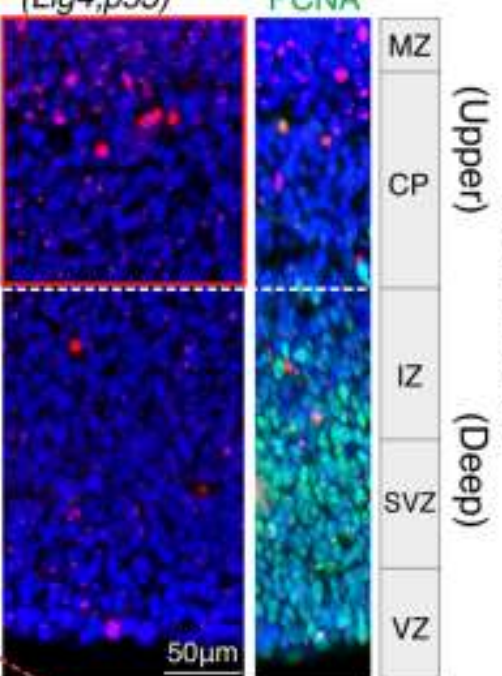

등

CP

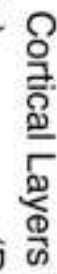
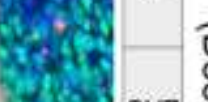

$x$ sas svi

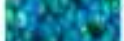

SVZ 믄
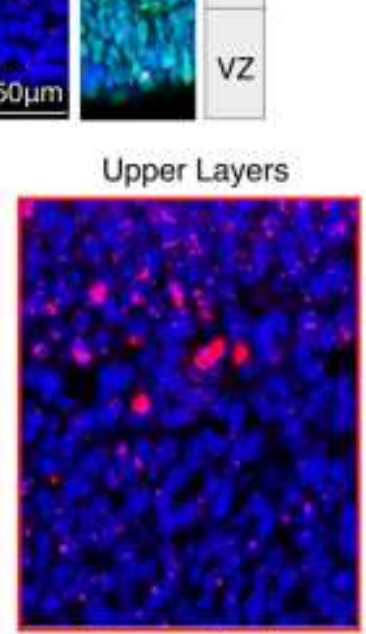

(Brca2:p53) Emx1-cre

(Lig4;p53) Emxt-cre 
born replicating progenitors (E12.5 and lower E15.5 cells) as they rely on a less errorprone mechanism to reduce risk of producing improperly repaired DNA [3, 4]. Therefore, it is logical that the majority of damage from Brca2 inhibition (HR-deficiency) would be identified in the deep highly proliferative subgroup of cells in the VZ, SVZ and IZ [48]. In contrast, NHEJ acts as the major repair pathway in post-mitotic, differentiating and mature neurons (Upper cells in E12.5/E15.5) [6]. Accordingly, the vast amount of DNA damage in the Lig4-deficient mice was found in the upper differentiated cortical cell layers. Surprisingly, the difference in the total amount and location of DNA damage didn't directly affect tumor latency or overall survival between either of the two DSBRdeficient mouse models. These findings significantly highlight the importance of proper DSBR in preventing DNA damage accumulation and gliomagenesis in the developing cortex.

\section{DNA Damage from DSBR-Deficiency with $p 53$ Inhibition Induces G2/M Checkpoint}

To confirm the effect DNA damage has on cortical progenitors, we assessed cell cycle checkpoint activation. Neural cells in the proliferative region of the ventricular zone (VZ) undergo interkinetic nuclear migration between E13 and E18, where the cells nuclear location is reflective of its cell cycle phase $[18,23]$. Cells in the S-phase reside at the basal surface, while cells in the G2/M-phase are located at the apical surface of the VZ, as illustrated in (Figure 3-7A). Apical cortical progenitors in M-phase can be identified using the histone $\mathrm{H} 3$ phospho-ser10 (pH3) marker. Additionally, we analyzed Tuj1, a marker for differentiated neurons, but noticed no observable difference between DSBRdeficient mice or controls at E12.5 or E15.5, however it did serve as a useful cortical layer marker (Figure 3-7B, D). We found a reduction of $\mathrm{pH} 3$-immumopositive M-phase cells only in the (Lig4;p53) ${ }^{\text {Emxl-cre }}$ neocortices at E12.5. However, a significant reduction in both the (Brca2;p53) $)^{\text {EmxI-cre }}$ and (Lig4;p53) EmxI-cre mutants was observed at E15.5 (Figure 3-7B-E). The lack of apical cortical cells in M-phase indicates an arrest in the cell cycle at this point, correlative with the high levels of the DNA damage present. Tumor RNA-seq analysis also identified enrichment of miotic, G2/M checkpoint phase, genes in both Brca2- and Lig4-deficient gliomas (Figure 3-7F and Figure 3-4D, respectively). Cell cycle arrest is designed to allow time for DDR and the cell will resist apoptosis unless it is unable to sufficiently repair the damage. However, the inhibition of p53 in this model does not allow induction of apoptosis, which accounts for the lack of cell loss throughout the cortex (data not shown) that we previously identified in mice with functional p53 (Figure 3-2A, D). The role p53 plays on the cell cycle is multifaceted as it also serves a critical function in controlling the G1/S-phase checkpoint [6], however since we inhibited $p 53$ we predicted this checkpoint would subsequently be inactivated. 5-bromodeoxyuridine (BrdU) incorporation experiments were performed on E15.5 mice to further examine the G1/S checkpoint in the neocortex. Basal progenitor cells in the G1/S phase can be identified as positive for BrdU. Staining for BrdU displayed no significant difference between $(B r c a 2 ; p 53)^{\text {Emxl-cre }}$ and control embryos (Figure 3-7G). Thus, supporting inactivation of $p 53$ inhibits the ability for the G1/Sphase checkpoint to be activated [204]. Together, these data indicate that loss of DSBR and $p 53$ inhibition induce high levels of DNA damage from replicative stress in cortical 
Figure 3-7. DNA damage from DSBR-deficiency induces G2/M cell cycle checkpoint.

(A) Illustration of the interkinetic nuclear migration process of progenitor cells within the SVZ and VZ as they progress through cell cycle. (B) Apical mitotic cortical progenitors in M-phase at E12.5 were identified using histone $\mathrm{pH} 3$ (Ser10) (white arrows - green cells). Tuj1 marks for differentiated neurons. (C) Quantification of $\mathrm{pH} 3$ positive cells at E12.5. $\mathrm{N} \geq 3$ animals per groups, $\geq 9$ images per group. $* * * * p<0.0001$; n.s., not significant. (D) Decreased mitotic positive apical progenitors at E15.5 in DSBR mutants using pH3. Tuj1 indicating cell layers of differentiated neurons. (E) Quantification of $\mathrm{pH} 3$ positive cells at $\mathrm{E} 15.5$. $\mathrm{N} \geq 3$ animals per groups, $\geq 9$ images per group. $* * p=0.0017$; n.s., not significant. (F) Upregulated genes and associated pathways involved in the G2/M-phase checkpoint in (Brca2;p53) Emx1-cre tumors compared to p53 $3^{\text {Emxl-cre }}$ controls (Enrichr). (G) BrdU incorporation was used to identify basal cortical progenitors in G1/S-phase at E15.5. Quantification of total BrdU positive cells in cortical layers; VZ, SVZ and IZ was also collected. $\mathrm{N}=1$ animal per group, $\geq 3$ images per group. Graphs represent mean values of replicates. n.s. = not significant Error bars indicate SEM.

Modified with open access permission. Azzarelli, R., F. Guillemot, and E. Pacary, Function and regulation of Rnd proteins in cortical projection neuron migration. Front Neurosci, 2015. 9: p. 19. 
D
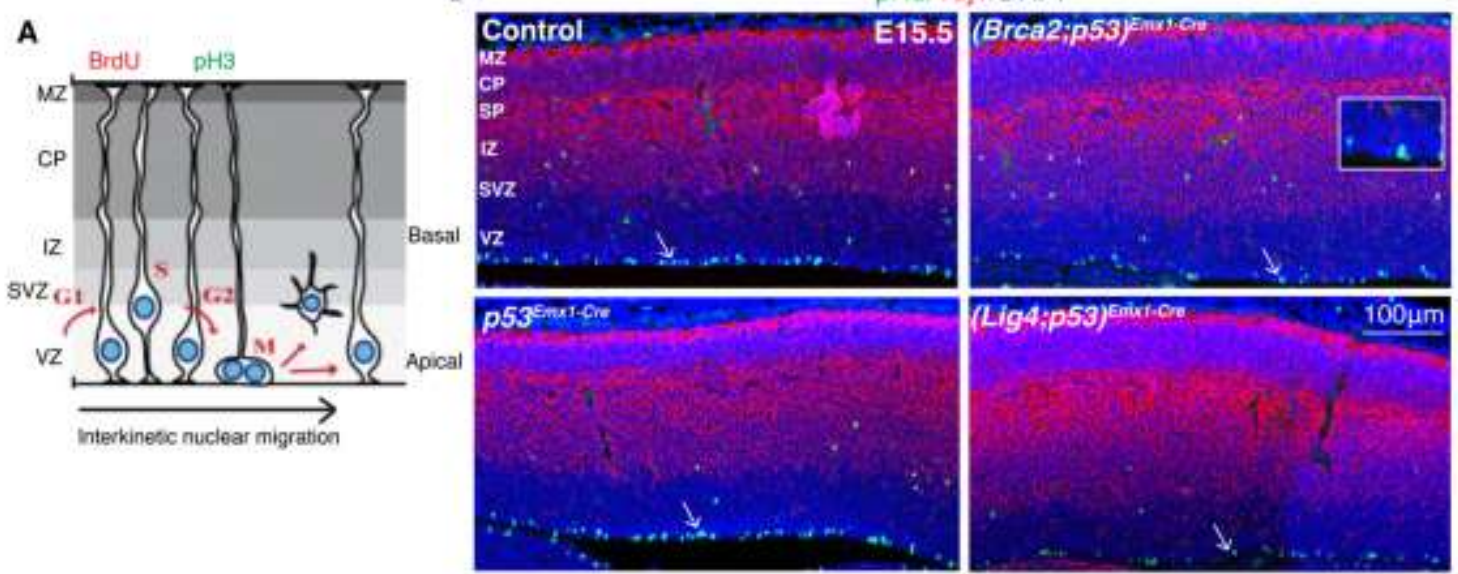

B
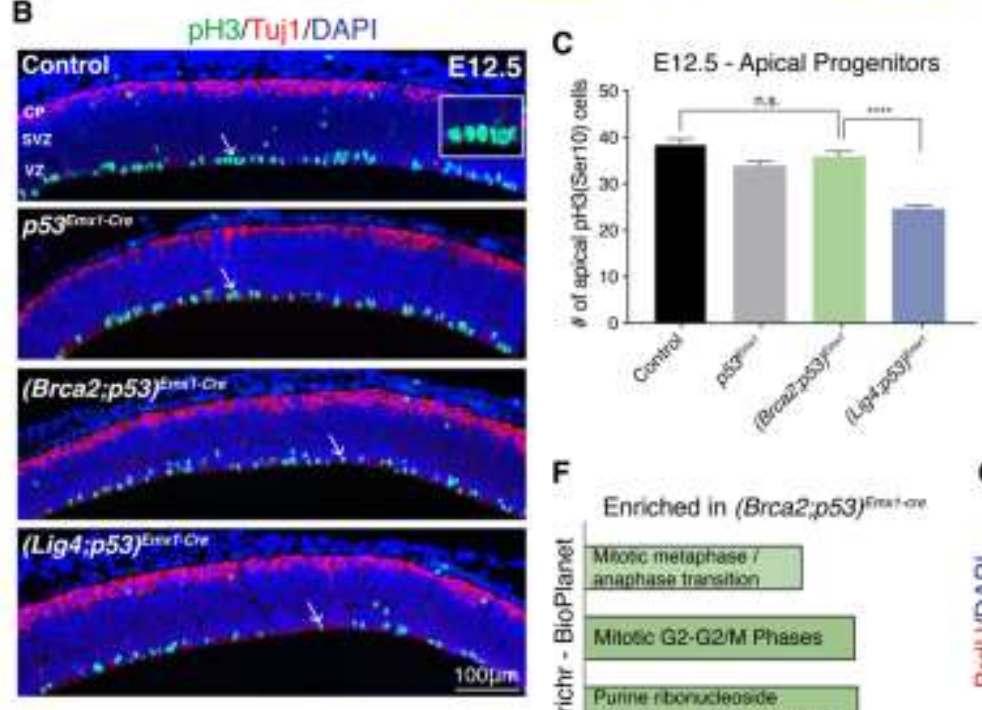

E

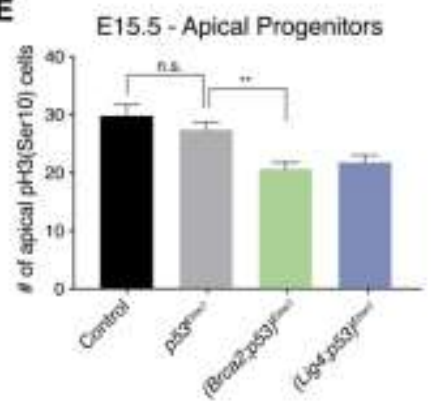

F
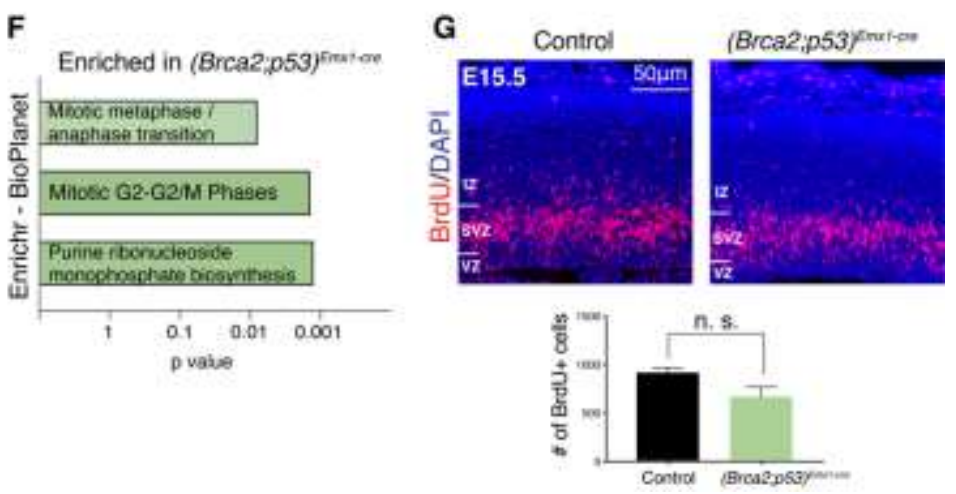
progenitors, which in turn activates the G2/M cell cycle checkpoint. Checkpoint activation allows sufficient time for errors in DNA repair to occur because of the coinciding DSBR-deficiency. Thus, these errors produce possible mutations which accumulate in the developmentally critical progenitor cell population resulting in increased tumorigenesis. 


\section{CHAPTER 4. H3K27M MODULATES BIVALENT GENE EXPRESSION WITH DSBR INACTIVATION ACCELERATING GLIOMAGENESIS}

Histone $\mathrm{H} 3$ mutations are critical events in pediatric high-grade gliomagenesis occurring in about $50 \%$ of cases [80-82]. The most common histone mutation found in these gliomas is $\mathrm{H} 3 \mathrm{~K} 27 \mathrm{M}$, which is identified in about $60 \%$ of DIPG and non-brainstem midline pHGGs [80]. A variety of other mutations are identified with H3K27M gliomas, however p53 mutations are by far the most frequent $(\sim 50 \%)$ [80-82]. Since the discovery of these oncohistones in pHGGs in 2012, the interest in developing representative models has taken off [81, 82, 154-157]. Recently, Suzanne Baker's lab created the first (H3.3) H3K27M ${ }^{\text {Lox-stop-Lox }}$ conditional knock-in mouse $[159,160]$. Their group showed that neonatal induction of H3K27M with activated PDGFR $\alpha$ and p53 loss accelerated diffuse brainstem gliomas that recapitulated DIPGs in a mouse model [160]. Using this new H3K27M mouse, we examined the consequence of this oncohistone in our glioma model in the context of cortical development, genome instability and cortex-specific gliomagenesis. In this chapter we discuss the results of expression of the H3K27M mutation in the context of $p 53^{\text {Emxl-cre }},\left(\right.$ Brca2;p53) ${ }^{\text {Emxl-cre }}$ and (Lig4;p53) ${ }^{\text {Emxl-cre }}$.

\section{H3K27M Mutation Produces Global Loss of H3K27me3 and Increases H3K27 Acetylation in Embryos}

We assessed what effect $\mathrm{H} 3 \mathrm{~K} 27 \mathrm{M}$ would have on the DSBR-deficient mice by breeding with $\mathrm{H} 3 \mathrm{~K} 27 \mathrm{M}^{\text {Lox-stop-lox }}$ conditional knock-in mice, resulting in the following cohorts; $(p 53 ; H 3 K 27 M)^{\text {Emxl-cre }},(\text { Brca2;p53;H3K27M) })^{\text {Emxl-cre }}$ and

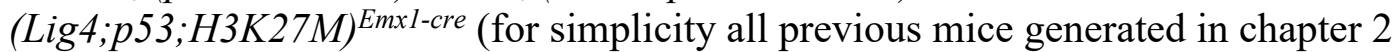
without the H3K27M mutation will be considered H3WT). Post-translational modifications (PTMs) of the histone mutation H3K27M in pHGG and as seen in other models classically reduce $\mathrm{H} 3 \mathrm{~K} 27$ tri-methylation (H3K27me3) and in some cases promote increased H3K27 acetylation (H3K27ac) [147, 160, 163, 205]. Examination of H3K27me3 expression levels in cortical embryonic tissue at both E12.5 and E15.5 showed a global loss of H3K27me3 in the neocortex of any H3K27M mutant compared to H3WT embryos, like (Brca2;p53) Emxl-cre. This data confirms H3K27M expression reprograms the H3K27me3 landscape in the cortex of this model (Figure 4-1A, B). To our knowledge this is the earliest reported endogenous gene activation of $\mathrm{H} 3 \mathrm{~K} 27 \mathrm{M}$ in a murine model $[154,156,157,160]$. Methylation at H3K27M is generally associated with gene silencing and acetylation is indicative of gene activation [147, 206]. We also found a modest increase of H3K27ac in upper and deep cortical layers of the H3K27M mutant mice (Figure 4-1C). Thus, activation of the H3K27M mutation via Emxl-cre recapitulates the consequence of this histone mutation seen in most other scenarios, independent of DSBR-deficiency. 
A

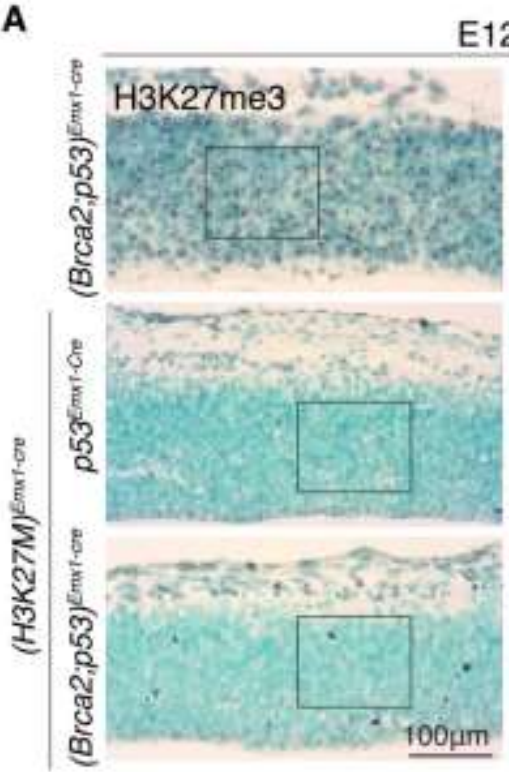

E12.5
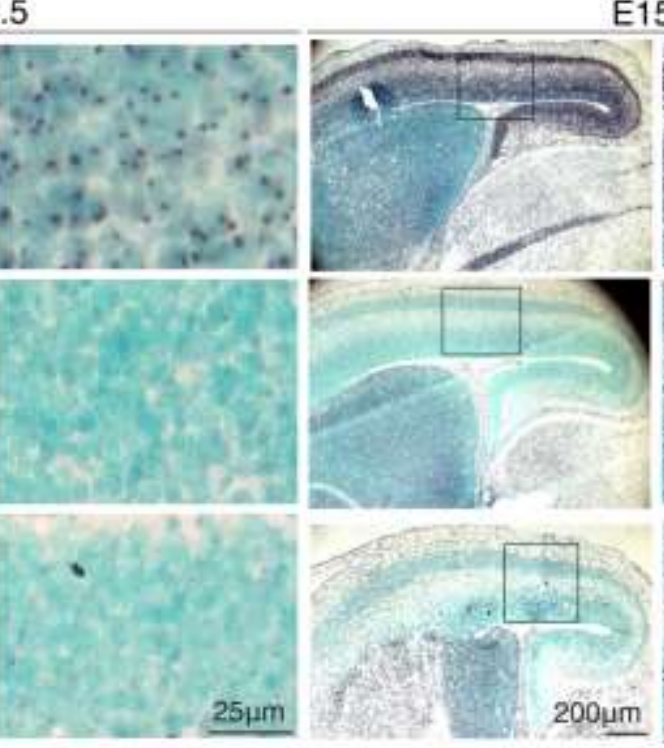

E15.5
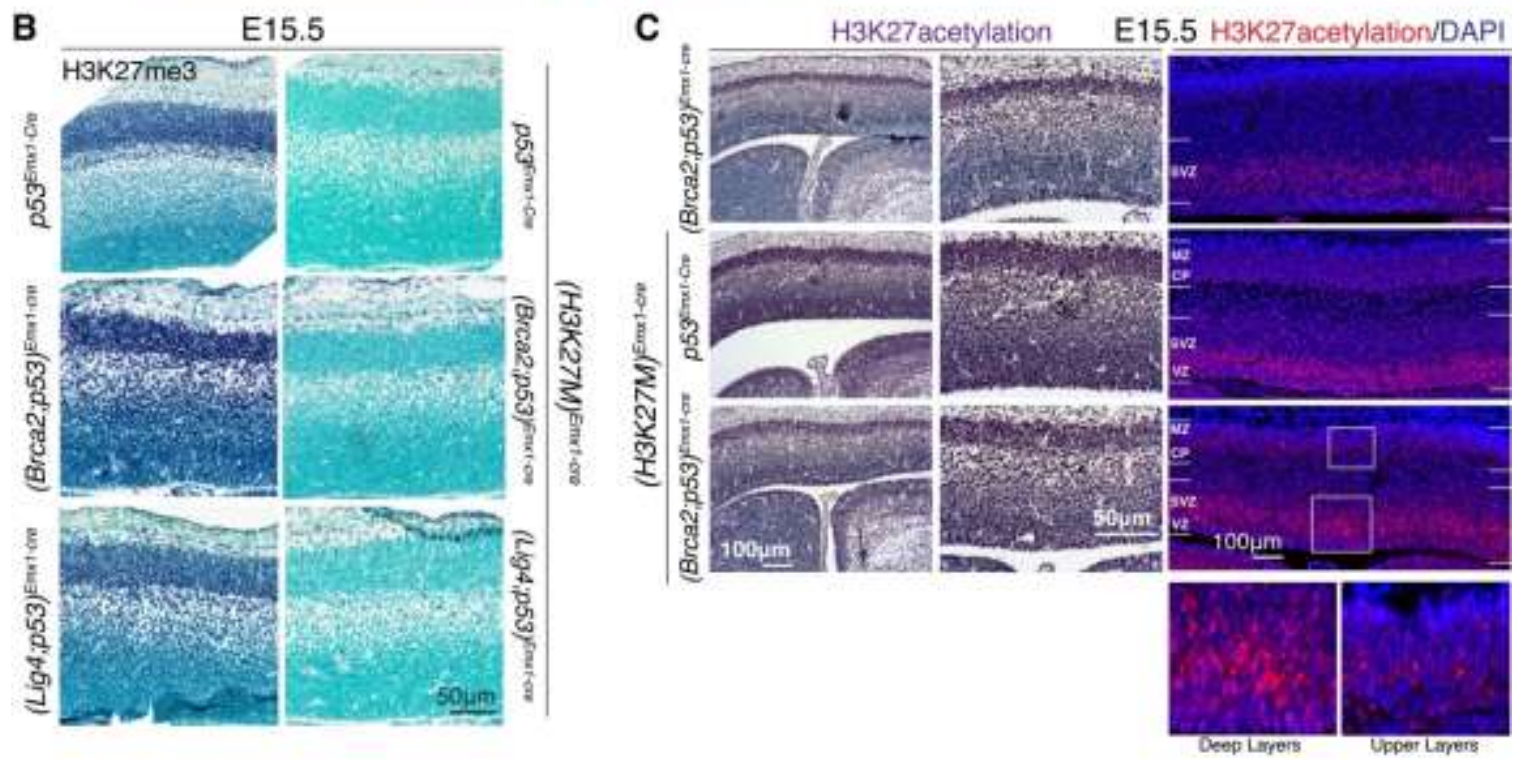

Figure 4-1. H3K27M mutation produces global loss of $\mathrm{H} 3 \mathrm{~K} 27 \mathrm{me} 3$ and increased H3K27acetylation in embryos.

(A) H3K27me3 is significantly reduced in all H3K27M mutant neocortices at E12.5 (left panels) and E15.5 (right panels) compared to (Brca2;p53) Emxl-cre mice. (B) All mouse cohorts containing H3K27M displayed almost complete abolition of H3K27me3 at E15.5 throughout the cortex. (C) H3K27acetylation is increased in H3K27M mutants compared to (Brca2;p53) Emx1-cre neocortices at E15.5 in upper and deep cortical layers (between white hash marks and closeups). Both colorimetric (left panels) and immunofluorescent (right panels) staining were performed for $\mathrm{H} 3 \mathrm{~K} 27 \mathrm{ac}$ expression. 


\section{H3K27M Accelerates Glioma Formation and Increases Tumor Diffusion}

To determine the impact of the H3K27M mutation on tumorigenesis we aged and monitored (p53;H3K27M) Emxl-cre,$(B r c a 2 ; p 53 ; H 3 K 27 M)^{\text {Emxl-cre }}$ and

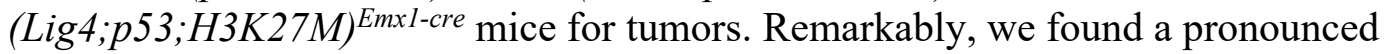
acceleration of tumor occurrence correlating with a decrease in survival (Figure 4-2A).

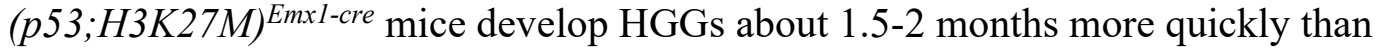
(Brca2;p53) ${ }^{\text {Emxl-cre }}$ or (Lig4;p53) ${ }^{\text {Emxl-cre }}$ mice and at an incidence rate of $\sim 69 \%$. The addition of DSBR-deficiency further decreased survival with (Lig4; $53 ;$ H3K27M) ${ }^{\text {Emx 1-cre }}$ mice dying on average about two months earlier than $(p 53 ; H 3 K 27 M)^{\text {Emxl-cre mutants and }}$ (Brca2;p53;H3K27M) ${ }^{\text {Emxl-cre }}$ mice having the lowest tumor-free survival at $\sim 6$ months. (Brca2;p53;H3K27M) ${ }^{\text {Emxl-cre }}$ mutants not only succumbed to gliomas quicker than

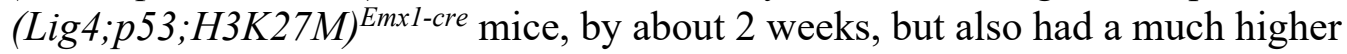
incidence of $\sim 90 \%$ compared to $\sim 67 \%$, respectively. On average the addition of H3K $27 \mathrm{M}$ with DSBR-deficiency accelerated tumorigenesis by about 4 months compared to their H3WT counterparts. Phenotypically, mice carrying the H3K27M mutation were smaller in overall size by about $30 \%$ less body weight at weaning age (21 days postnatal) compared to littermate controls, indicating a defect in early growth development (Figure 4-2B). Noticeably, the size discrepancy declined as mice aged into adults. In addition to initial delay in growth, mice with the H3K27M mutation also demonstrated extreme hyperactivity throughout their lifespan. This hyperactive behavior progressively slowed down within the last few weeks of life, with mice noticeably losing their fight-or-flight reactions and becoming slouched/hunched, dehydrated, almost immobile and moribund. These signs of mortality and additionally cranial swelling or a "domed head," are frequent among all of the glioma cohorts. The unique characteristic effects of H3K27M mice highlight some of the underlying problems associated with this mutation and the potential mechanisms that make it such a potent oncogene.

To determine the impact of expressing the H3K27M mutation in DSBR mutant background we assigned tumor diffusion scores by analyzing H\&E and PCNA immunohistochemistry (Figure 4-2C, D). Comparatively, all H3K27M tumors exhibited considerably higher diffusion scores (4 or 5) by more than $40 \%$ matched to H3WT gliomas. Both (Brca2; $53 ; H 3 K 27 M)^{\text {Emxl-cre }}$ and (Lig4;p53;H3K27M) ${ }^{\text {Emxl-cre }}$ gliomas were highly diffuse and infiltrative with increased cellularity and proliferative cell populations throughout almost the entire adult mouse cortices. Individually, (Lig4;p53;H3K27M)EmxI${ }^{c r e}$ mice displayed the greatest tumor cell diffusion, with $56 \%$ of analyzed tumors having a diffusion score of 5 compared to (Brca2;p53;H3K27M) ${ }^{\text {Emxl-cre }}$ gliomas that only had $17 \%$ with a score of 5 but $44 \%$ with a score of 4 (Figure 4-2C). Although

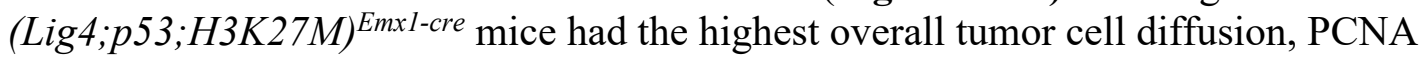
quantification identified significantly more proliferative positive cells in

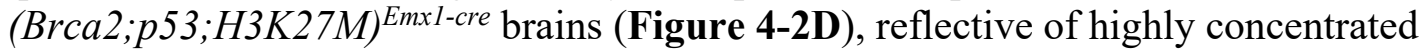
areas of PCNA in these mice (Figure 4-2C). When determining diffusion score, we based the analysis on the total area PCNA positive cells spread throughout the brain. Locationally, gliomas with H3K27M were similar to H3WT gliomas in that both primarily occur in the cortex and olfactory bulb with some increased diffusion into olfactory cortex, thalamus, hypothalamus, hippocampus and midbrain regions. This data 
Figure 4-2. H3K27M accelerates glioma formation and increases tumor diffusion. (A) Kaplan-Meier survival analysis displays decreased survival of mice with H3K27M. p53 $3^{\text {Emxl-cre }}(\mathrm{n}=15 / 44),(\text { Brca2;p53 })^{\text {Emxl-cre }}(\mathrm{n}=43 / 97),(\text { Lig4;p53 })^{\text {Emxl-cre }}(\mathrm{n}=45 / 108)$, $(p 53 ; H 3 K 27 M)^{\text {Emxl-cre }}(\mathrm{n}=23 / 33),($ Brca2;p53;H3K27M) EmxI-cre $(\mathrm{n}=65 / 72)$ and (Lig4;p53;H3K27M) ${ }^{\text {Emxl-cre }}(\mathrm{n}=68 / 102)$; Tumor incidence: $(\mathrm{n}=\#$ mice died of glioma / \# total mice), ${ }^{* * * *} \mathrm{p}<0.0001, \mathrm{n} . \mathrm{s} .=$ not significant $(\mathrm{p}=0.2326)$. (B) Graph displaying the difference in mouse weights at P21 in littermate controls showing decrease in mutants containing H3K27M, nondependent on gender. $* * * * p<0.0001, * p=0.0280$. (C) Glioma diffusion scores increased considerably in H3K27M brain tumors. H\&E and PCNA staining were used to determine diffusion score. White dashed lines outline primary tumor(s). PCNA indicates tumor cell proliferation and diffusion; numbers in bottom right denote diffusion score of representative images of HGGs. Least diffuse (1) to highest (5). Percentage of tumors analyzed with determined diffusion score. are shown. Scale bar, $\sim 1 \mathrm{~mm} .5^{\text {Emxl-cre }}(\mathrm{n}=4),(\text { Brca2;p53) })^{\text {Emxl-cre }}(\mathrm{n}=16),\left(\right.$ Lig4;p53) ${ }^{\text {Emxl-cre }}(\mathrm{n}=9)$, $(p 53 ; H 3 K 27 M)^{\text {Emxl-cre }}(\mathrm{n}=3),($ Brca2;p53;H3K27M)Emxl-cre $(\mathrm{n}=18)$ and $(L i g 4 ; p 53 ; H 3 K 27 M)^{\text {Emxl-cre }}(\mathrm{n}=9)$. (D) PCNA quantification of all glioma mouse samples. $* * * * p<0.0001, * * * p=0.0025$ (E) Reduction of H3K27me3 carries through development into tumors with H3K27M, independent on DSBR-deficiency. Scale bar, $1 \mathrm{~mm}$ for whole brain sections. 

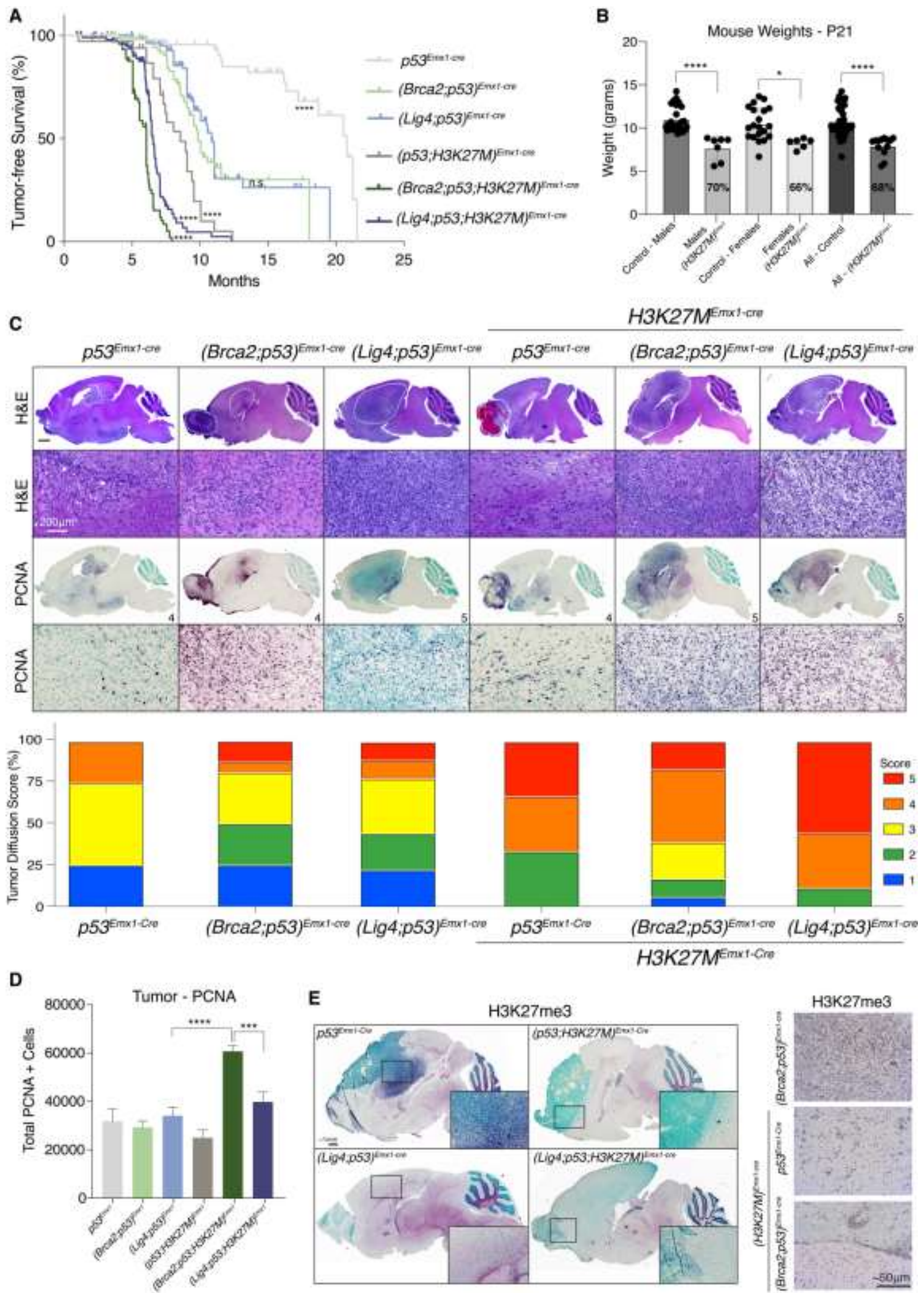
suggests the combinatorial effect of the H3K27M mutation increased tumor diffusion and probable overall glioma grade, supporting its known role in driving HGG formation in patients and other models $[81,82,155,160]$. All H3K27M mutant tumors observed contained similar amounts of H3K27me3 loss (Figure 4-2E), indicating the H3K27M mutation and its effect on PTMs is present throughout development into tumorigenesis in our model. Taken together, we observed the addition of H3K27M increases tumor incidence and diffusion and decreases survival, especially when DNA repair is compromised by concomitant inhibition of HR or NHEJ.

\section{H3K27M Enhances DNA damage Accumulation in the Mouse Neocortex}

We hypothesized that the histone mutation may affect chromatin conformation leading to increased DNA damage susceptibility as part of the mechanism leading to gliomagenesis. To assess the levels of DNA damage introduced by the expression of the $\mathrm{H} 3 \mathrm{~K} 27 \mathrm{M}$ mutation in DSBR-deficient mutants, we examined $\gamma \mathrm{H} 2 \mathrm{AX}$ staining to quantify levels of DSBs during corticogenesis. At E12.5, the expression of the H3K27M mutation in the $(B r c a 2 ; p 53 ; H 3 K 27 M)^{E m x l-c r e}$ mouse resulted in a significant increase in $\gamma \mathrm{H} 2 \mathrm{AX}$ foci compared to all other cohorts, suggesting H3K27M is further inhibiting DNA repair in the early stages of cortical development where cells are highly replicative (Figure

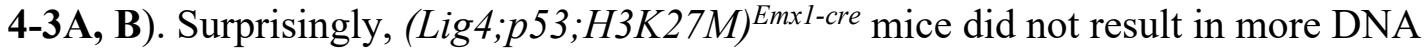
damage at E12.5 than (Lig4;p53) Emxl-cre embryos, indicating a preferential importance of $\mathrm{H} 3 \mathrm{~K} 27 \mathrm{M}$ and HR in the earlier stages of embryogenesis. We did observe a subtle cortical cell layer difference after DNA damage that was previously identified at E12.5 between Brca2-deficient (lower) and Lig4-deficient (upper) mice (Figure 4-3A and 3-6A). It appears that (Lig4;p53;H3K27M) Emxl-cre mice have increased DNA damage in the upper layers, while (Brca2;p53;H3K27M) ${ }^{E m x l-c r e} \gamma \mathrm{H} 2 \mathrm{AX}$-foci are distributed throughout the proliferative cells at this stage (Figure 4-3A). Most intriguingly, at the E12.5 developmental stage, there was a significant amount of $\gamma \mathrm{H} 2 \mathrm{AX}$-foci present in $\left(\right.$ p53;H3K27M) ${ }^{\text {Emxl-cre }}$ cortices (Figure 4-3A, B). These mice have intact DSBR, but sustained significantly more DNA damage at E12.5 than (Lig4;p53) Emxl-cre mice, suggesting that the histone mutation and its consequential effect on the chromatin conformation may be playing a role in preventing or delaying appropriate DNA repair [207-209]. Further, we observed slightly lower amounts of DNA damage in H3K27M EmxIcre (functional p53) neocortices at E12.5 compared to $(p 53 ; H 3 K 27 M)^{\text {Emxl-cre }}$ (data not shown), indicating that $\mathrm{p} 53$-mediated DNA damage response may be regulating repair.

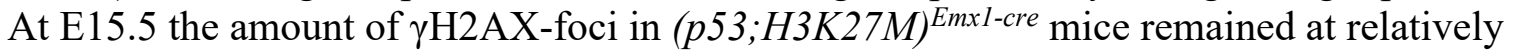
the same level, with only a slight increase in the deep cortical layers (Figure 4-3C, D). This highlights the importance of H3K27M in the highly proliferative cortical cell population present in the lower layers. The addition of H3K27M with the DSBRdeficient mutants further exacerbated the cortical spatial difference of DNA damage that we previously identified between (Brca2;p53) ${ }^{\text {Emxl-cre }}$ and (Lig4;p53) ${ }^{\text {Emxl-cre }}$ mice at E15.5. (Brca2;p53;H3K27M) Emxl-cre embryos displayed significantly more $\gamma \mathrm{H} 2 \mathrm{AX}$-foci in deep cortical layers. While (Lig4;p53:H3K27M) ${ }^{\text {Emxl-cre not only had significantly more }}$ DSBs in the upper layers but resulted in the greatest amount of DNA damage accumulation total throughout the entire cortex at E15.5 (Figure 4-3D). Interestingly, 
Figure 4-3. H3K27M enhances selective DNA damage accumulation in the mouse neocortex.

(A) DNA damage $\left(\gamma \mathrm{H} 2 \mathrm{AX}\right.$-foci) increases in $(p 53 ; H 3 K 27 M)^{\text {Emxl-cre }}$ and

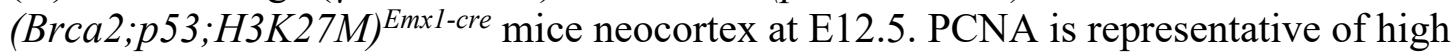
proliferation throughout cortex at this state. Side box and dashed white line indicates approximate division of upper and deep cortical layers. Lower panels have DAPI subtracted to highlight $\gamma \mathrm{H} 2 \mathrm{AX}$-foci. (B) Total number of $\gamma \mathrm{H} 2 \mathrm{AX}$-foci quantified in $150 \mu \mathrm{m}^{2}$ area. $\mathrm{N} \geq 3$ animals per groups, $\geq 9$ images per group. ${ }^{*} \mathrm{p}<0.0306,{ }^{*} \mathrm{p}<0.0150$, $* * * * \mathrm{p}<0.0001$, n.s. $=$ not significant. (C) DNA damage is cumulative at E15.5 in H3K27M mutants with selective differences in cortical layers. (Lig4;p53;H3K27M) ${ }^{\text {Emx I- }}$ cre mice have significantly higher levels of damage in upper layers, while

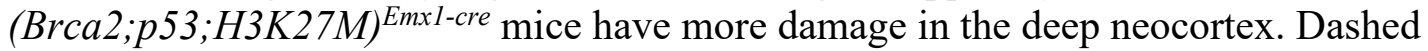
white line indicates division of upper and deep cortical layers. Lower panels have DAPI subtracted to highlight $\gamma \mathrm{H} 2 \mathrm{AX}$-foci. Pan-nuclear $\gamma \mathrm{H} 2 \mathrm{AX}$ stain may be indicative of cell death or non-specific background. (D) Number of $\gamma \mathrm{H} 2 \mathrm{AX}$ foci quantified for upper and deep layers and total neocortex combined. Deep layers: $330 \mu \mathrm{m}^{2}$ area quantified; Upper layers: $200 \mu \mathrm{m}^{2}$ area quantified; Total all layers: $550 \mu \mathrm{m}^{2}$ area quantified. $\mathrm{N} \geq 3$ animals per groups, $\geq 9$ images per group. $* * * * p<0.0001, * * * p=0.0001, * * p=0.0065$, $* * p=0.0031, * p=0.0446$, n.s. $=$ not significant. All graphs represent mean values of replicates. Error bars indicate SEM. Abbreviations: VZ: ventricular zone, SVZ: subventricular zone, IZ: intermediate zone, CP: cortical plate, MZ: marginal zone. 

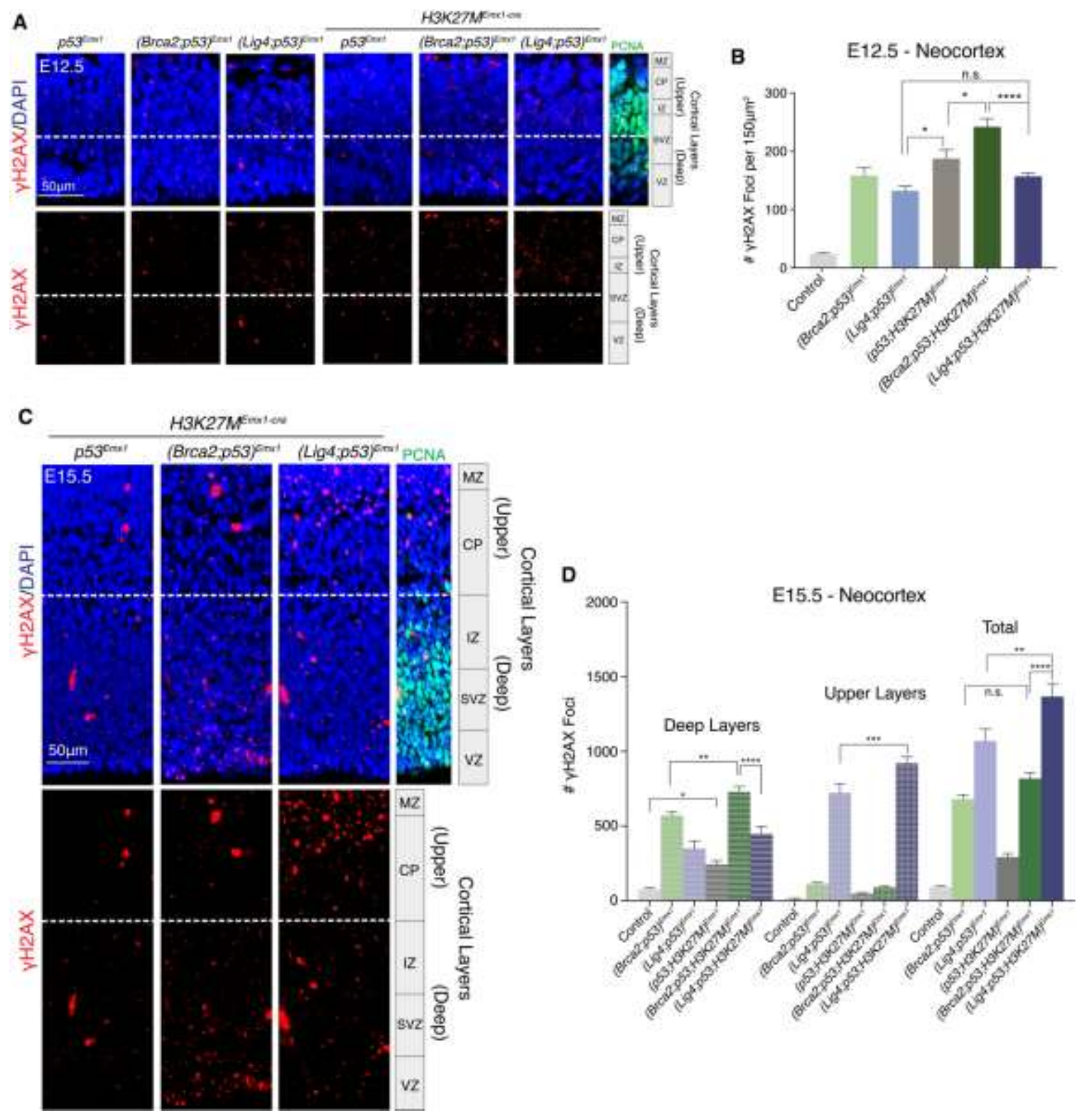
even with the significant increase of $\gamma \mathrm{H} 2 \mathrm{AX}$-foci in the deep cortical layers of (Brca2;p53;H3K27M) Emxl-cre mice, this did not result in enough damage to change the total amount compared to (Brca2;p53) ${ }^{\text {Emxl-cre }}$. These data together, suggest that H3K27M effect on genome stability aligns with the importance of the particular DSBR pathway at the developmental time period which it is the preferred repair mechanism. In short, H3K27M increases DNA damage with Brca2-deficency (HR) in the deep earlier born cortical progenitors, while having a similar effect with Lig4-deficency (NHEJ) in the upper, more differentiated, cell populations.

\section{DNA Damage from H3K27M Induces G2/M Checkpoint in Cortical Progenitors}

With the observed increase in DNA damage from mice carrying the $\mathrm{H} 3 \mathrm{~K} 27 \mathrm{M}$ mutation we further investigated the effect of this on G2/M checkpoint activation using a similar method as described previously utilizing the mitotic phospho-H3 marker in the neocortex. All H3K27M groups displayed significantly less mitotic positive apical cortical progenitors compared to control and (Brca2;p53) Emxl-cre mice at E12.5 (Figure 4-4A). Interestingly, (Brca2;p53;H3K27M) ${ }^{\text {Emxl-cre }}$ significantly activated the G2/M checkpoint, likely reflecting the increased DNA damage from the histone mutation. This same effect was not seen in (Lig4;p53:H3K27M) ${ }^{\text {Emxl-cre }}$ mice as these displayed almost identical amounts of $\mathrm{pH} 3$ positive cells as (Lig4;p53) EmxI-cre cortices, aligning with the similar location and amount of DNA damage seen in both of these mutants at E12.5 and E15.5 (Figure 4-4B). At E15.5 the greatest G2/M checkpoint activation in apical progenitors was observed in the (Brca2;p53;H3K27M) ${ }^{\text {Emxl-cre }}$ cortex (Figure 4-4B), suggesting again the lower earlier born cortical progenitors are the greatest effected by extra damage from H3K27M. Work done by Wieland Huttner's group and others have shown that directly reducing $\mathrm{H} 3 \mathrm{~K} 27 \mathrm{me} 3$ on the Eomes locus, a key basal progenitor regulator of Tbr2 in NSCs of mice, not only reduces the number of Tbr2 positive cells in the cortex but also directly decreases the amount of basal progenitors undergoing mitosis $[106,210,211]$. Interestingly, a small population of basal progenitors in the SVZ undergoing mitosis also stain positive for $\mathrm{pH} 3$. We found in the $\mathrm{H} 3 \mathrm{~K} 27 \mathrm{M}$ mice, where we show a global decrease in cortical H3K27me3 (Figure 4-1A, B), that this decrease in H3K27me3 directly correlates the loss of basal mitotic positive cells and Tbr2 positive cells (Figure 4-4B, C). The loss of Tbr2-positive cells was measured throughout the IZ, SVZ and VZ cortical layers in H3K27M mice compared to controls (Figure 4-4C). This suggests that epigenetic modifications, like repressive histone methylation, contribute to regulation of the developing neocortex. Lastly, BrdU incorporation was again measured to identify potential differences in basal progenitors undergoing the G1/S-phase transition at E15.5 in the H3K27M mutants (Figure 4-4D). Analyzing both the intermediate zone and all other BrdU positive cells individually found no significant reduction or effect from the histone mutation, which further supports that loss of p53 prevents activation of the G1/S checkpoint. Taken together, these data indicate H3K27M further exacerbates DNA damage in the neocortex which induces the G2/M checkpoint in the basal and apical progenitor cells. The delay and prolongation of the cell cycle at this critically important neural expansion time period suggest that epigenetic modifications from H3K27M contribute to genome instability, improper cortical development and ultimately 
Figure 4-4. DNA damage from H3K27M induces G2/M checkpoint in cortical progenitors.

(A) Apical cortical progenitors in M-phase at E12.5 were identified using histone $\mathrm{pH} 3$ (Ser10) (green) and neuronal differentiation marker Tuj1 (red) was used for cortical layer identification. ( $\mathrm{n} \geq 3$ animals per groups, $\geq 9$ images per group). $* * * * p<0.0001, \mathrm{n}$. $\mathrm{s}$. $=$ not significant. (B) G2/M checkpoint activation at E15.5 in basal (white arrows) and apical (red arrows) progenitors were analyzed and quantified individually. ( $\mathrm{n} \geq 3$ animals per groups, $\geq 9$ images per group). ${ }^{*} p<0.05, * * p<0.01$. (C) Tbr2 identifies intermediate basal progenitors progressing through the VZ, SVZ and IZ at E15.5. Tbr2 positive cells are reduced in $\mathrm{H} 3 \mathrm{~K} 27 \mathrm{M}$ mutants. ( $\mathrm{n}=1$ animal per group, $\geq 3$ images per group. $* p=0.0323, * * * p=0.0005$. (D) BrdU incorporation was used to identify basal cortical progenitors in S-phase proliferating at E15.5. BrdU positive cells were quantified in the IZ only or all lower layers together (VZ, SVZ, and IZ). ( $n=1$ animal per group, $\geq 3$ images per group. n. s. = not significant. All graphs represent mean values of replicates. Error bars indicate SEM. 
A

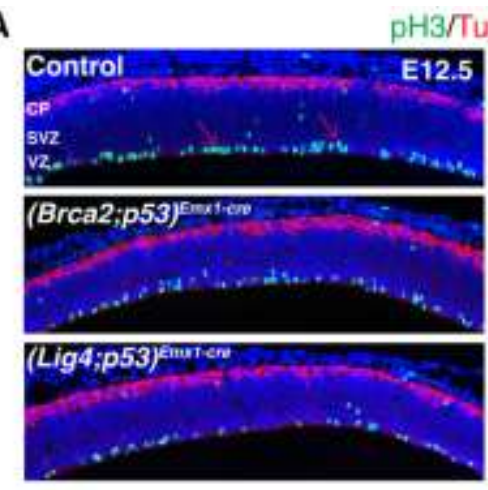

H3/Tuj1/DAPI

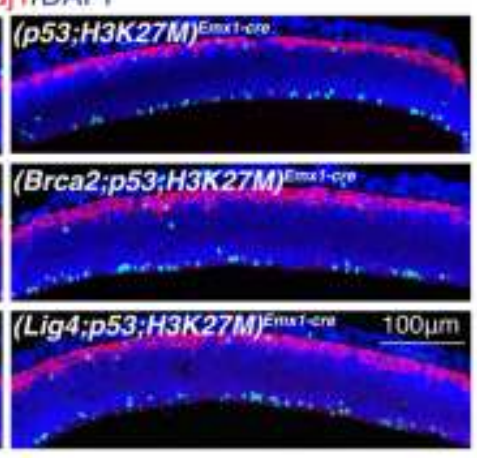

B
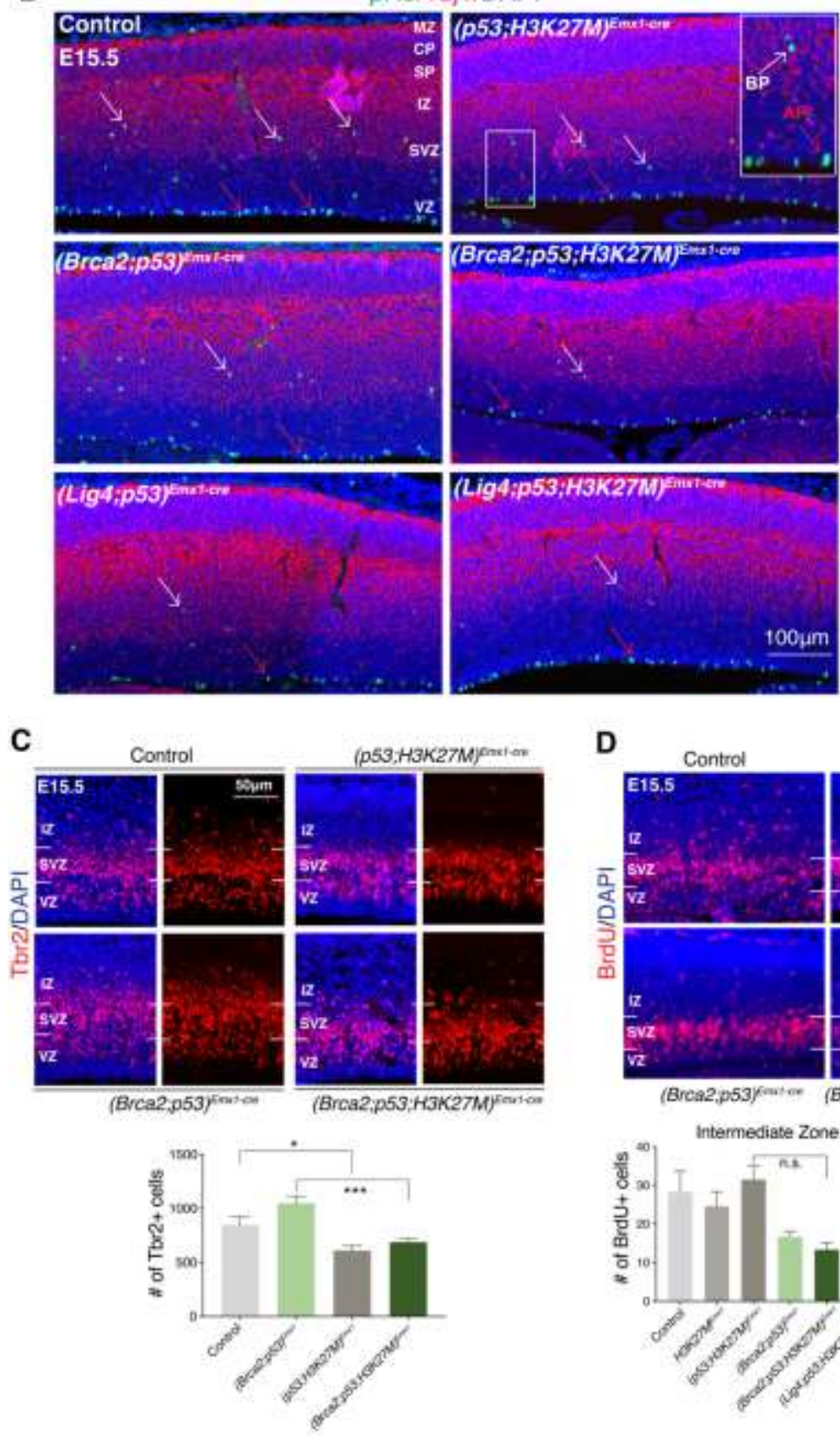

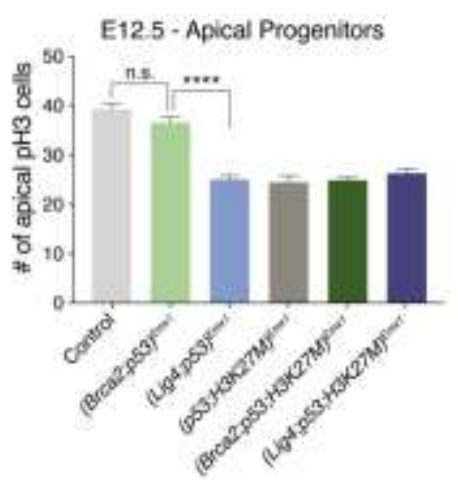

E15.5 - Apical Progenitors
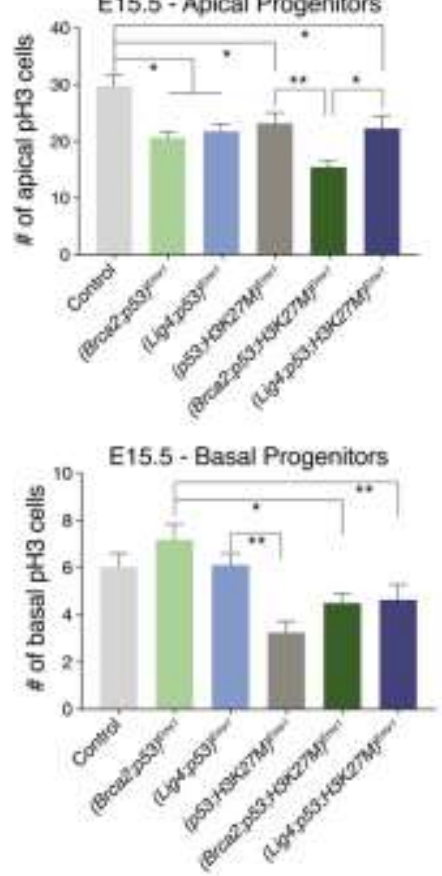

D
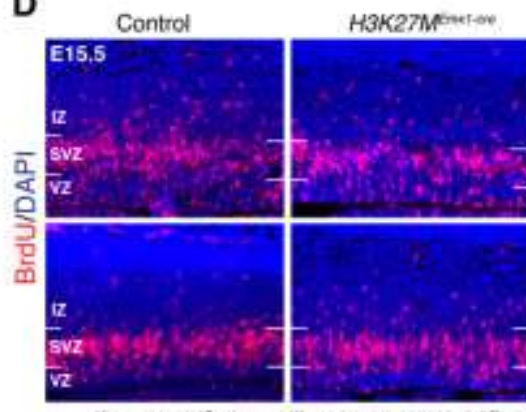

(p53,H3K27M) Eiveter

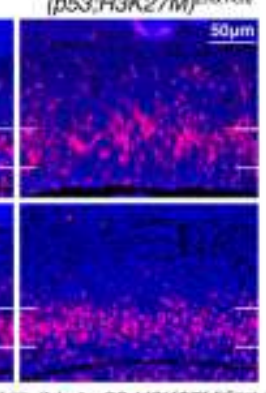

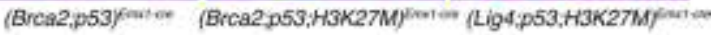
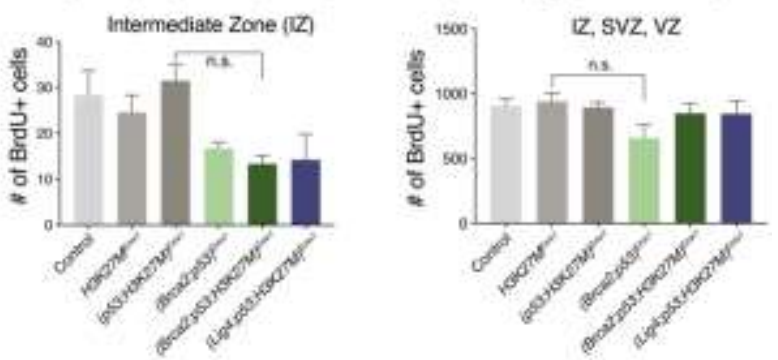
accelerated tumorigenesis.

\section{H3K27M Gliomas Exhibit Unique Gene Expression Changes That Recapitulate Signatures Identified in pHGGs}

We performed RNA-sequencing on (p53;H3K27M) $)^{\text {Emxl-cre, }}$ (Brca2;p53;H3K27M) $)^{\text {Emxl-cre }}$ and (Lig4;p53:H3K27M) ${ }^{\text {Emxl-cre }}$ gliomas to assess gene expression changes that may underpin the function of $\mathrm{H} 3 \mathrm{~K} 27 \mathrm{M}$ driven malignancy effects. Unsupervised hierarchical clustering of the most differentially expressed (up- or downregulated) genes revealed H3K27M tumors group together with distinct gene expression patterns compared to tumors without the histone mutation (H3WT) (Figure 4-5A, B). Mouse gliomas with H3WT displayed less consistent grouping, suggesting H3K27M may be reducing tumor heterogeneity (Figure 4-5A). Examination of genes with the greatest differential expression uncovered uniquely enriched gene sets based on the presence or absence of H3K27M (Figure 4-5B). Plagl (oncogene), Hopx (neurodevelopment), Eya4 (DNA repair) and Slit2 (axon guidance and neuronal migration) are among the most significantly upregulated in H3K27M tumors and show clear expression shift from H3WT gliomas, as shown in violin plots (Figure 4-5B, upper panel). Conversely, some of the most downregulated genes in H3K27M gliomas were Irx3/5 (embryonic neural development), Foxd2 (neurodevelopment), Gata6 (cell differentiation and bone morphogenetic protein (BMP) signaling), Hoxa 7 (embryonic development), En1/2 (embryonic development) and Six2 (proliferation and migration) (Figure 4-5B, lower panel). Many of these genes, including Plag1, Eya4, Slit1/2 and Gata6, are identified and emphasized as prominent factors in other similar H3K27M models $[155,157,160,162]$. One particular group of downregulated genes from H3K27M signatures are the Hoxa gene cluster (Hoxa2-7, and 10), a subset of homeobox genes directly involved in transcriptional regulation and differentiation (Figure 4-5B, C) [212]. Downregulation of homeobox genes indicates H3K27M is potentially repressing developmentally important genes to favor tumorigenesis.

When examining the relative change in gene expression between gliomas containing the H3K27M mutation and those without the mutation (H3WT), we found a greater overall average gene expression in the gliomas carrying H3K27M, with 658 genes showing increased expression and 367 displaying decreased expression (Figure 4-5D). This aligns with evidence supporting H3K27M mutations being linked to increased gene expression due to the global loss of the repressive H3K27me3 mark [213]. Amongst the most upregulated genes in H3K27M gliomas were oncogenes Egfr and Lin28b, tumor suppressor $C d k 6$, and Eyal/2, which are linked to DNA repair (Figure 4-5E). Consistent with the known role that the polycomb repressive complexes, PRC1 and PRC2, play in modulating H3K27me3 [150, 213, 214], specific components of these complexes were identified through the ChIP-sequencing Enrichment Analysis (ChEA) database [215]. Upregulated genes of these complexes in H3K27M gliomas were Ring $1 b$ and Bmil (PRC1) and Ezh2, Suz12, and Jarid2 (PRC2) (Enrichr - ChEA) [176] (Figure 4-5F).

Interestingly, PRC1 components have also been linked to stable repression of 
Figure 4-5. H3K27M gliomas exhibit unique gene expression changes that recapitulate signatures identified in pHGGs.

(A) Unsupervised clustering of genes identified by ANOVA of all HGG groups reveals H3K27M tumor samples increasingly cluster by genotypes in a heatmap. $p 53^{\text {Emx } 1 \text {-cre }}(\mathrm{n}=$ 6), (Brca2;p53) Emx1-cre $(\mathrm{n}=16),\left(\right.$ Lig4;p53) Emx1-cre $(\mathrm{n}=10),(\text { p53;H3K27M })^{\text {Emxl-cre }}(\mathrm{n}=3)$, (Brca2;p53;H3K27M) Emxl-cre $(\mathrm{n}=18)$ and $(\text { Lig4;p53;H3K27M) })^{\text {Emxl-cre }}(\mathrm{n}=9)$. False discovery rate cutoff $<0.05, \mathrm{p}<0.0001$ (B) Heatmaps and violin plots showing the average RNA-seq expression signal for the most up- and downregulated genes. The width of each violin displays how common expression levels are, with the widest portion corresponding to the mode average. Unpaired student t-test (upregulated; $3.05 \times 10^{-39}$ ), (downregulated; $1.69 \times 10^{-90}$ ). (C) Heatmap showing unsupervised clustering of all Hox genes expression comparing all H3K27M and H3WT gliomas. Hoxa genes, highlighted in red box, with higher expression in H3WT tumors. (D) MA-plot graphs the relative change in gene expression between gliomas containing the H3K27M mutation and those without it (H3WT). Genes meeting a LogFC change of 0.5 and a p-value of 0.05 are labelled in blue, while those with no significant change are black. There are 658 genes upregulated and 367 downregulated. (M: LogFC and A: Average Log Expression). (E) Volcano plot displays the change in gene expression compared to significance of expression change of all H3K27M tumor samples compared to all H3WT samples with representative gene names indicated. (F) Enrichr analysis indicates significantly associated enriched pathways of H3K27M upregulated genes. ChEA - ChIP; PRC1/2 upregulated targets and known binding partners of the genes in these two complexes. PANTHER; axon guidance affected via Slit/Robo pathway. Gene Perturbations; models relevant to $\mathrm{H} 327 \mathrm{M}$ mutations with other common upregulated genes. (G) GSEA using the most upregulated genes from all the H3K27M mouse gliomas applied to a gene set for a H3K27M human sample that was identified from the gene perturbations database (H3F3A K27M GSE34824). Heatmap shows the relative expression of the top 27 genes. 


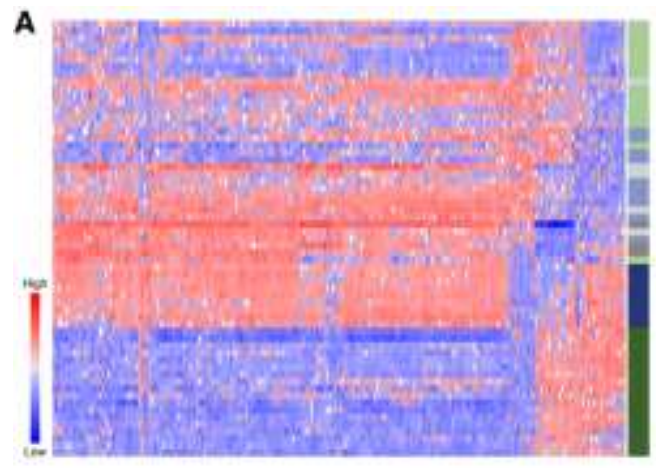

B

ansonition

$=($ Brca2,p53)

$=(L i g 4 ; 053)^{\text {surias }}$

$=(053$; H3K27M)

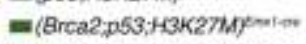

= (Lig4, p53,H3К27Mpetion

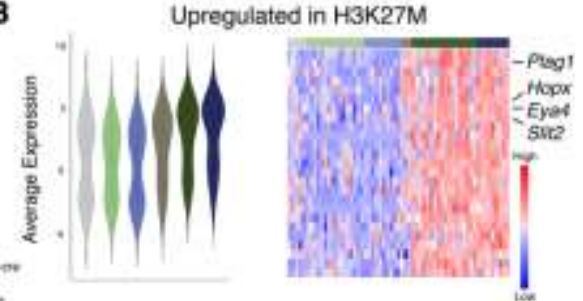

Downregulated in H3K27M

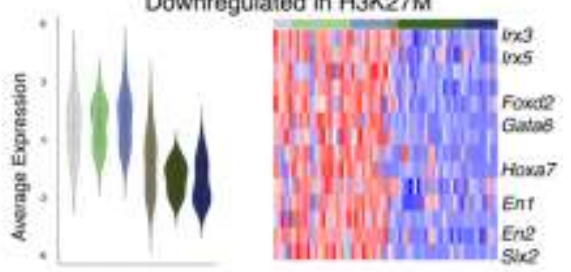

D All H3K27M vs All H3WT

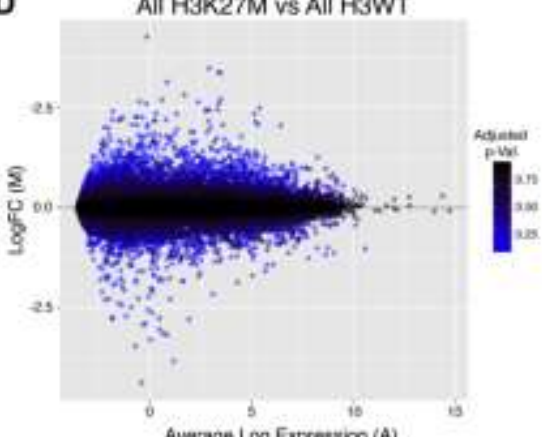

E All H3K27M

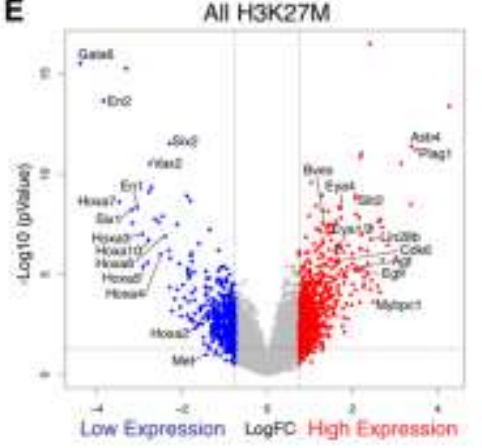

\section{F ChEA} Bmin

Ezh2

Jarda

Ping10

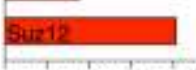

15-10 st:20 $15 \cdot 30+4+40$
p value

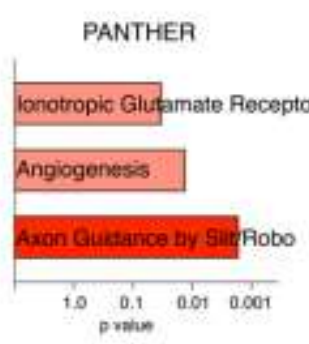

Gene Perturbations - GEO Up H3F3A - G34R - Human EZH2KD - Mouse PDGFAA HUMB HMGAZ KD - Hüman

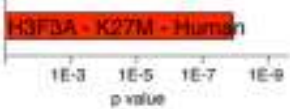

G s $\mathrm{H} 3 \mathrm{WT}$ - Н3К27M

Upregulated in All H3K27M H3F3A K27M - Mutation of Key Residues

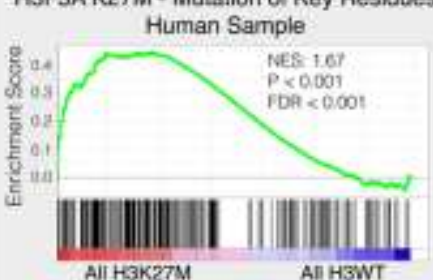

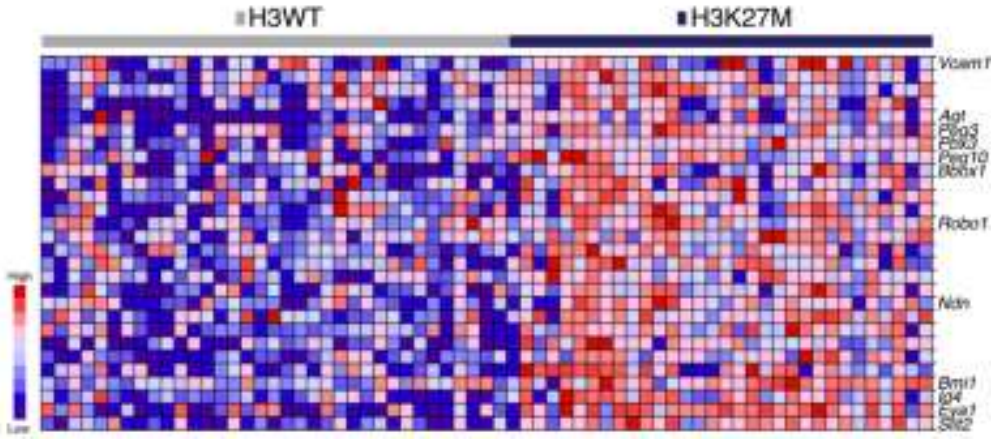


Hoxa genes [216]. We found key Slit-Robo pathway genes (Slit1/2 and Robo1/2) were upregulated in $\mathrm{H} 3 \mathrm{~K} 27 \mathrm{M}$ tumors which directly alter axon guidance, neuronal systems/development and netrin-1 signaling (identified through the PANTHER database, which classifies proteins and correlating genes) [217] (Figure 4-5F). Using another database that looks at RNA expression profiles from transcription factor perturbations in thousands of cell lines and tissues (GEO Up) [218], we uncovered a high overlap of highly expressed genes in other $\mathrm{H} 3 \mathrm{~K} 27 \mathrm{M}$ related samples compared to the most upregulated genes in the H3K27M gliomas (Figure 4-5F). These included human patient samples with mutations in $H 3 G 34 R$ (recurrent histone mutation), PDGFR (frequently overexpressed in pHGGs) and HMGA2 (epigenetic modifier) and mouse model samples, like an EZH2 KD. Furthermore, GSEA showed that the most upregulated genes from the H3K27M tumors were significantly enriched in a H3K27M human sample that we identified from the gene perturbations database (H3F3A K27M GSE34824), which was one of the original pHGG samples Nada Jabado's group discovered to have common histone mutations (Figure 4-5F, G) [82]. From this we identified a unique subset of shared upregulated genes, including Vcam 1 (vascular cell adhesion molecule-1), Agt (angiotensinogen) $P b x 3$ (pre-B-cell leukemia homeobox), $P$ eg3/10 (common abnormal expression in gliomas), Id4 (inhibitor of differentiation 4 - frequently overexpressed in gliomas), $N d n$ (necdin - associated with Prader-Willi syndrome), Bmil (proto-oncogene), Slit2 and Eyal/2/4. All of these genes have been shown to play important roles in gliomagenesis and/or neurodevelopment [219-229]. This data taken together, suggest gene expression changes associated with $\mathrm{H} 3 \mathrm{~K} 27 \mathrm{M}$ in a glioma mouse model recapitulate $\mathrm{H} 3 \mathrm{~K} 27 \mathrm{M}$ signatures found in pediatric patient HGGs and these signatures display downregulation of developmentally important Hoxa genes and upregulation of tumorigenic genes that ultimately contribute to accelerated glioma formation.

\section{H3K27M Increases Glioma Cellularity and Implicates Cell of Origin Signatures}

Histological analysis was performed to examine differences between H3K27M and H3WT tumors for common glioma cell signatures. Because pHGGs are largely heterogenous, varying in cellular composition from oligodendrocytic to astrocytic and even neuronal signatures, we used Olig2 and GFAP markers in attempt to classify mouse gliomas based on predominant cellular populations within tumors. Surprisingly, only (Brca2;p53;H3K27M) Emxl-cre tumors displayed robust Olig2 expression compared to all other gliomas, including (Lig4;p53;H3K27M) Emxl-cre (Figure 4-6A, B), suggesting $\mathrm{H} 3 \mathrm{~K} 27 \mathrm{M}$ is driving more of an effect on oligodendrocytes when combined HRdeficiency. However, expression of GFAP was significantly increased in both (Brca2;p53;H3K27M) Emx1-cre and (Lig4;p53;H3K27M) Emxl-cre tumors compared to their $\mathrm{H} 3 \mathrm{WT}$ complements, indicating H3K27M is additionally affecting astrocytic populations. To further analyze the glial cell differences observed between H3K27M and H3WT tumors we utilized GSEA and the Cahoy database again, which is specific for neural cell signatures (Figure 4-6C). This analysis revealed all H3K27M gliomas to have significant upregulation of genes similar to mature astrocytic signatures, while H3WT tumors match more closely with immature astroglial cell populations. This suggests H3WT tumors may originate from less mature progenitor cell populations than $\mathrm{H} 3 \mathrm{~K} 27 \mathrm{M}$ gliomas. 
Figure 4-6. H3K27M increases glioma cellularity and implicates cell of origin signatures.

(A) H3K27M gliomas display distinct increases in Olig2 and GFAP expression compared to H3WT tumors. (B) Quantification of total Olig2 and GFAP positive cells throughout adult mouse brain. ${ }^{*} p<0.03, * * p=0.0020, * * * * p<0.0001$. (C) GSEA identified all $\mathrm{H} 3 \mathrm{~K} 27 \mathrm{M}$ gliomas associate with more astrocytic and less astroglial signatures compared to H3WT (Cahoy database). Upregulated genes in H3K27M tumors also associated with astrocyte differentiation and affected kinase cascades through Gene Ontology for Biological Processes pathways. Length of bar indicates p-value (D) GSEA of Gene Ontology (GO) for Central Nervous System showed overlapping gene profiles with upregulated H3K27M tumor samples for neuronal development and axon guidance along with BioPlanet pathways. Length of bar indicates p-value. 


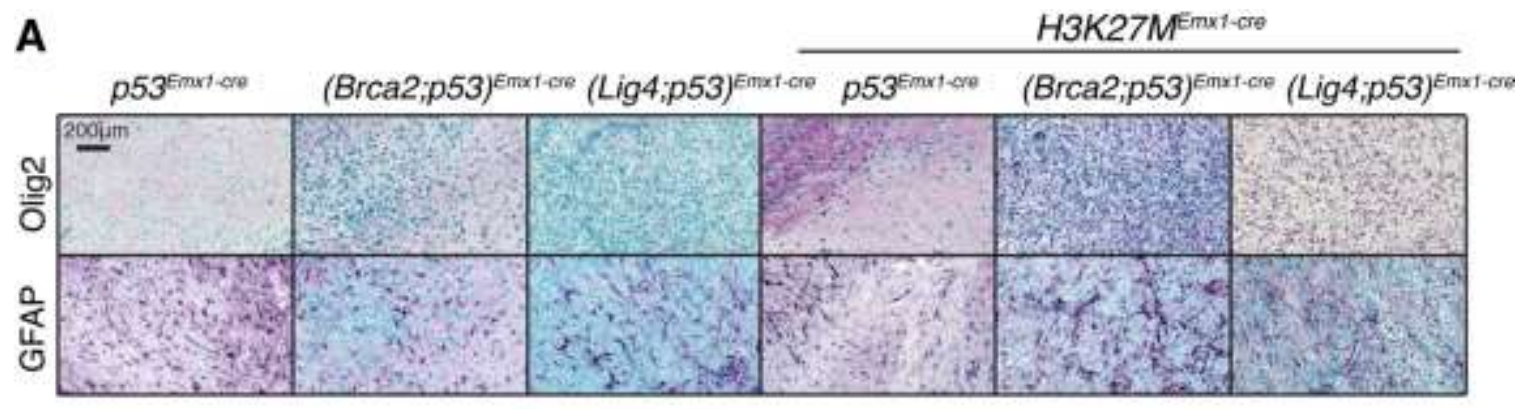

B

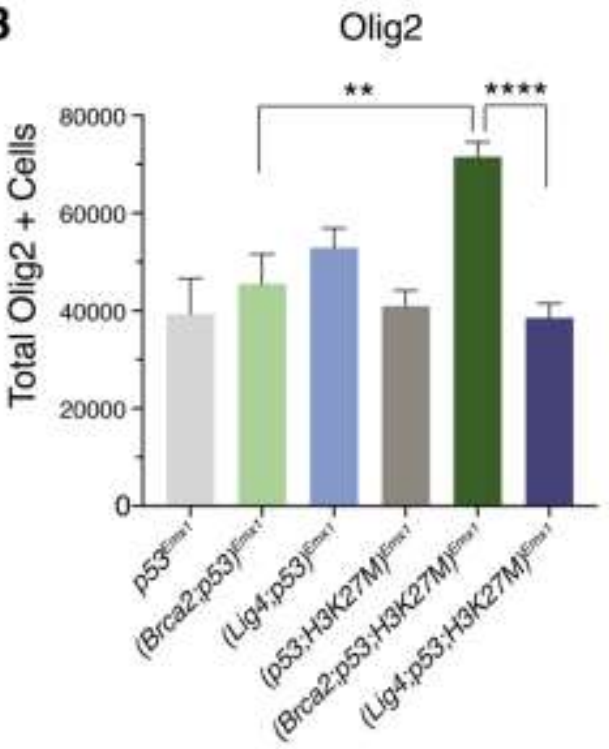

C

All H3K27M vs All H3WT
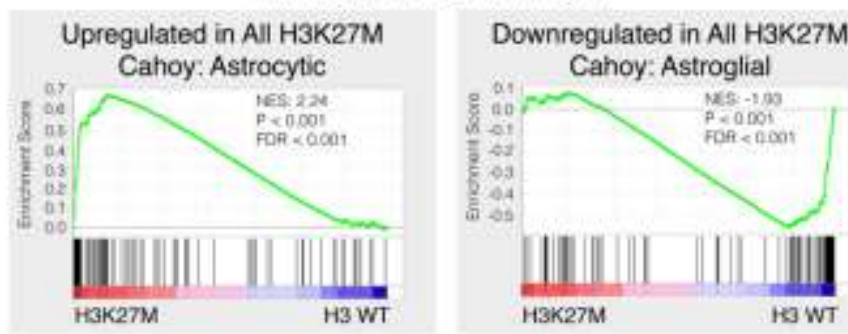

\section{Gene Ontology \\ Biological Processes}

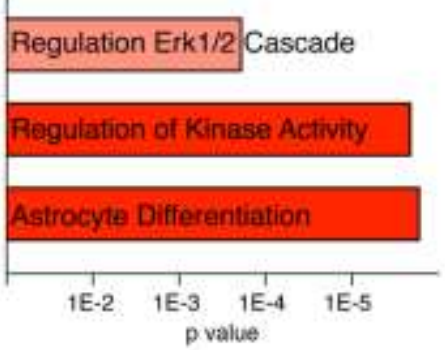

D All H3K27M vs All H3WT Upregulated in All H3K27M GO: CNS - Neuron Development

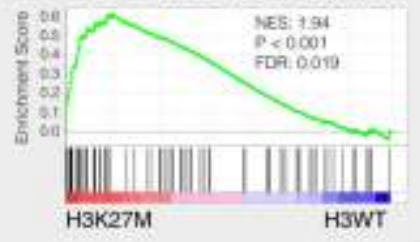

\section{BioPlanet}

Developmental Biology

Neuronal System

Axon Guidance

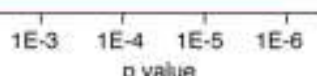


Furthermore, gene ontology pathways for biological processes uncovered similar gene association with astrocyte differentiation, primarily driven by upregulation of tumor suppressor $C d k 6$, in H3K27M tumors (Figure 4-6C) [230]. Additional upregulation of kinase cascade related genes (Egfr, Fgfr2, Ntrk2 and Alk), which are frequently altered in pediatric HGGs, were identified from same gene ontology for the H3K27M tumors [90, 103, 129]. Specifically, NTRK and $A L K$, are both tyrosine receptor kinases that have been implicated as oncogenic drivers due to their frequent chromosomal rearrangements leading to gene-fusions [231, 232]. Lastly, GSEA for gene ontology in the central nervous system and the NIH's database for human pathways (BioPlanet) identified overlapping gene enrichment in H3K27M tumors with neuronal development and axon guidance (Figure 4-6D) [233]. This suggests that H3K27M additionally effects a subset of mature neurons during gliomagenesis. Collectively, these data support that H3K27M drives similar heterogenous histopathology to human glioma cell signatures, but indicates that possible differences in cell of origins with DSBR-deficiency exist. H3K27M with loss of NHEJ implicate more mature astrocytes and neurons as targeted cells, while HRdeficiency associate a mixed glial population with a large population of oligodendrocytes also effected.

\section{H3K27M Differentiates DSBR-Deficient Gliomas by Unique Gene Expression Patterns that Effect Neural Development}

To better understand the implications of how H3K27M impacts glioma gene expression in relation to DSBR, we directly compared (Brca2;p53;H3K27M) Emxl-cre $^{\text {and }}$ (Lig4;p53;H3K27M) ${ }^{\text {Emxl-cre }}$ RNA-seq data. Unsupervised clustering of the top 331 most differentially expressed genes revealed distinct hierarchical grouping between tumor samples except for two (Lig4;p53;H3K27M) ${ }^{\text {Emxl-cre }}$ gliomas, which still appeared to share similar expression patterns with their genotypes (Figure 4-7A). Additionally, we observed an overall increase in average gene expression in (Lig4;p53;H3K27M) ${ }^{\text {Emxl-cre }}$ (data not shown), including kinases like cyclin dependent kinase 9 (Cdk9), Alk receptor tyrosine kinase, and stem-cell factor Sox9. Chromosomal rearrangements are common genetic alterations of Alk resulting multiple different fusion genes in gliomagenesis [231]. Interestingly, a well-known tumor suppressor, cyclin dependent kinase inhibitor $2 \mathrm{a}$

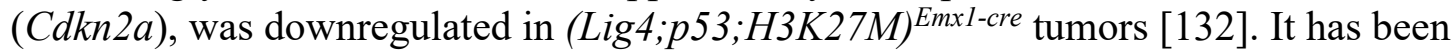
shown various $C d k n$ genes are dysregulated by the H3K27M mutation [90, 155]. GSEA using the KEGG axon guidance gene list suggests genes associated with axon guidance, like Slit and Robo, were upregulated in (Lig4;p53;H3K27M) ${ }^{\text {Emxl-cre }}$ (Figure 4-7B, C), suggesting the role these genes may play in assisting in neural cell proliferation during gliomagenesis [234]. Direct comparison of (Lig4;p53;H3K27M) ${ }^{\text {Emx1-cre }}$ to (Lig4;p53) Emx1cre, identified a subgroup of protein tyrosine phosphatase (Ptp) genes, Ptpru, Ptprn,

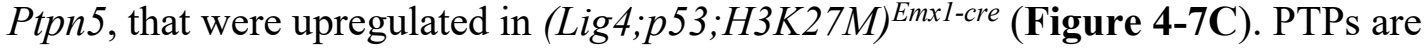
known to be involved in regulation of a variety of cellular processes including cell growth, differentiation, mitotic cycle, oncogenic transformation, and glioma biology $[129,235,236]$, indicating a tumorigenic advantage with H3K27M expression. We found upregulated genes in (Brca2;p53;H3K27M) ${ }^{\text {Emx 1-cre }}$ are associated with enriched pathways for cell differentiation and cell-matrix adhesion which may be assisting in tumor cell 
Figure 4-7. H3K27M differentiates DSBR-deficient gliomas by gene expression patterns that effect neural development.

(A) Unsupervised clustering of top 331 most differentially expressed genes between (Lig4;p53;H3K27M) ${ }^{\text {Emxl-cre }}$ and (Brca2;p53;H3K27M) ${ }^{\text {Emxl-cre }}$ tumor samples revealed distinct hierarchical grouping with increased gene expression patterns in (Lig4;p53;H3K27M) Emxl-cre . (LogFC >1.0, p<0.05, Avg. exp. >1.0). (B) GSEA shows

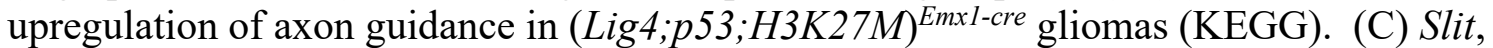
Robo and Ptp gene groups are highly upregulated in (Lig4;p53;H3K27M) ${ }^{\text {Emx } 1 \text {-cre }}$ gliomas compared to (Lig4;p53) Emx1-cre . (D) Upregulated genes in (Brca2;p53;H3K27M) EmxI-cre are associated with enriched pathways for cell differentiation and cell-matrix adhesion. BMP stimulus, DNA repair and epithelial cell proliferation were also upregulated compared to $(p 53 ; H 3 K 27 M)^{E m x l-c r e}$. (Enrichr; Gene Ontology Biological Processes). (E) Gene expression comparison of all cohorts either Lig4 or Brca2 null. Upregulation of neuron projection guidance and cell fate commitment were observed in all Lig4 ${ }^{\text {Emx } 1 \text {-cre }}$ tumors. (GSEA; Gene Ontology). (F) Synaptophysin (neural synaptic vesicle marker) is substantially reduced in (Brca2;p53;H3K27M) ${ }^{\text {Emx } 1 \text {-cre }}$ tumors and is mostly retained in Lig4-deficient samples. Graph depicts RNA-seq tumor gene expression by FPKM. IHC closeups highlight the tumor specific loss of synaptophysin. Scale bar; $1 \mathrm{~mm}$. 

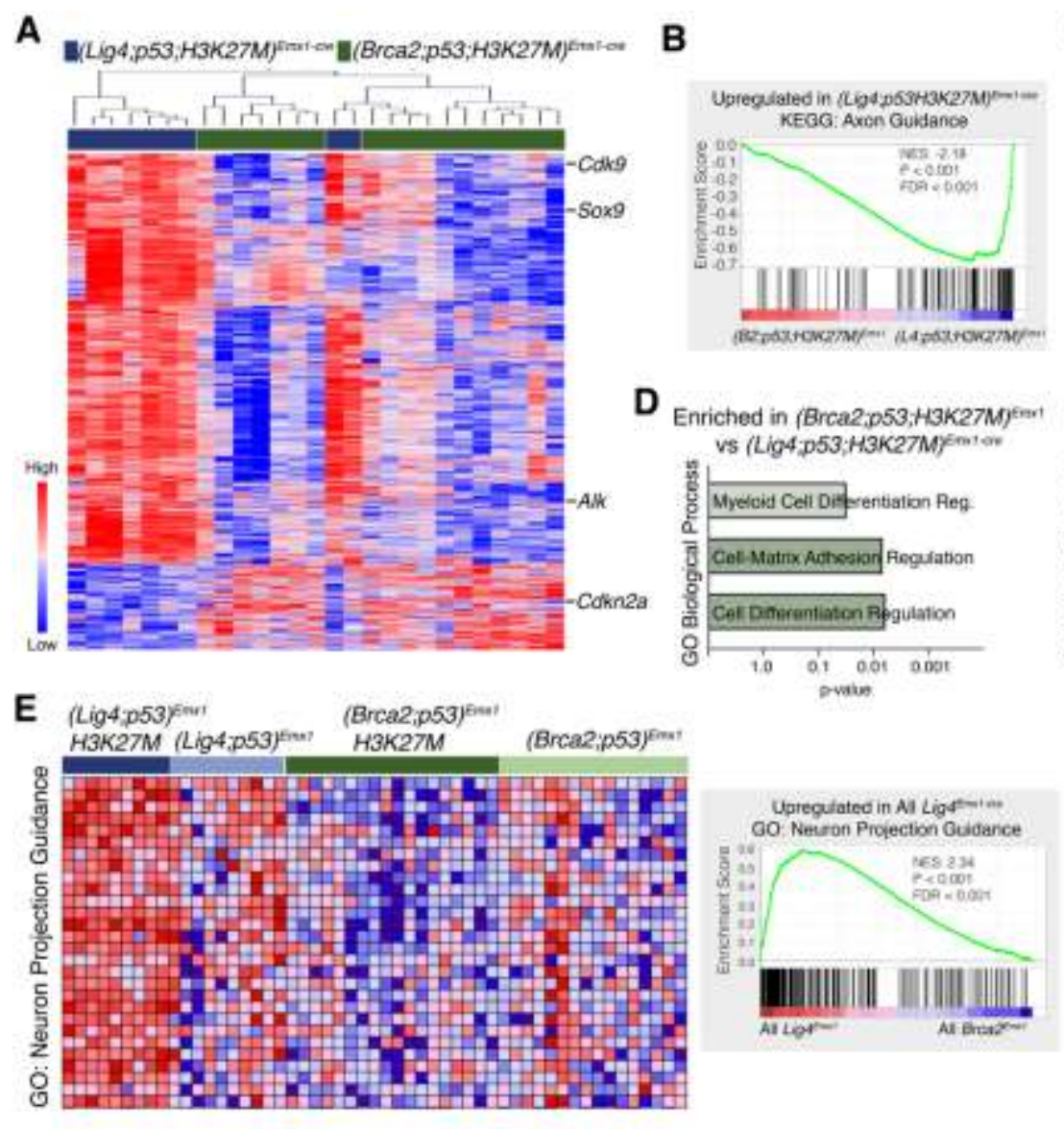

D Enriched in (Brca2;053,H3K27M) ener
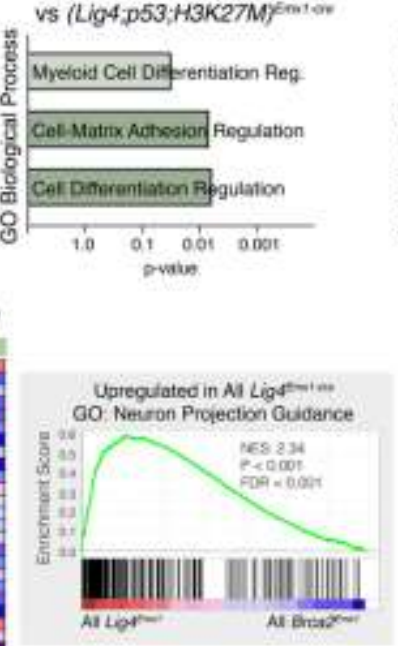

F

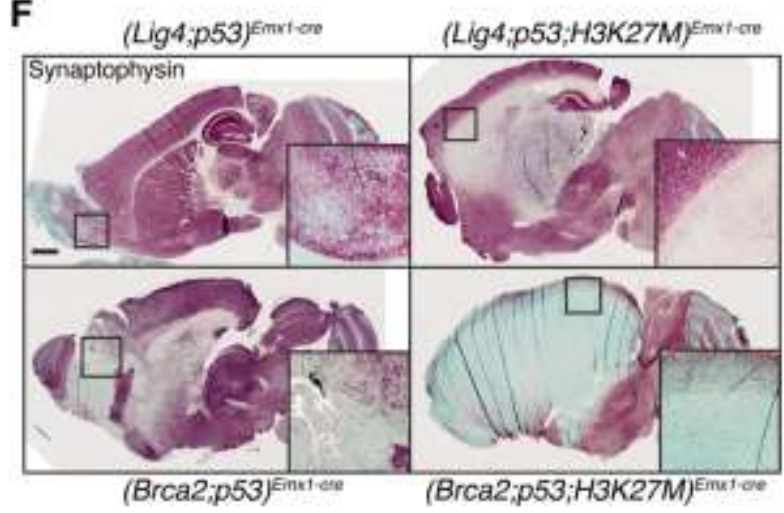

C (Lig4:p53) ${ }^{\text {Einut }}$

H3K27M Finer (Lig4,p53) Fens

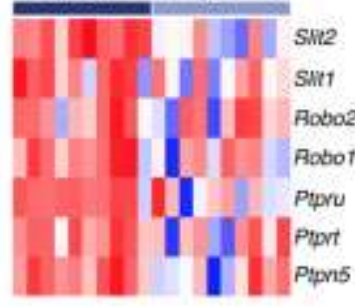

Enriched in (Brca2;p53;H3K27M) fiom vs (p53:H3K27M) finter
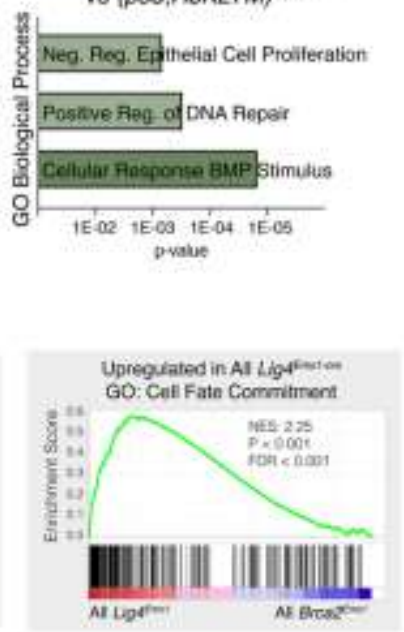

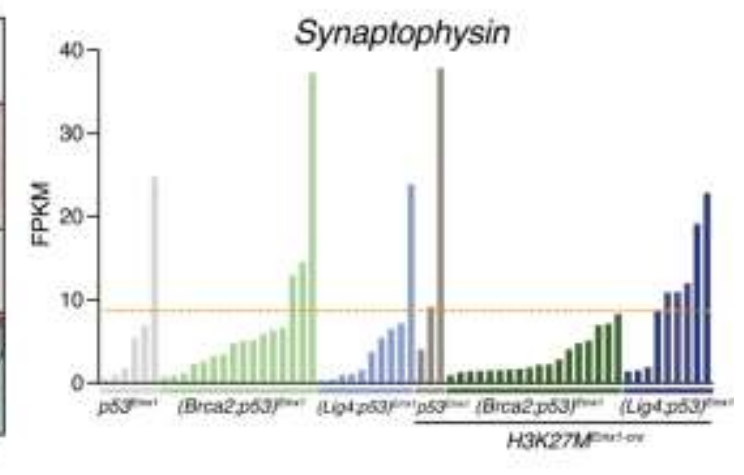


diffusion (Figure 4-7D). Gene ontology pathways revealed BMP stimulus, DNA repair and epithelial cell proliferation were also upregulated in the (Brca2;p53;H3K27M) ${ }^{\text {Emxl-cre }}$

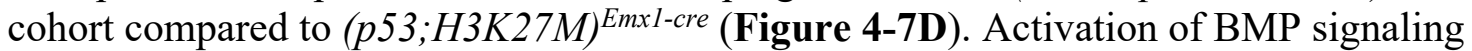
has been linked to ACVR1 (ALK2) mutations in pHGGs [90]. When comparing all gliomas of either Lig4- or Brca2-deficiency, not dependent on H3K27M, there is a clear enhanced expression pattern of neuron projection guidance and cell fate commitment genes in the Lig4-deficient tumor samples from a GSEA gene ontology dataset (Figure 4-7E). This suggests that inhibition of NHEJ may affect a more mature neuron committed cell population in glioma development. Further, expression of synaptophysin, a neural synaptic vesicle marker, displayed robust expression throughout the cortex of both (Lig4;p53) ${ }^{\text {Emx I-cre }}$ and (Brca2;p53) ${ }^{E m x 1-c r e}$ mice but was only slightly reduced in glioma tissue of (Brca2;p53) Emxl-cre (Figure 4-4F). However, a dramatic loss synaptophysin can

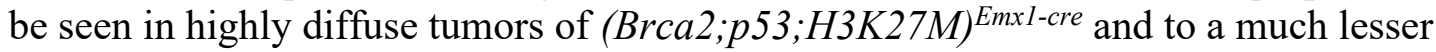
extent in (Lig4;p53;H3K27M) Emxl-cre, suggestive of a more neuronal signature when H3K27M is combined with Lig4-deficiency. RNA-seq expression levels for synaptophysin supported this. Thus, H3K27M appears to drive more dependent differential gene expression in Lig4-deficient gliomagenesis, than Brca2, with substantial upregulation of neuron specific developmental pathways, including axon and neuron projection guidance and commitment to cell fate.

\section{H3K27M Alters HGG Gene Expression and Identifies Potential Gene-Fusions}

We previously found common oncogenic gene-fusion events in HR- or NHEJdeficient mouse tumor samples, using a newly developed algorithm, CICERO [177] (Figure 3-5). Thus, we were interested in examining the effect H3K27M would have on such biological phenomena. In (Brca2;p53;H3K27M) ${ }^{\text {Emxl-cre }}$ tumors we identified a variety of gene-fusions, including Tpm3-Ntrkl (Ntrk are common fusion-genes in pHGGs), Cuxl-Tex11 (Cuxl is a haploinsufficient tumor suppressor that occasionally fuses with $F G F 1$ ), Sox6-Def8 and one involving Hmgbl (high mobility group gene involved in chromatin remodeling and DNA repair) [90, 202, 237, 238]. In addition to gene-fusions we uncovered many gene amplifications that carry potential to be genefusions in H3K27M gliomas. Two of which were growth factor receptors, EGFR and $F G F R 2$, both known to play significant roles in cancer development [126, 127, 130, 132], were amplified at rates of $75 \%(E g f r)$ and $90 \%(F g f r 2)$ in H3K27M tumors compared to $20 \%(E g f r)$ and $50 \%(F g f r 2)$ in H3WT (Figure 4-8A). Other possible amplified genefusions occurred in genes important for regulating neurogenesis and differentiation, such as Sox6 and Lin28b (Figure 4-8B). Interestingly, H3K27M tumors had decreased expression of the tumor suppressor Pten compared to H3WT and more specifically (Brca2;p53;H3K27M) ${ }^{\text {Emxl-cre }}$ gliomas had significant loss of the proto-oncogene Kras (Figure 4-8C), suggesting the involvement of other key drivers of tumorigenesis. Another unique finding within the $(B r c a 2 ; p 53 ; H 3 K 27 M)^{E m x l-c r e}$ tumors was that they carried about half the amount of average somatic mutations, determined by whole exome sequencing, as the counterpart tumors without H3K27M (Figure 4-8D), indicative of both the decreased tumor free survival age of these mice and that H3K27M pHGGs generally possess fewer overall mutations. (Brca2;p53;H3K27M) ${ }^{\text {Emxl-cre }}$ mice succumbed 
A
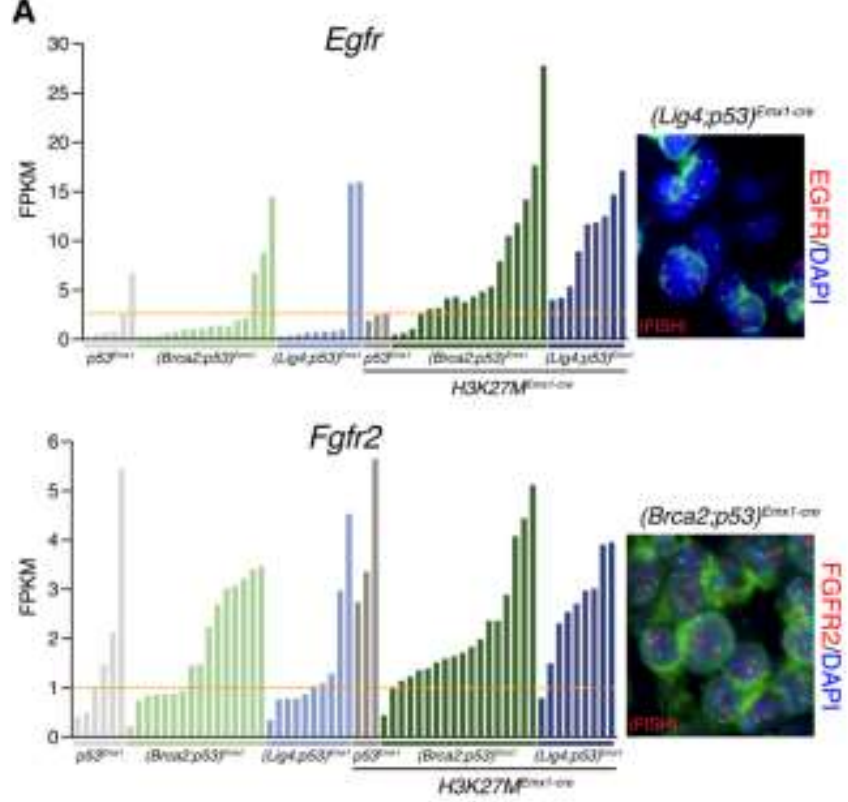

C
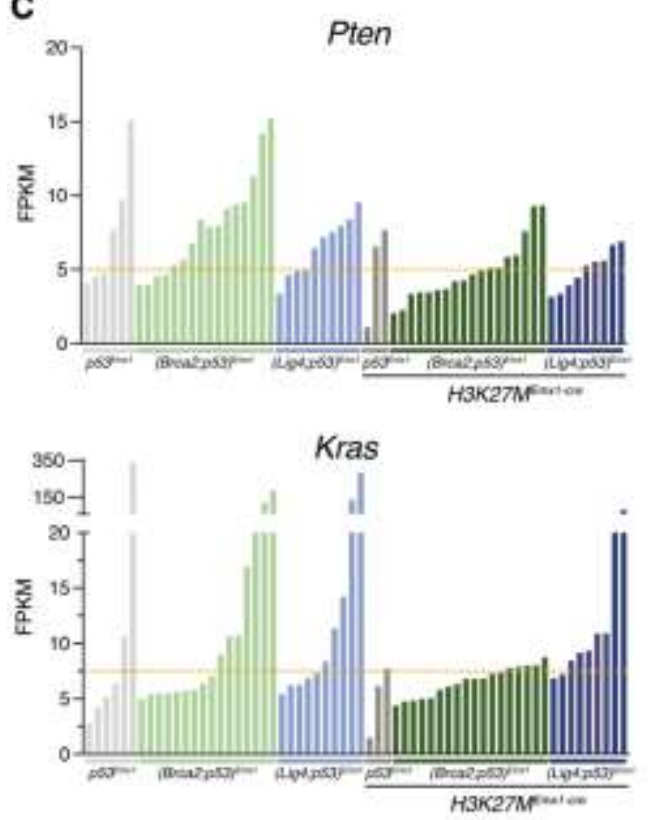

B
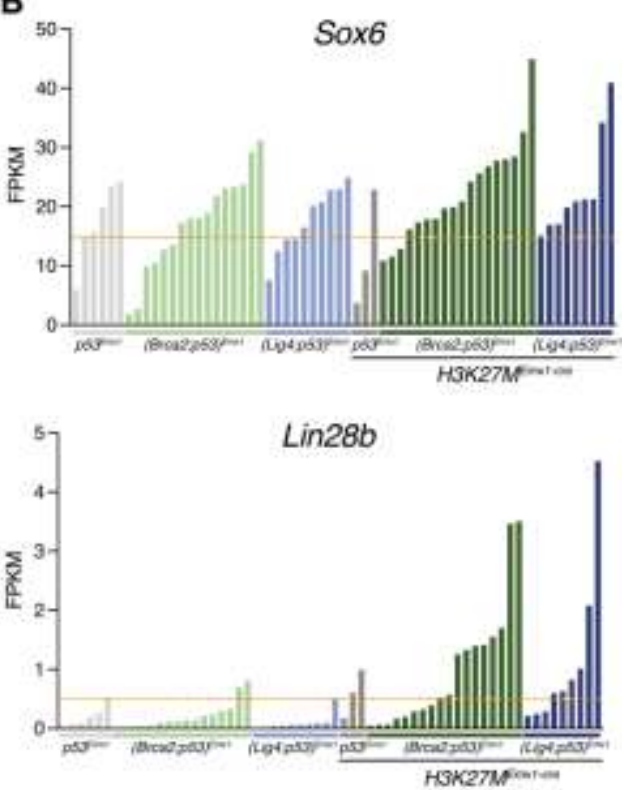

D Somatic Mutations

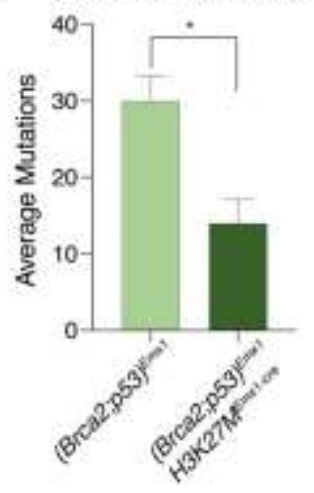

Figure 4-8. H3K27M alters HGG gene expression causing potential gene-fusions. (A) Potential gene-fusions Egfr and Fgfr2 have higher expressions when H3K27M is present in gliomas. FISH analysis confirmed cellular expression. (EGFR - red, chromosome 11 control probe - green and FGFR2 - red, chromosome 7 control probe green). (B) Sox6 and Lin28b display gene amplifications in H3K27M gliomas. (C) Pten and Kras expression are decreased in H3K27M tumors. Kras is specifically reduced in (Brca2;p53;H3K27M) ${ }^{\text {Emxl-cre }}$. (D) (Brca2;p53) Emxl-cre $(\mathrm{n}=7)$ tumors had more overall somatic mutations on average than $(B r c a 2 ; p 53 ; H 3 K 27 M)^{\text {Emxl-cre }}(\mathrm{n}=7)$ tumors from whole exome sequencing $(>5$ reads per mutant and variant allele frequency $>0.1, p$-Value $=0.0111$ ). 
to gliomas on average two months more quickly than (Brca2;p53) Emxl-cre mice (Figure 4-2A), allowing less time for somatic mutations to accumulate in the H3K27M group. These data reveal that $\mathrm{H} 3 \mathrm{~K} 27 \mathrm{M}$ contributes to altering expression of genes specific to assisting in accelerating tumorigenesis, including gene-fusions and additional oncogenes.

\section{H3K27M Increases Expression of Bivalent Gene Promoters in HGGs}

The epigenetic modification $\mathrm{H} 3 \mathrm{~K} 27 \mathrm{me} 3$, as previously described, is generally associated with repressed loci, while $\mathrm{H} 3 \mathrm{~K} 4 \mathrm{me} 3$ is primarily found at active promoter sites [166, 239]. The presence of both H3K27me3 and H3K4me3 define a potential poised or bivalent gene promotor [166]. When the H3K27M mutation is expressed, loss of $\mathrm{H} 3 \mathrm{~K} 27 \mathrm{me} 3$ leaves only $\mathrm{H} 3 \mathrm{~K} 4 \mathrm{me} 3$ left to drive gene promoter activation resulting in overall higher gene expression [160, 239]. We directly compared our list of significantly up- and downregulated genes in the absence or presence of the H3K27M mutation from tumor samples with a gene list generated by Suzanne Baker's group [160] and identified a similar proportion of potential bivalent genes when $\mathrm{H} 3 \mathrm{~K} 27 \mathrm{M}$ is present (Figure 4-9A). There was an approximately $30 \%$ increase in bivalent upregulated genes and $28 \%$ increase in downregulated genes in $\mathrm{H} 3 \mathrm{~K} 27 \mathrm{M}$ mouse tumors compared to the total gene list of $\sim 12 \%$ bivalent genes (Figure 4-9A). Of the upregulated bivalent genes, Enrichr analysis identified Gene Ontology Biological Processes to be upregulated, specifically pathways highly associated with kinase activity, epithelial cell proliferation and Wnt signaling (Figure 4-9B). Particular genes driving the upregulation of these pathways include some that we identified previously and a couple novel ones: Sox9, Ntrk1, Egfr, Ptpru, Cdk6, Wnt3 (proto-oncogene) and Cdkn1c (tumor suppressor). Closer examination of direct comparisons of H3K27M groups with or without DSBR inhibition indicates $(p 53 ; H 3 K 27 M)^{E m x 1-c r e}$ gliomas had a much larger percentage of upregulated bivalent genes compared to $p 53^{\text {Emxl-cre }}$ control tumors, which is reflective of what the Baker lab model uncovered. However, the greatest difference in bivalent gene promoters was evident when we compared Brca2- and Lig4-deficient tumors (Figure 4-9C). (Lig4;p53;H3K27M) ${ }^{\text {Emx l-cre }}$ gliomas had almost 4 times the amount of upregulated bivalent genes compared to (Brca2;p53;H3K27M) ${ }^{\text {Emxl-cre }}$. Surprisingly, (Brca2;p53;H3K27M) ${ }^{\text {Emxl-cre }}$ actually had a larger percentage of bivalent genes considered downregulated. The difference between DSBR-deficient tumors with H3K27M may be due to different effected neural cell populations. In the absence of the histone mutation, H3WT gliomas, displayed a substantial increase in the proportion of downregulated bivalent gene promoters (Figure 4-9D). Although (Brca2;p53) Emxl-cre $^{-}$ tumors had more overall genes marked with $\mathrm{H} 3 \mathrm{~K} 27 \mathrm{me} 3$ and $\mathrm{H} 3 \mathrm{~K} 4 \mathrm{me} 3$ compared to

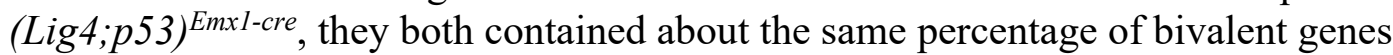
throughout. These data support that bivalency release via $\mathrm{H} 3 \mathrm{~K} 27 \mathrm{me} 3$ reduction is a highly probable mechanism for how H3K27M facilitates differential gene expression signatures that drive gliomagenesis.

With the development and advancements in single-cell RNA-sequencing much of the mouse brain cell network has been mapped to known gene markers [240]. Utilizing an established dataset from Rosenberg et al. [240], we compared the mouse glioma RNA- 
Figure 4-9. H3K27M increases bivalent gene promoters in HGGs.

(A) Stacked bar graphs showing promoter status for genes up- or downregulated in H3K27M HGGs. Gold bars represent proportion of genes with observed bivalent promoters (H3K27me3+ H3K4me3+) from DIPG (Larson et al.) dataset that are upregulated in the H3K27M mutant HGGs compared to H3WT. (B) Gene ontology enrichment analysis for upregulated genes in $\mathrm{H} 3 \mathrm{~K} 27 \mathrm{M}$ gliomas that display bivalent promoters, as shown in (A) right panel, identifies effected pathways. Length of bar indicates p-value. (C) Comparisons of all groups with up- or downregulation of bivalent genes with the H3K27M mutation present. Total number of genes are shown on left with greatest increase in upregulated in (Lig4;p53;H3K27M) Emxl-cre. Right graph shows the percentage change in bivalent genes for all groups for up- or downregulated genes. (D) Bivalent gene graphs highlighting all groups with H3WT only. Full gene counts (left) and percentages (right). (E) Glioma grouping by average expression levels of Slit1,2,3 genes and enrichment in gene sets corresponding to different cell populations along with diffusion and genotype showing correlation between Slit expression, cortical cell enrichment and diffusion (Rosenberg et al.). 
A

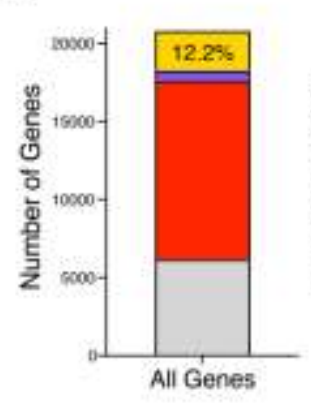

All H3K27M ys All H3WT

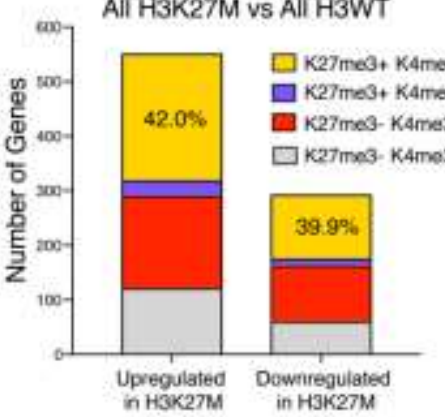

B

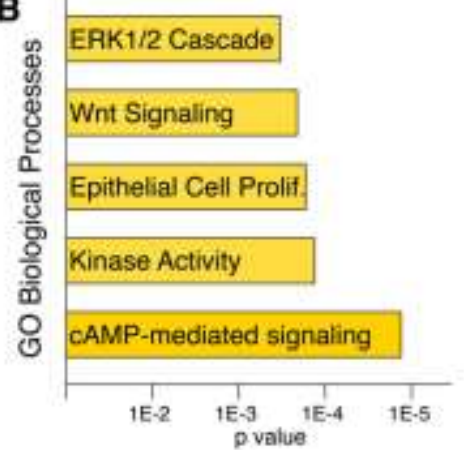

C

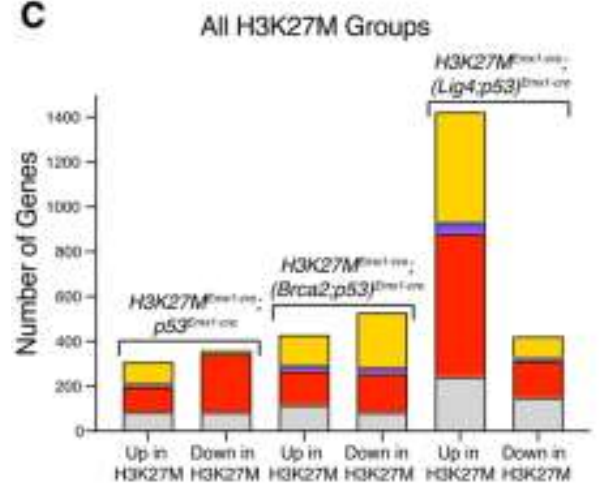

D

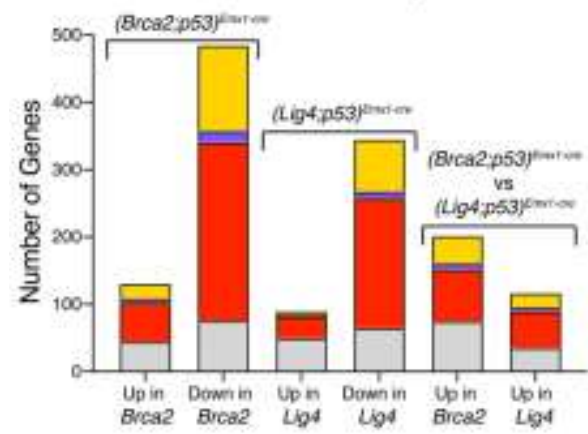

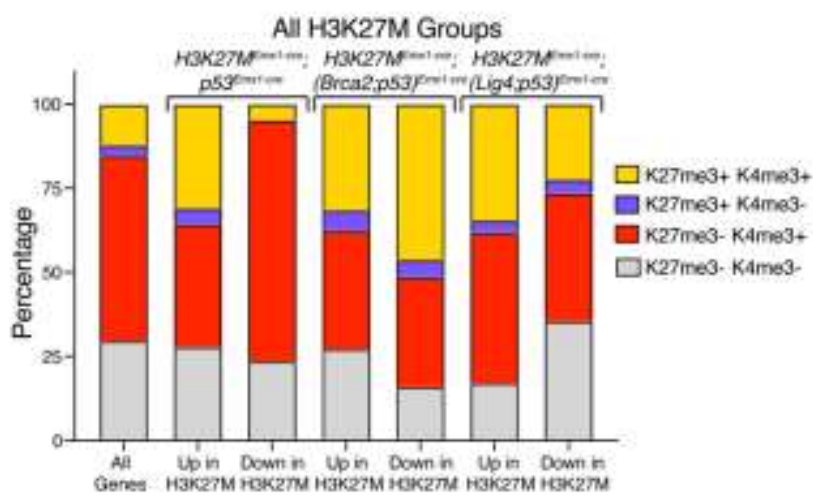

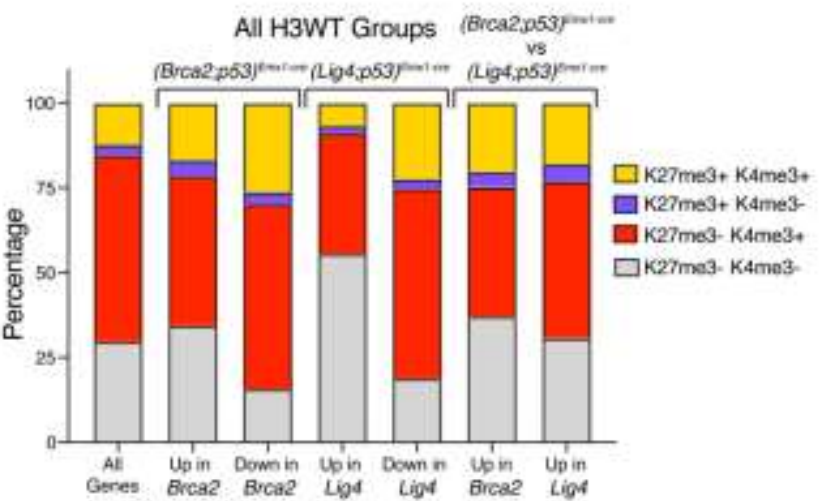

\section{E}

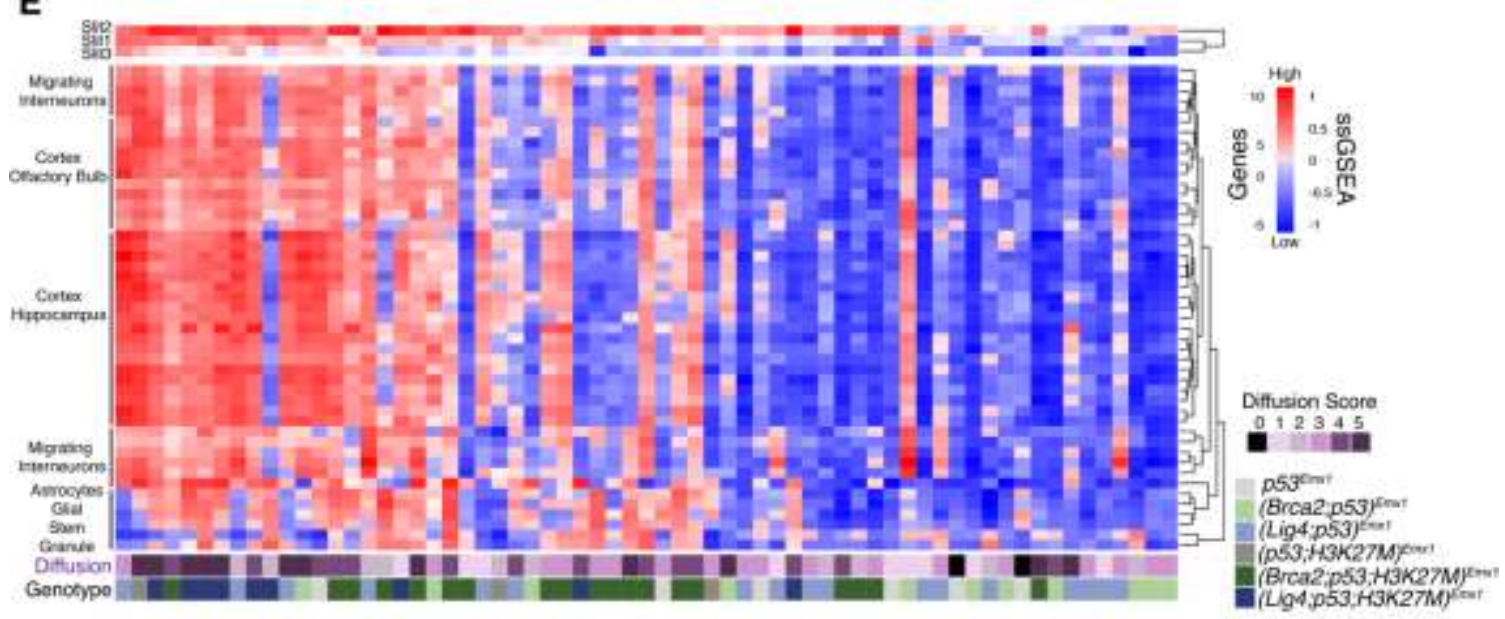


seq data to gene expression patterns to specific brain cell populations. Based on our previous findings identifying a strong differential gene expression pattern of important neuron development genes (Slit-Robo pathway) between Lig4- and Brca2-deficient tumors (Figure 4-7B, C), we decided to additionally sort by Slit1/2/3 genes to examine possible correlations with other neuron cell populations. We were not only able to distinguish cell populations by their genotype group but also associate overall tumor diffusion to these clusters (Figure 4-9E). A majority of mouse tumors with H3K27M have higher gene expression in the brain regions of the cortex, olfactory bulb, hippocampus and some migrating interneuron populations. (Lig4;p53;H3K27M) ${ }^{\text {Emxl-cre }}$ gliomas displayed the greatest gene expression in these regions with corresponding high diffusion scores (Figure 4-9E, far left side). Increased tumor diffusion in H3K27M groups, previously determined by histology (Figure 4-2C), correlated with these neural gene expression patterns with similar regions of the brain effected, including dispersed tumor cells throughout the cortices, olfactory bulbs and hippocampus. These results suggest that the addition of the H3K27M mutation with concurrent loss of either NHEJ or HR, severely disrupt the overall tumor genomic expression profile in the mouse cortex and other neural cell populations, however this disruption is to a much greater extent with NHEJ-deficiency.

\section{H3K27M Determines Cell of Origin Susceptibility to Gliomagenesis}

The importance of histone marks, H3K4me3 and/or H3K27me3, at specific stages of neural lineage progression are known to have direct effects on neural development $[106,166]$. We examined the link between the specific cell of origin and histone marks at critical genes that may be responsible glioma formation in our model. Principalcomponent analysis (PCA) indicates high heterogeneity presence when comparing all mouse tumor samples to each other (Figure 4-10A). Interestingly, (Brca2;p53;H3K27M) $)^{\text {Emxl-cre }}$ and (Lig4;p53;H3K27M) ${ }^{\text {Emxl-cre }}$ are the only two genotypes that exhibit unique grouping from each other in the three-dimensional PCA (red and blue circles), highlighting the significant role that $\mathrm{H} 3 \mathrm{~K} 27 \mathrm{M}$ plays in differential tumor development when combined with HR- or NHEJ-deficiency. We compared the glioma RNA-seq data directly to methylation marks determined by Wieland Huttner's group [106] and found genotypes separated into unique groups of differentially expressed genes as shown in the heatmap (Figure 4-10B). A particular subset of these genes (outlined by red box) are universally upregulated by $\mathrm{H} 3 \mathrm{~K} 27 \mathrm{M}$, therefore have a similar genome-wide effect on tumor development. These include but are not limited to Sox 1 (embryonic development), Egfrl (commonly mutated cancer gene), Eya4 (DNA repair), and Car8 (cerebellar ataxia). More importantly another subgroup of genes, identified from the methylation dataset, uniquely separated the effect that loss of HR or NHEJ had on tumors with the H3K27M mutation (outlined by black box) (Figure 4-10B). Distinctive genes

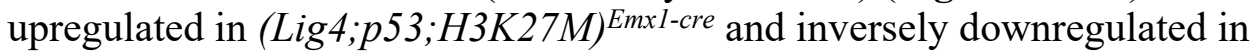
(Brca2;p53;H3K27M) ${ }^{\text {Emxl-cre }}$ tumors include Ntn1 (axon guidance and cell migration during development), Adra2a (nervous system development), $\operatorname{Vg} f$ (nerve growth factor inducible) and interestingly $C b x 7$, which is part of the PRC1 complex with its primary function being repression of $C d k n 2 a$. Further comparison of the triple mutant tumor 
Figure 4-10. Addition of H3K27M determines cell of origin susceptibility to gliomagenesis between Lig4 and Brca2 loss.

(A) 3D PCA plot comparing all genotypes to each other. (Lig4;p53;H3K27M) Emx1-cre (blue circle) and (Brca2;p53;H3K27M) ${ }^{\text {Emxl-cre (red circle) highlighted to indicate greatest }}$ difference between subgroups. All other groups show the large tumor heterogeneity of gliomas. (B) Heatmap from the top 100 sorted genes which exhibit methylation marks during neocortical development. Similar upregulated genes for H3K27M samples are highlighted in red box. Differential gene expression for (Lig4;p53;H3K27M) ${ }^{\text {Emxl-cre }}$ and (Brca2;p53;H3K27M) Emxl-cre is highlighted in the black box. (C) GSEA comparing

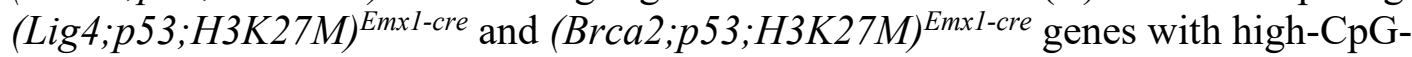
density promoters (HCP) bearing the tri-methylation mark $\mathrm{H} 3 \mathrm{~K} 27 \mathrm{me} 3$ in MCV6 cells. Genes with HCP bearing H3K4me2 and H3K27me3 in neural precursor cells (NPC). (D) The most upregulated genes in (Lig4;p53;H3K27M) ${ }^{\text {Emxl-cre }}$ or (Brca2;p53;H3K27M) $)^{\text {Emxl- }}$ cre indicate differences related to cell type and cortical location of primary DNA damage accumulation $(\gamma \mathrm{H} 2 \mathrm{AX})$ in early development at E15.5. Lig4 - upper cortex more differentiated/mature neurons. Brca2 - deep cortex more progenitor-like. Genes previously identified to be bivalent in mouse NECs (Huttner et al., 2017; gold) were compared to our dataset to identify the percentage of genes marked either H3K4me3 (red), H3K27me3 (purple), bivalent (gold), or no modifications (gray), in the defined neural cell populations, aRG-N or Neurons, during neural lineage progression. Genes relate directly to the bivalency at NEC for aRG-N and Neuron comparisons ( $\operatorname{LogFC}>1.0$ and $\mathrm{p}<0.05$ ). (E) The total number of genes marked for each methylation state and cell type, as shown in (D), is represented. (Lig4;p53;H3K27M) ${ }^{\text {Emx } 1 \text {-cre }}$ have substantially more upregulated genes in each methylation state compared to (Brca2;p53;H3K27M) ${ }^{\text {Emx I-cre }}$. Example genes present in the three different histone marks are given below each graph. *indicates present in both cell types. 

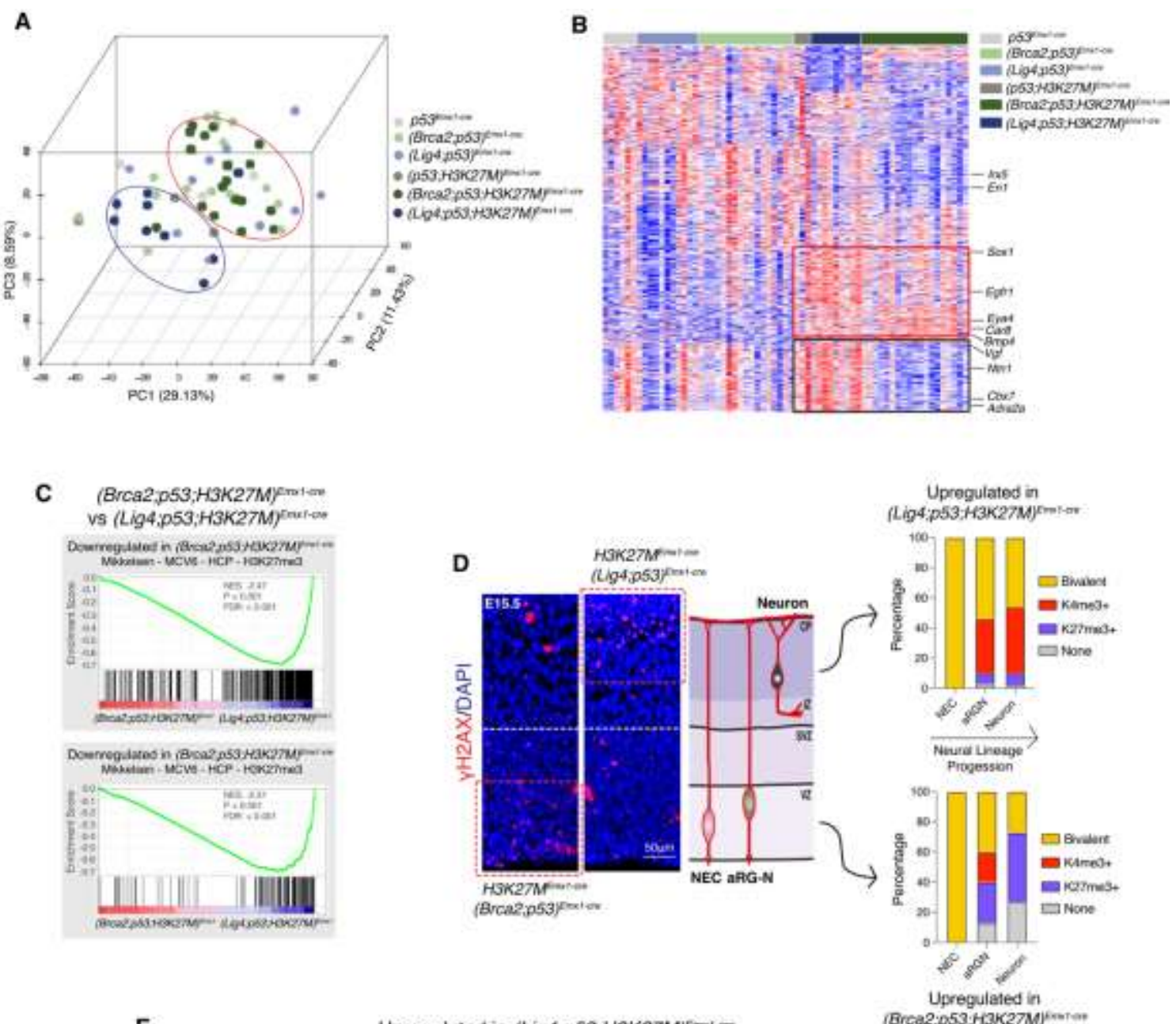

E

Upregulated in (Lig4; $53 ; H 3 K 27 M)^{5-m a t-o n}$ Brca2.p53:H3K27Mj):m-
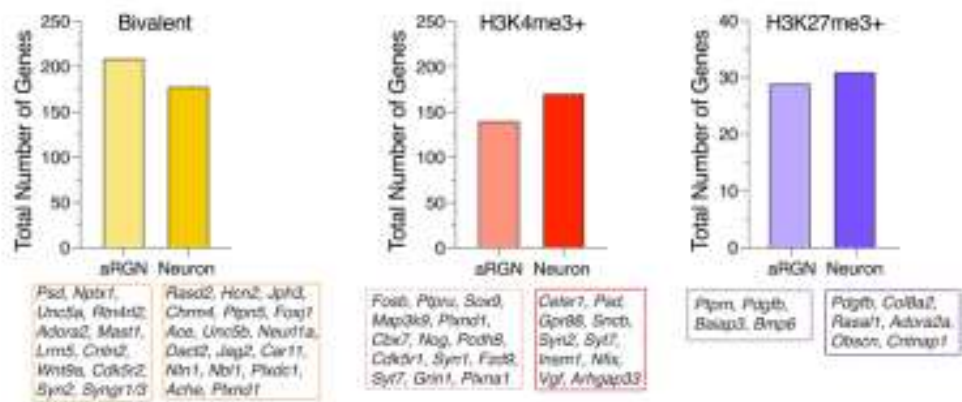

Upregulated in (8rCa2;p53,H3K27MF fartere
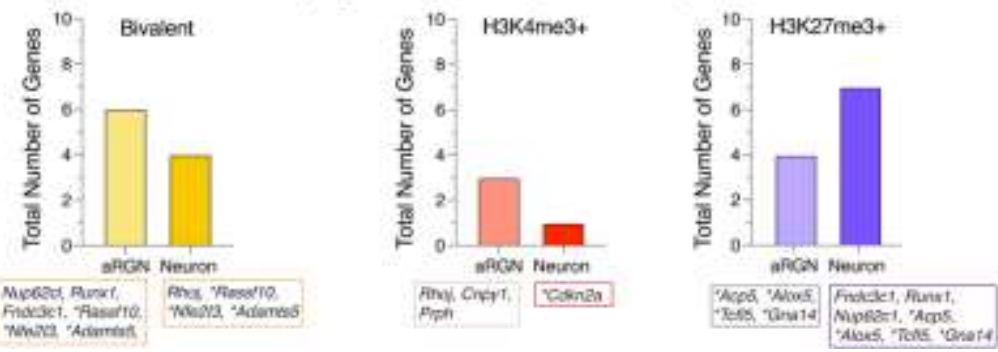
groups to each other by GSEA gene sets for hallmark pathways from the Molecular Signatures Database demonstrates differences within high-CpG-density promoters (HCP) bearing the tri-methylation mark H3K27me3 in MCV6 cells, which Mikkelsen et al. [241] have shown to be unable to fully re-program leaving cells in a more lineage committed differentiated state (Figure 4-10C). (Lig4;p53;H3K27M) ${ }^{\text {Emx I-cre tumors }}$ displayed strong enrichment within the MCV6 cells comparison, indicating a release of more genes from a bivalent state with less $\mathrm{H} 3 \mathrm{~K} 27 \mathrm{me} 3$ mark present. Additionally, we used GSEA to compare our datasets to a HCP gene set bearing H3K4me2 and H3K27me3 in neural precursor cells (NPC), which are known to have bivalent promoters [242]. We found a similar robust enrichment in the (Lig4;p53;H3K27M) ${ }^{\text {Emxl-cre }}$ group, which is consistent with a more differentiated underlying state. Comparatively, (Brca2;p53;H3K27M) ${ }^{\text {Emxl-cre }}$ does not show enrichment in these gene sets.

As previously shown, the greatest difference in accumulation of DNA damage early in development at E15.5 was between the location within the cortex of (Brca2;p53;H3K27M) ${ }^{\text {Emxl-cre (deep layers) and (Lig4;p53;H3K27M) }}$ EmxI-cre (upper layers) embryos (Figure 4-10D, left panels). This highlights the potentially different progenitor cell populations effected by Brca2- and Lig4-deficiency in these cortical layers, with Brca 2 more likely effecting early born stem/neuroepithelial cells (NECs) or apical progenitor radial glia committed to the neurogenic lineage (aRG-N) as described by Albert et al. [106]. While Lig4 may have more of an effect on differentiated/mature neural cells that are post-mitotic in the upper neocortical layers. Genes that were previously identified to be bivalent in mouse NECs [106] (gold) were compared to the $\mathrm{H} 3 \mathrm{~K} 27 \mathrm{M}$ tumor data to analyze the percentage of H3K4me3 (red), H3K27me3 (purple), bivalent (gold), or no modifications (gray), in the defined neural cell populations (aRG-N and Neuron) (Figure 4-10D, right graphs). A large majority of the most upregulated

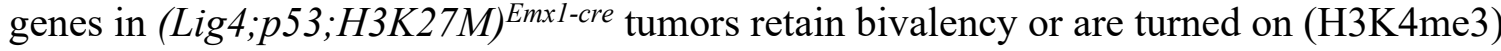
as cells mature from NECs to neurons (Figure 4-10E, upper graphs). In contrast, the genes that are upregulated in the $(B r c a 2 ; p 53 ; H 3 K 27 M)^{\text {Emxl-cre }}$ group that were bivalent in NECs were greatly reduced in total gene number in both aRG-N and neurons. However, these same genes display a higher percentage that are turned off or silenced via H3K27me3 alone through maturation (Figure 4-10E, lower graphs). Not surprisingly both groups displayed reduced gene promoters with the H3K27me3 mark, validating the effect of the H3K27M mutation. Affected genes linked to tumorigenesis and neurodevelopment include $P d g f \beta, C d k n 2 a$, Sox 9 , and Syn2 (Synapsin II) in the (Lig4;p53;H3K27M) Emxl-cre group and Runxl (also known as oncogene AML1) and Alox5 (regulator of leukemia stem cells) in the (Brca2;p53;H3K27M) ${ }^{\text {Emx I-cre }}$ tumors. Interestingly $C d k n 2 a$ was the only gene positive for $\mathrm{H} 3 \mathrm{~K} 4 \mathrm{me} 3$ through all of the

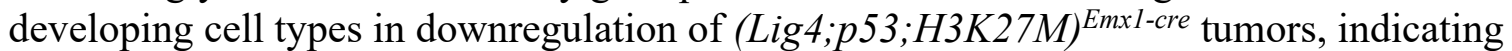
its active gene expression and underlining its importance in gliomagenesis for this particular group. Collectively, these data show in the mouse neocortex the presence of the H3K27M mutation and Lig4-deficiency, results in DNA damage that preferentially effects a more mature neural cell population and genes in these cells are critical for proper neural development and tumorigenesis. Nevertheless, Brca2-deficiency with $\mathrm{H} 3 \mathrm{~K} 27 \mathrm{M}$ cause DNA damage primarily in proliferating and immature progenitors, 
therefore reprogramming and altering the pluripotency potential by decreasing bivalency within these critical early born progenitor cells that eventually transform into tumor cells. 


\section{CHAPTER 5. DISCUSSION}

This dissertation analyzes and summarizes the role of DSBR, HR (Brca2deficiency) or NHEJ (Lig4-deficiency), during neurogenesis and high-grade glioma development. Mutations in DNA repair genes have been linked to various neurodegenerative diseases and cancers, including brain tumors [66, 243-245]. These diseases underly the importance that DNA repair pathways play in maintaining genome stability during early development of the nervous system. However, much is still unknown regarding the pathobiology of defective DNA repair including DSBR, particularly in the context of specific neural cell types and regions of the brain. Therefore, understanding how gene alterations in early brain development result in tumorigenesis will help us answer these questions. Throughout this dissertation I have presented my work investigating how the loss of DSBR, HR (Brca2) or NHEJ (Lig4), effects neurogenesis and eventually leads to gliomagenesis in the mouse cortex. Additionally, I examined a histone $\mathrm{H} 3(\mathrm{H} 3 \mathrm{~K} 27 \mathrm{M})$ mutation that influences high-grade gliomagenesis, to identify potential cooperation between this oncohistone and heightened genome instability.

\section{DSBR-Deficiency in Cortical Progenitor Cells}

Analysis of the loss of DSBR, HR and NHEJ via conditional Brca2 and Lig4 loss, respectively, using Emxl-cre recombinase to target gene inactivation to the neocortex revealed that Brca2 $2^{\text {EmxI-cre }}$ and Lig4 $4^{\text {Emxl-cre }}$ resulted in DNA damage accumulation that correlates with apoptosis in specific layers of the neocortex (Figure 3-1). Similarly, DSBR mutant mice with additional $p 53$ inhibition revealed large amounts of DNA damage present in the neocortex, however more in the form of DSBs identified by $\gamma \mathrm{H} 2 \mathrm{AX}$-foci. (Brca2;p53) Emxl-cre embryos at E12.5 had more damage accumulation, but just three days later in development at E15.5 (Lig4;p53)EmxI-cre embryos amassed significantly more DNA damage, especially in the upper post-mitotic cortical layers (Figure 3-6). Contrastingly, (Brca2;p53) ${ }^{\text {Emx } x \text {-cre }}$ cortices primarily retained damage to the deep proliferative layers, supporting the differential roles HR and NHEJ play in DSBR in specific cell populations of the developing cortex. The difference in DNA damage present at E12.5 between Brca2-and Lig4-deficient mice highlights the importance and efficiency of HR in early proliferative progenitors. In the progenitors where HR is inhibited, the cells rely on NHEJ, which as a consequence of its error-prone nature occasionally results in improperly repaired DNA. When NHEJ is inhibited at E12.5, we see less DNA damage accumulation compared to HR-deficiency, albeit still at a high level compared to controls. This is because HR cannot completely compensate for the loss of NHEJ, which is more functional throughout the cell cycle. Brca2 $2^{\text {Emx I-cre }}$ primarily affected the "deep" VZ and SVZ cortical layers, while Lig4 ${ }^{\text {EmxI-cre }}$ largely created cell death in the "upper" $C P$ and MZ layers. This clear preferential difference in affected cortical cell layers between Brca2-and Lig4-deficient mice was frequently identified throughout my studies, supporting the importance of HR in replicating cortical progenitor cells and NHEJ in post-mitotic neurons. 
A specific subset of the earliest born stem and progenitor cells reside at the apical surface of the neocortical VZ, referred to as apical stem or progenitors (APs). APs undergoing mitosis were identified using the phospho-histone $\mathrm{H} 3$ mitotic marker, which allowed us to confirm activation of the G2/M checkpoint by DNA damage in these cells.

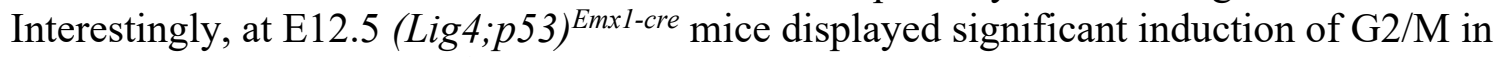
APs, while (Brca2;p53) Emxl-cre indicated no change in mitotic positive APs compared to controls. However, at E15.5 both DSBR mutants activated the G2/M checkpoint to similar extents. These results validated that DNA damage induced the G2/M cell cycle checkpoint in the critically important AP cell population of the cortex. It's been shown that cells in which p53 is inhibited, like in our model, lose the G1/S checkpoint and therefore cannot arrest at the G1/S transition [204], although they can maintain a G2/M arrest. For this reason, we did not see any significant activation of the G1/S checkpoint in any of the mice through BrdU analysis. Though this is by no means a robust study on cell cycle dynamics it is intriguing to speculate on the differential consequences that loss of HR and NHEJ have on the cell cycle of cortical progenitors in early development. From E10 to E12, rapidly proliferating APs undergo symmetric divisions and have about 3-4 times longer S-phases than after the cells differentiate [24]. However, after E13, differentiated cells have shorter S-phases and longer G1 phases [25]. We know that genome maintenance is critical for early-born APs and the increased S-phase length allows for cells to invest more time into quality control [21,24]. This is a stage in which BRCA2 and HR function is essential for maintaining DNA integrity during replication. Because we observed HR-deficient embryos having relatively higher amounts of DNA damage accumulation at E12.5, than NHEJ, this suggests BRCA2 and HR may have increased importance in early progenitors because of the longer S-phase. Additionally, previous data from our lab has shown the possibility of different damage threshold limitations in the earlier born progenitors. We showed that cortical progenitors have increased sensitivity to DNA damage inducing agents at E11 compared to E14 [75]. Thus, the loss of HR during the longer and more critical genome surveillance period, in early-born cortical progenitors, compromises DNA fidelity by the accumulation of stalled replication forks and DSBs which allow for increased gene alterations and mutational burden.

The complex events associated with cell cycle regulation and DNA damage threshold may offer an explanation to why neither mice using Nestin-cre, (Brca2;p53) Nestin-cre or (Lig4;p53) Nestin-cre, develop HGGs but rather form medulloblastomas [49, 58, 169]. Perhaps this is also due to differences in transcriptional programing of cortical progenitors at the stages of Emx l-and Nestin-cre expression. Although the activation of either cre is only separated by about one embryonic day, with Emx1-cre earlier at $\sim \mathrm{E} 9.5$, this is enough time for progenitor cells in the dorsal telencephalon to undergo significant expansion and cell fate commitment to corticogenesis [74]. In a similar manner, Nestin-cre targets progenitors that more committed to other cell fates like cerebellum development, including a large population of granule neurons in the hindbrain, which by probability increase the likelihood of medulloblastoma formation. It's also possible that the Nestin-cre mouse used was insufficient for recombination in early neural progenitors, as one group discovered [61]. This is not to say that Emxl-cre expression does not have off target effects. Aside from 
mice succumbing to HGGs, many are prematurely euthanized for various reasons including clavicle masses or submandibular cervical lymph node enlargements, indicating possible Emxl-cre nonspecific effects [246, 247]. Consequently, it appears that as progenitors progress through lineage specificity, the cells intrinsic properties may change concerning their susceptibility to DNA damage, cell cycle dynamics and principal replication differences. A previous study by our lab supports this concept as it discovered that TopBP1, a protein linked to DNA replication, prevents replication-associated DNA strand breaks in early cortical progenitors in an Emxl-cre mouse model but not in Nestincre mice [75]. Studies like these, using genetically engineered mouse models allows us to speculate the differences in regulation of DDR as progenitors mature. Therefore, we propose BRCA2 and LIG4 contribute uniquely to genome stability in early-born and later-born progenitor cell populations.

\section{HR- and NHEJ-Deficient Tumor Gene Expression Patterns}

Inactivation of $p 53$ in DSBR-deficient mice results in a drastic decrease in overall tumor-free survival, by the development of high-grade gliomas (Figure 3-3). Loss of p53 function was necessary for gliomagenesis in these mice, much like in many pediatric cases $[81,82,154,183]$. The data presented in my dissertation show the first analysis of a mouse model with early endogenous disruption of DSBR restricted to the cortex generating HGGs that resemble the pediatric disease.

Transcriptome analysis from RNA-sequencing data we collected on representative mouse HGG samples identified distinctive gene expression profiles associated with loss of HR or NHEJ. Predictably, many compensatory DNA repair genes were found to be significantly upregulated in (Brca2;p53) Emxl-cre gliomas, including Tdp1, Prkdc, Alkbhd2, Brcc3 and Atrx (Figure 3-4), while (Lig4;p53)Emxl-cre tumors had a significant upregulation of kinases, like $C C N D 3$ and $M A P 3 K 1$, which are specific to cancer development and tumor progression [103, 134, 193]. The differential gene expression patterns between Brca2- and Lig4-deficient gliomas highlight the importance of HR for DNA repair fidelity in replicating tumor cells. Without proper HR, cells must rely on other DNA damage repair pathways, like NHEJ and SSBR, for repair in order to proliferate and survive. In the case of Lig4-inhibition the cells have intact HR in early replicating cells, therefore errors in DNA repair resulting in mutations favoring tumorigenesis probably occur later in embryonic development. Genomic analysis of RNA-seq data identified recurrent chromosomal rearrangements, or potential genefusions, and amplifications in DSBR-deficient mouse gliomas (Figure 3-5) that are reflective of human brain tumors [90, 199]. The most commonly found gene-fusions involved Pdgfro, Braf and Met, and were more prevalent in (Brca2;p53) Emx1-cre gliomas. Whole exome sequencing to identify the average number of somatic mutations revealed that (Brca2;p53) Emx1-cre tumors had almost three times as many mutations than (Lig4;p53) Emxl-cre tumors. This data suggests that loss of HR results in greater accumulation of mutations, some of which were specific gene-fusion alterations that are known to cause HGGs. This is in all likelihood due to the higher frequency of errors in DNA repair through NHEJ culminating in more mutations. Earlier born progenitors 
especially require effective DSBR because any errors or resulting mutations have the potential to pass onto progeny cells increasing the possibility for devastating consequences, i.e. tumorigenesis.

The difference at E15.5 in cortical DNA damage is primarily due to the expansion of progenitor cells undergoing differentiation and maturation into post-mitotic neurons. As previously stated, NHEJ is the main form of DSBR once progenitors exit the cell cycle, because HR is only functional in replicating cells. Loss of NHEJ in the maturing neurons is detrimental because these cells must rely on a less efficient form of DSBR, altNHEJ, and other types of repair like SSBR, BER and NER $[45,46]$. Our lab has previously shown that Lig $4^{\text {Nes-cre }}$ mice accumulate $\gamma \mathrm{H} 2 \mathrm{AX}$ throughout the brain with age [12], indicating the other repair pathways are insufficient for repair. Therefore, in this model we see that Lig4-deficiency and NHEJ impairment results in a large accumulation of DNA damage in the upper cortical layers consisting of more mature, non-cycling cells (Figure 3-6). Although we expected more damage would result in more mutations leading to increased tumorigenesis, the difference in total amount and location of DNA damage surprisingly did not affect tumor latency or overall survival between either DSBR-deficient mouse models. Our findings underline the general importance of both DSBR pathways in providing genome stability to specific cortical cell populations during early development to prevent gliomagenesis.

\section{H3K27M Increases Genome Instability and Accelerates Gliomagenesis}

Histone $\mathrm{H} 3$ mutations are critical events in pediatric high-grade gliomagenesis $[80-82,90,248]$. The most common histone mutations found in these gliomas are $\mathrm{H} 3 \mathrm{~K} 27 \mathrm{M}$ and $\mathrm{H} 3 \mathrm{G} 34 \mathrm{R} / \mathrm{V}$ with both affecting distinct brain regions and developing at particular time periods. The discovery that DSBR-deficient mice with coincident $p 53$ inactivation develop HGGs, lead us to investigate the effect of H3K27M mutation in DSBR-deficient background. We hypothesized that the histone mutation may affect chromatin conformation leading to increased DNA damage susceptibility as part of the mechanism leading to glioma formation. My studies provide evidence for a novel role of $\mathrm{H} 3 \mathrm{~K} 27 \mathrm{M}$ in gliomagenesis, providing a second perspective on the mechanism by which loss of $\mathrm{H} 3 \mathrm{~K} 27 \mathrm{me} 3$, via $\mathrm{H} 3 \mathrm{~K} 27 \mathrm{M}$ mutation, may contribute to cancer development. In testing this, we found that expression of the H3K27M mutation in the neocortex causes cortical progenitors to impede efficient DSBR and alter genome homeostasis. This was shown by genome instability in H3K27M progenitor cells and association with DSBR (HR/NHEJ) deficiency and increased tumorigenesis (Figures 4-2 and 4-3). The observed increased frequency of DNA damage with or without additional DSBR mutations suggest that the genome of $\mathrm{H} 3 \mathrm{~K} 27 \mathrm{M}$ progenitor cells is more susceptible to damage and poised for gene alterations, with the consistent loss of $\mathrm{H} 3 \mathrm{~K} 27 \mathrm{me} 3$, from the earliest periods of development through gliomagenesis (Figure 4-1).

Early cortical development is associated with replication stress which has shown to result in preferential sensitivity to common fragile sites [249, 250]. Our results are consistent with previous findings that indicate H3K27M mutations are not sufficient on 
their own to induce tumorigenesis $[156,160]$ but rather put cortical progenitor cells in a poised pre-cancerous state characterized by bivalent gene promoter expression, replication stress and spontaneous DNA damage, where deficient DSBR promotes genome instability (Figure 4-9). Progenitor cells harboring gene aberrations are likely to undergo cell death unless tumor suppressors (i.e. p53) are inactivated or oncogenes are activated [147, 156, 160, 164]; however, widespread loss of H3K27me3 and/or H3K27M-induced replication stress could largely enhance accessibility to malignancy of the pre-cancerous progenitors and help them to avoid apoptosis, as shown in (p53;H3K27M) Emxl-cre mice having substantially high levels of DNA damage (Figure 4-3). p53 inactivation in DSBR-deficient mice display significantly decreased apoptosis; similarly, $H 3 K 27 M^{\text {Emx } 1-c r e}$ mice alone accrue DNA damage but do not develop HGGs and require $p 53$ inhibition to drive tumorigenesis. Although, these results were not that unexpected, as $\mathrm{H} 3 \mathrm{~K} 27 \mathrm{M}$ mutations are found in over $70 \%$ of DIPG patients [81, 82], exogenous expression of $\mathrm{H} 3 \mathrm{~K} 27 \mathrm{M}$ alone has been shown to be insufficient to induce neoplastic transformation of neural progenitor cells $[155,156]$. The combination of additional key mutations in oncogenes (PDGFR $\alpha$ ) and/or tumor suppressors (p53) are almost always required to promote tumorigenesis [81, 82, 155, 156, 160]. Our model uses an endogenous targeted gene expression method to activate the H3K27M mutation and inactivate DSBR genes (Brca2/Lig4) and p53 at one of the earliest known viable time points in the mouse brain. One limitation of this model is its specificity to the cortex and not the pons/midbrain where H3K27M related DIPGs are traditionally identified [160], nevertheless this model still produces HGGs that resemble the pediatric disease. This study, along with others, support a consistent role that $\mathrm{H} 3 \mathrm{~K} 27 \mathrm{M}$ induces tumor formation $[155-157,160,162]$.

One key question that still remains unanswered is the effect of the related histone $\mathrm{H} 3$ mutation, H3G34R/V. Since H3G34R/V mutations are typically found in the cortex of older pHGG patients and some aHGGs, it would seem that a mouse model driving gene expression only to the cortex would be the perfect candidate to study this specific oncohistone. In an identical manner to the H3K27M model, the H3G34R conditional knock-in mutation was created separately by Suzanne Baker's group. Interestingly, in crossing H3G34R ${ }^{\text {Emxl-cre }}$ with p53-, Brca2-, Lig4-conditional gene ablation we were unable to generate consistent HGGs that had latency differences to the (Brca2;p53)Emx1-cre

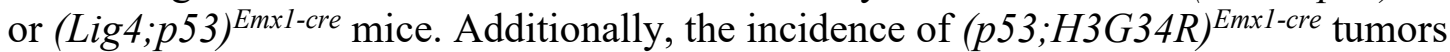

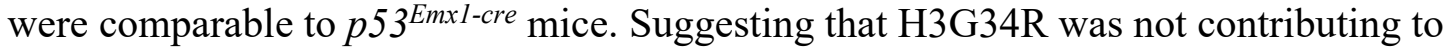
tumorigenesis in any substantial manner. The inability of H3G34R to influence tumorigenesis in physiological settings, like mouse models, is consistent with what other groups have observed [154]. The explanation behind this is speculative and could be due to multiple reasons, including the specific targeted cell population and/or the timing of mutational gene activation. Although this question remains unanswered, it leaves much opportunity for further investigations.

We identified global reduction of $\mathrm{H} 3 \mathrm{~K} 27 \mathrm{me} 3$ in cortical embryonic tissue at E12.5 and E15.5 in the H3K27M mutant mice (Figure 4-1), which is a well reported epigenetic modification attributed to this mutation [147, 163, 205]. To our knowledge, this is the earliest evidence showing the immediate effects of endogenous expression of 
this histone mutation in a mouse model. Additionally, we found a modest increase of K27 acetylation within specific upper and deep neocortical layers of the H3K27M mice, suggesting an increase in gene activation. Histological analysis revealed a similar loss of H3K27me3 expression in all H3K27M mutant tumors, in combination with or without Brca2-, Lig4- and p53-inactivation. These results suggest the effects of H3K27M on the epigenome are present at the earliest stages of development and carry all the way through development into gliomagenesis. Therefore, activation of the H3K27M mutation via Emxl-cre recapitulates the consequence of this oncohistone as seen in other scenarios, independent of DSBR-deficiency.

\section{H3K27M and DSBR-Deficiency Increase DNA Damage and Tumorigenesis}

The impact of the H3K27M mutation towards tumorigenesis in either (Brca2;p53) ${ }^{\text {Emxl-cre }}$ or (Lig4;p53) Emx1-cre was assessed by the generation of

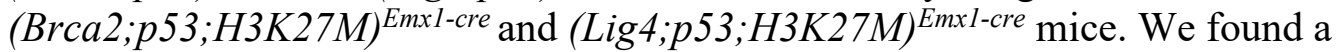
pronounced acceleration of tumor occurrence with a decrease in survival by about 4 months in the mice with the H3K27M mutation (Figure 4-2). Interestingly, (Brca2;p53;H3K27M) ${ }^{\text {Emx } 1-c r e ~ m i c e ~ s u c c u m b e d ~ t o ~ H G G s ~ o n ~ a v e r a g e ~ a b o u t ~ t w o ~ w e e k s ~}$ earlier than (Lig4;p53;H3K27M) Emxl-cre mutants, suggesting a differential link between

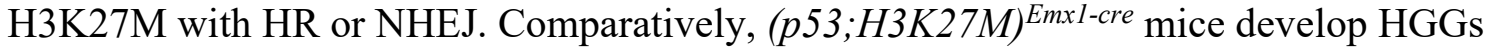
more quickly than the DSBR-deficient mutants alone but not as rapidly or as frequent as the triple mutants. Furthermore, the presence of the H3K27M mutation increased tumor diffusion, supporting its known role in driving diffuse high-grade gliomagenesis [81, 82, $155,160]$. Phenotypically, mice carrying H3K27M were significantly smaller in overall size compared to littermate controls until at least weaning age at P21, indicating a defect in early growth development. In addition to initial delay in growth, these mice demonstrated extreme hyperactive behavior throughout their lifespan. One possible explanation for hyperactivity could be due to the loss of the repressive mark, H3K27me 3 and consequently resulting in an increase of overall gene expression via activating marks like H3K27acylation and H3K4me3, causing their brains to almost go "haywire." Performing in vivo electrophysiology experiments could uncover a deeper understanding of the neuronal firing mechanisms that may also be causing this hyperactive behavior. One recent study linked commonly mutated PI3 kinase variants in glioblastomas to initiating brain hyperactivity during gliomagenesis [251].

Embryonic analysis identified significantly increased levels of DNA damage in mice harboring the H3K27M mutation (Figure 4-3), with selective differences in damage levels at E12.5 and E15.5 between the DSBR-deficient mutants, similar to what was found in mice without the histone mutation. At both embryonic timepoints analyzed

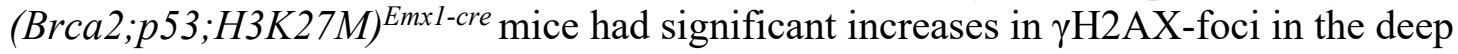
cortical layers compared to all other genotypes. Comparatively, $($ Lig4;p53;H3K27M) EmxI${ }^{c r e}$ showed no amplification in damage with H3K27M in the lower highly proliferative cortical cell populations, reiterating the importance of functional HR repair in early-born cortical progenitors. Remarkably, at E12.5 the levels of damage in the $(p 53 ; H 3 K 27 M)^{E m x l-c r e}$ mouse alone were significantly higher than an NHEJ-deficient 
mouse, (Lig4;p53)Emxl-cre , and as equally high as both (Lig4;p53;H3K27M)Emx1-cre and (Brca2;p53) Emx1-cre mice. Furthermore, at E15.5 we found the addition of the H3K27M mutation with Lig4-deficiency intensified DNA damage only in the upper differentiated (post-mitotic) cortical cell layers, which was nearly double the amount as the deep layers. Interestingly, when combining the total amount of DNA damage, both deep and upper layers combined, all mice with H3K27M had more $\gamma \mathrm{H} 2 \mathrm{AX}$-foci than their H3WT counterparts at E15.5. Taken together this implies that the H3K27M mutation is acting in a non-specific manner intensifying genome instability, which contributes to accelerating tumorigenesis, particularly in the presence of DSBR-deficiency.

\section{The Role of H3K27M in Genome Instability}

One of the most interesting findings from the embryonic DNA damage analysis

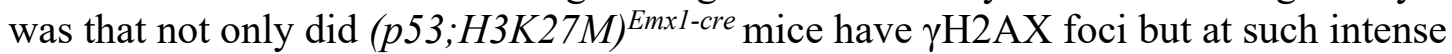
levels. At E12.5 the levels of damage were significantly higher than an NHEJ-deficient mouse, (Lig4;p53) Emxl-cre , and as equally high as both (Lig4;p53;H3K27M) ${ }^{\text {Emxl-cre }}$ and (Brca2;p53) Emxl-cre mice. However, at E15.5, (p53;H3K27M) Emx1-cre mice only had more damage than their counterparts, $p 53^{\text {Emxl-cre }}$, which was mostly confined to the deep proliferative cells. This discovery was somewhat of a surprise because it suggested for the first time, in a physiological setting, that H3K27M is associated with genome instability. We can only speculate on how mechanistically this could be possible, but one explanation is that the histone mutation may impair core subunits of the PRC2 complex, like EZH2, resulting in a stable chromatin conformational change [151]. Recent studies show this could be through inhibition of EZH2 automethylation, a process where EZH2 increases its histone methyltransferase activity to attain high enough levels of $\mathrm{H} 3 \mathrm{~K} 27 \mathrm{me} 3$, thereby creating a conformational change that is normally required for EZH2 activity [252-254]. This change conceivably results in a more open chromatin structure which has increased susceptibility to DNA damage [207-209]. Because histone mutations occur on the N-terminal tails, that are unstructured regions undergoing extensive posttranslational modifications, these alterations consequently recruit effector proteins that directly control transcriptionally active (open) or silent (closed) chromatin states [248]. Additional evidence through ATAC-seq in other H3K27M models have shown more open chromatin states directly due to the histone mutation [150], which in theory allows for more susceptibility to DNA damage. A simplistic interpretation of this is that a more open chromatin conformation state prolongs DNA exposure to damage inducing replicative stress, while a more closed, heterochromatin state, offers more protection from these stresses $[255,256]$. Interestingly, euchromatin is more sensitive to DNA damage caused by chemical agents, like cisplatin [255]. This opens the possibilities of exploring the use of different DNA damage inducing agents to treat pHGG patients, especially with histone mutations. Although, this is an extremely complex subject with many obstacles to overcome, including the blood brain barrier, it is a focus being studied by others $[55,94$, 257].

Further evidence suggests, that acetylation and/or a decrease in methylation levels promote more open or "relaxed" chromatin structure [209, 258, 259], which aligns with 
the epigenetic modifications that $\mathrm{H} 3 \mathrm{~K} 27 \mathrm{M}$ produces. One recent study found that in human dermal fibroblasts H3K27 methylation facilitates NHEJ through FANCD2 [260]. This study showed that transduced H3.1 K27M mutant cells had robust 53BP1 foci (DNA damage marker), increased genomic alterations and increased p53-dependent apoptosis. Likewise, Janet Partridge's lab showed in a fission yeast model, cells expressing the histone H3G34R mutation caused a defect in response to S-phase induced DNA damage resulting in defective HR and genome instability [261]. Evidence like this, suggests that H3K27M could be acting in a more direct manner towards DDR by inhibiting HR. Our data supports that HR is the more probable targeted repair pathway as the levels of

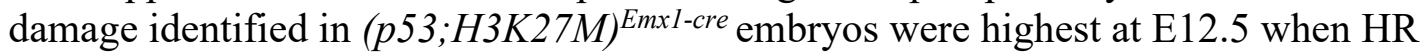
fidelity is most important. In addition, at E15.5 the vast majority of damage resulting from these mice were in the deeper proliferative cortical layers similar to the Brca2deficient embryos. Although this does not offer a complete explanation into the mechanisms of H3K27M associated DNA damage, it does provide a probable novel role that H3K27M plays in tumorigenesis. Additionally, this presents some insight for additional research studies to further explore the relationship not only between histone mutations and DNA damage repair but also gliomagenesis and possible therapeutic targets.

The increased levels of DNA damage from the H3K27M mutation that we observed, stemmed the hypothesis that there would be an increase of checkpoint activation in apical cortical progenitors, similar to findings from mice without H3K27M. At E12.5 we found that H3K27M associated DNA damage induced G2/M checkpoint in both (Brca2;p53;H3K27M) Emxl-cre and (p53;H3K27M) Emxl-cre (Figure 4-4). (Lig4;p53;H3K27M) Emx1-cre and (Lig4;p53) Emxl-cre mice had similar amounts of mitotic positive cells so this effect could be directly associated to NHEJ-deficiency. Additionally, at E15.5 we found similar levels of G2/M checkpoint induction in all genotypes in the apical cortical progenitor cells, with a slight increase in (Brca2; p53;H3K27M) Emxl-cre $^{\text {E }}$ mice. Due to the large cortical expansion at E15.5 we were able to additionally identify a small population of basal progenitors undergoing mitosis using $\mathrm{pH} 3$ and $\mathrm{Tbr} 2$ markers. Studies have shown that directly reducing H3K27me3 on the Eomes locus, a key basal progenitor regulator of Tbr2 in NSCs of mice, not only reduces the number of Tbr2 positive cells in the cortex but also directly decreases the amount of basal progenitors undergoing mitosis [106, 210, 211]. Granted the number of mitotic positive cells in this particular region are far and few between, we still found a reduction in all cohorts containing the H3K27M mutation. This provides evidence that the increase in H3K27Massociated DNA damage induces the G2/M cell cycle checkpoint in attempt to repair broken DNA strands, specifically in early-born apical cortical progenitor cells.

\section{H3K27M Drives Unique Gene Expressions Between HR- and NHEJ-Deficiency}

Gene expression analysis, via RNA-seq, identified expression changes that were not only unique to H3K27M tumors but further differentiated the H3K27M-DSBRdeficient mouse tumors from each other. Unsupervised hierarchical clustering and

principle component analysis revealed that all H3K27M tumors group together more than 
H3WT tumors, suggesting that $\mathrm{H} 3 \mathrm{~K} 27 \mathrm{M}$ may be affecting similar genes which are actually reducing tumor heterogeneity (Figure 4-5). Although H3K27M pHGGs have traditionally been defined as highly heterogeneous [125, 184, 262], recent studies analyzing large amounts of pediatric glioma samples have identified an assortment of additional commonly mutated genes within these tumors [80, 90, 120, 141]. Accordingly, we identified many gene alterations in $\mathrm{H} 3 \mathrm{~K} 27 \mathrm{M}$ mouse gliomas that were similar to the mutations found in pHGGs, such as: Egfr, Fgfr2, Lin28b and Pten (Figure 4-8). Lin28b and Plag1 are both oncogenes genes important for regulating NPC proliferation and differentiation [263-266] and we, along with others, identified both as upregulated in H3K27M mouse gliomas [156, 160]. Interestingly, (Lig4;p53;H3K27M) ${ }^{\text {Emxl-cre tumors }}$ had downregulation of the tumor suppressor $C d k n 2 a$, which has been associated with residual PRC2 activity and dysregulation in many H3K27M mouse models and DIPG cell lines $[90,155,157,160,205,267]$.

One explanation offered for dysregulated gene expression patterns in $\mathrm{H} 3 \mathrm{~K} 27 \mathrm{M}$ tumors is that the loss of $\mathrm{H} 3 \mathrm{~K} 27 \mathrm{me} 3$-repression is overpowered by selective recruitment of residual $\mathrm{PRC} 1 / 2$ activity on gene loci resulting in downregulation [160]. This argument is strengthened when analyzing the most downregulated genes in the H3K27M groups were homeodomain transcription factors such as: homeobox genes Hoxa gene cluster (Hoxa2-7 and 10) were identified. Hoxa genes are usually tightly regulated by transcriptional repressors for proper embryonic development (neurogenesis and differentiation) [212] and these genes by contrast were upregulated in H3WT tumors (Figure 4-5). Kristian Helin's group also identified reduced expression of Hoxa genes (Hoxa3-6) in H3K27M NSCs. They speculated that based on the identity of Hoxa genes, H3K27M may lead to a selection of a specific cell type during the transformation process wherein these cells are silenced by polycomb-group (PcG) proteins [157]. Another recent study showed that reduced H3K27me3 altered Hox gene expression resulting in neural tube defects [268]. This suggests that H3K27M may preferentially downregulate Hoxa genes through the loss of $\mathrm{H} 3 \mathrm{~K} 27 \mathrm{me} 3$ to more effectively control transcription and decrease cell differentiation. Furthermore, PRC1 components, which we also identified as upregulated in $\mathrm{H} 3 \mathrm{~K} 27 \mathrm{M}$, have been linked to stable repression of Hoxa genes [216]. Altogether this data suggests that a key mechanism of H3K27M may be to strengthen a transcriptional pattern specific to the cell of origin during the early stages of oncogenic transformation $[156,157]$.

Comparison of gene expression to other $\mathrm{H} 3 \mathrm{~K} 27 \mathrm{M}$ mouse models and patient samples $[82,155,157,160]$ revealed additional genes potentially contributing to H3K27M tumorigenesis and specifically identifies a subset of genes that are directly upregulated in many H3K27M expressing samples. These genes include Vcam 1, Agt, Peg3/10, Pbx3, Slit2, Ndn, Bmi 1, Id4, Eyal/2/4, Cdkn1c and Plag1 (Figure 4-5). Many of the genes have been implicated in assisting in tumorigenesis through various mechanisms and therefore could potentially be utilized as future therapeutic targets for glioma treatment [132, 219-222, 227]. In particular, Bmil, is an optimal target for therapy in these glioma models because its role has been well characterized in regulating H3K27me3 on chromatin and it has been shown to be recruited to DNA strand breaks contributing to H2A-ubiquitination and DNA repair [227, 269]. Gene targets such as 
Bmil, could be confirmed through protein and/or IHC markers or more thoroughly through chromatin immunoprecipitation [226]. Classification in gliomas would support preliminary studies using therapeutics to be tested in primary in vitro cultures. Early initial work from our lab has proven success in establishing primary cultures from mouse gliomas, which can be used for initial drug treatment trials. This could further lead to studies conducting the application of novel individual or previously established combinatorial drug treatments postnatally. DNA damage inducing drugs or treatments such as cisplatin, bleomycin, camptothecin, PARP-inhibitors and/or irradiation are commonly used to treat different forms of cancer [50, 144, 192, 225]. Therefore, these treatments and others could be optimized to target gliomas with DNA repair defects and/or histone mutations [144]. Some of the challenges of designing future therapeutic studies will be determining the optimal dosage to cross the blood brain barrier and the proper timed regimen as it is still unknown of when gliomas in these models would be best suited for treatment. Because of the rapid expansion of novel drugs targeting highly specific proteins, it is not inconceivable to imagine one day treatments targeting both DNA damage repair pathways and histone mutations could be utilized in pediatric brain tumor patients.

\section{H3K27M Alters the Cell of Origin}

In alignment with the known roles PRC1 and PRC2 play in modulating $\mathrm{H} 3 \mathrm{~K} 27 \mathrm{M}$ and H3K27me3, we found many upregulated genes from each complex where enriched in H3K27M gliomas, such as PRC1 (Ringlb and Bmil) and PRC2 (Ezh2, Suz12, and Jarid2) (Figure 4-5). Because we identified a profound loss of H3K27me3 embryonically in the histone mutants alone, we propose the upregulation of the PRC1/2 genes are directly linked to $\mathrm{H} 3 \mathrm{~K} 27 \mathrm{M}$ and not a universal effect of increased tumor proliferation. In connection to their roles in facilitating H3K27me3, Bmil and Ezh2, have also been closely linked to DSBR through regulation of HR, G2/M cell cycle arrest and p21 expression, which is involved in p53-dependent DNA damage checkpoint [269, 270]. Filbin et al. showed that PRC2 dysfunction in H3K27M gliomas contained overexpression of Bmil which drives a more OPC-like tumor population [120]. OPCs have been suggested to be the cell of origin for some types of pHGGs [120, 167, 168, 271]. Our IHC and gene expression analysis indicated OPCs or oligodendrocytes as convincing candidates for initiating cell populations in $\mathrm{H} 3 \mathrm{~K} 27 \mathrm{M}$ tumors especially with Brca2-deficiency (Figure 4-2). The cell of origin for gliomas has been widely studied but still many questions remain surrounding their identification in various brain tumors [91, 97, 98]. A better understanding of the potential cell type that is susceptible to specific oncogenic mutations will allow us to better develop more effective targeted treatment strategies.

Using IHC and various methods of glioma gene expression comparisons we attempted to assign potential neural and glial cell populations as originating cells for the HGGs. We found that (Lig4;p53) Emxl-cre tumors displayed more oligodendrocytic signatures in direct comparison to $(\mathrm{Brca} 2 ; \mathrm{p53})^{\text {Emxl-cre }}$ by gene expression analysis and Olig2 immunostaining. However, the addition of H3K27M expression with Lig4- or 
Brca2-inhibition resulted in increased expression of both astrocytic and astroglial signatures, suggesting a possible shift in cellular origin due to the histone mutation. Histological analysis confirmed a significant increase in astrocyte populations in

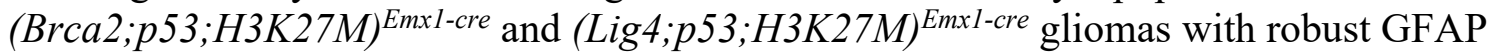
expression. (Brca2;p53;H3K27M) ${ }^{\text {Emx } 1-c r e}$ also displayed an increase in oligodendrocytes throughout the forebrain, indicating a highly mixed glial cell population. Embryonic analysis identified the loss of HR directly impacts earlier precursor cell populations in the lower cortical layers, these are among the most highly proliferative and earliest forming progenitor cells that have potential to give rise to neurons, astrocytes and OPCs, which can differentiate into oligodendrocytes. Conversely, NHEJ functions as the primary repair pathway in more differentiated and mature, lineage committed cell populations, such as astrocytes. Although preliminary, this data does provide insight into how the susceptibility of cortical progenitor cells, to oncogenic and tumor suppressor mutations, might contribute to gliomagenesis.

\section{H3K27M and NHEJ-Deficiency Effect Neural Development Genes}

All Lig4-deficient gliomas, with or without H3K27M, have an upregulation of genes involved in neural processes including neuron projection guidance, axon development, as well as cell fate commitment (Figure 4-7). These genes have slightly higher expression in the (Lig4;p53;H3K27M) ${ }^{\text {Emx I-cre }}$ group, which also have the greatest tumor diffusion among all analyzed mouse gliomas. The above neural processes are common among mature neurons in early cortical development where NHEJ (LIG4) is important for genome stability [21]. We found these processes were upregulated in glioma tissue samples, suggesting that dysregulation is carried through development and tumor cells are using these mechanisms in an advantageous manner. One interesting subset of genes within the upregulated neural processes were the Slit-Robo gene family members, including Slit1/2 and Robol/2. Post-translational modifications, like H3K4me, $\mathrm{H} 3 \mathrm{~K} 27 \mathrm{me}^{3}$ and H3K27 acetylation have been linked to regulation of Slit-Robo genes in terms cell migration and cancer invasion in the central nervous system [228]. Slit-Robo genes have also been identified as critical factors in neurogenesis and gliomagenesis [228, 272-274]. Additionally, a subgroup of protein tyrosine phosphatase (Ptp) genes Ptpru, Ptprn, and Ptpn5 were selectively upregulated in (Lig4;p53;H3K27M)EmxI-cre gliomas. PTPs are reported to be involved in regulation of a variety of cellular processes including cell growth, differentiation, mitotic cycle, oncogenic transformation, and glioma development $[129,235,236]$. Other groups have recently identified H3K27M DIPG patient tumors to contain upregulation of similar pathways involving neuron development, including axon guidance and genes associated with oligodendroglial differentiation [167]. Altogether this data suggests that Lig4-deficiency and even more so with $\mathrm{H} 3 \mathrm{~K} 27 \mathrm{M}$ expression, are selectively overexpressing genes that further promote tumor cell migration and diffusion. 


\section{H3K27M and Bivalent Gene Promoters}

The discovery of bivalent gene promoters [166] and recent evidence supporting H3K27M drives bivalent gene activation in similar mouse models $[160,162]$ sparked our interest to explore these signature patterns in the gliomas. We directly compared significantly up- and downregulated genes in the absence or presence of the H3K27M mutation from mouse glioma samples with a gene list generated by Suzanne Baker's group that defined histone marks on gene promoters from H3WT DIPGs [160]. Surprisingly, this revealed a similar percentage of upregulated bivalent genes in both H3K27M and H3WT gliomas, although H3K27M tumors had almost double the total number of bivalent genes (Figure 4-9). We were initially expecting to see a large increase in the proportion of upregulated genes in H3K27M gliomas compared to H3WT groups that were marked as bivalent promoters, which is what we found in (p53;H3K27M) $)^{\text {Emxl-cre }}$ gliomas compared to $p 53^{\text {Emxl-cre }}$ control tumors and this is reflective of what the Baker lab discovered. However, there was a striking difference in bivalent gene promoters when we compared $\mathrm{BrCa}$ - and Lig4-deficient tumors with or without H3K27M expression. The (Lig4;p53;H3K27M) Emxl-cre tumors had almost 4 times the amount of upregulated bivalent genes compared to (Brca2;p53;H3K27M) Emxl-cre (492 vs 136 genes, respectively). Remarkably, (Brca2;p53;H3K27M) ${ }^{\text {Emxl-cre }}$ had a larger percentage (46\%) of bivalent genes that were downregulated, which potentially stems from the original cortical cell populations that Brca2- and Lig4-deficiency most likely effect. When we compared H3WT tumors we found relatively no difference in percentage of bivalent gene promoters between DSBR-deficient gliomas, supporting the

role of H3K27M-mediated release of bivalent promoters. Even though (Brca2;p53) Emxl-cre gliomas had slightly more overall downregulated bivalent genes than (Lig4;p53) Emxl-cre, they both had about the same percentage of bivalent genes throughout. These data support that bivalency release via $\mathrm{H} 3 \mathrm{~K} 27 \mathrm{me} 3$ reduction at promoter regions is a highly probable mechanism for how $\mathrm{H} 3 \mathrm{~K} 27 \mathrm{M}$ facilitates differential gene expression signatures which are important for gliomagenesis.

Although the research on bivalent gene promoters and their interaction with $\mathrm{H} 3 \mathrm{~K} 27 \mathrm{M}$ is relatively new, other groups have attempted to study this in cultured settings, including NPCs and NSCs. One study found that overexpression of H3K27M in human NPCs resulted in bivalency remaining at promoters and $\mathrm{H} 3 \mathrm{~K} 27 \mathrm{me} 3$ only being reduced in the gene bodies [156]. While another group showed that overexpressing H3K27M and H3WT in mouse NSCs resulted in a large amount of differentially expressed genes that were not linked to H3K27me3 in H3WT NSCs, indicating an indirect effect of H3K27M [157]. Our data of bivalent gene promoter analysis aligned more with the results produced from Baker lab models, which was likely due to the use of the same H3K27M conditional mouse that regulates the histone mutation at relevant physiological levels from its endogenous promoter. Although, arguably our models target different mouse neural cell populations during gene activation through the use of distinct crerecombinases and result in locationally diverse gliomas. These differences further support the probable direct impact $\mathrm{H} 3 \mathrm{~K} 27 \mathrm{M}$ expression has on poised bivalent gene promoters in a physiological setting. 


\section{H3K27M Expression Effects Neural Lineage}

The importance of histone marks, H3K4me3 and H3K27me3, at specific stages of neural lineage progression are known to have direct regulatory effects on neural development [106, 166]. Studies have shown that with further neural lineage restriction the likelihood of developing brain tumors decreases based on the cell of origin $[97,98]$. Therefore, we examined the link between the potential cell of origin and the histone methylation marks present at critical genes that may be responsible glioma formation in our model. Wieland Huttner's group had previously classified a range of cortical cell populations, including NECs, apical and basal radial glial progenitors/neurons, and neurons, through early development (E9.5-E14.5) for cell type-specific histone methylation patterns, like bivalent gene promoters [106]. They found genes related to axon guidance and neural function that were bivalent in the NEC state were then turned on (H3K4me3+ and H3K27me3-) throughout lineage progression and into the neuronal state. Based on the DNA damage data for embryonic cortical cell layers with Brca2-and Lig4-deficiency (Figure 4-10) we chose three of these cell populations, NECs, apical radial glia committed to a neurogenic lineage (aRG-N), and neurons to compare tumor expression data. The DNA damage spatial difference is important when defining the unique cellular populations effected. NEC and aRG-Ns are found in the lower VZ where the majority of the Brca2-deficient damage was discovered. While the upper cortex in the $\mathrm{CP}$ and IZ contains more mature and differentiated neurons where the primary amount of Lig4-deficient damage was identified. Because (Brca2;p53;H3K27M) ${ }^{\text {Emxl-cre }}$ and (Lig4;p53;H3K27M) Emxl-cre displayed the most drastic phenotypes, both in terms of DNA damage and gliomagenesis, and had the greatest differences between bivalent gene promoter patterns we chose to focus the analysis on these two cohorts. We found that

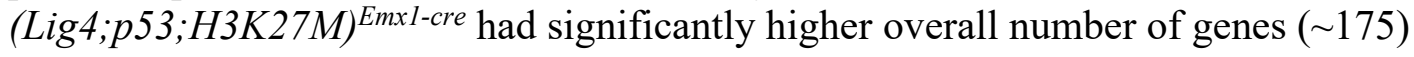
marked as bivalent at promoter regions through cortical development than (Brca2;p53;H3K27M) ${ }^{\text {Emxl-cre }}$ mice ( 5 genes). Moreover, (Lig4;p53;H3K27M) ${ }^{\text {Emx 1-cre }}$ had a high number of genes associated with aRG-Ns (140) and neurons (170) marked only with the gene activation associated mark, H3K4me3, which correlates with the overall increased gene expression we observed in these tumors. Both DSBR-deficient H3K27M mice displayed decreased bivalency through neural lineage progression which aligns with the current evidence suggesting genes that are bivalent in NECs are primed for expression and lose bivalency as they commit to cell states through differentiation and maturation.

One of the most interesting findings from this data set comparison was that (Brca2;p53;H3K27M) Emxl-cre mice had fewer total methylation marks in any category or cell type $(\sim 5)$ (Figure 4-10). This is probably a direct result of the DNA damage induced on the earlier cortical progenitor cell population that (Brca2;p53;H3K27M) ${ }^{\text {Emxl-cre mice }}$ display. The increased amount of genome instability in the apical progenitors reprograms and alters the pluripotency potential by decreasing bivalency within these cells that eventually transform into tumor cells. Furthermore, a recent study showed that DNA damage repair plays an essential role in cancer cell reprogramming that can go as far as reactivation of pluripotency genes [275], which suggest this process could be disrupted 
by DSBR-deficiency. Alternatively, Lig4-deficiency directly effects mature neurons that are already lineage committed and these cells contain genes with bivalent promoters that are more related to later neural development and tumorigenesis. Some of the most upregulated genes we identified were reflective of these pathways, including $P d g f \beta$, Sox 9 , and Syn2. Overexpression of $P d g f \beta$ in particular has been used in other mouse models to drive gliomagenesis [157]. Interestingly, through this analysis we were able to identify $C d k n 2 a$ as the only gene marked with $\mathrm{H} 3 \mathrm{~K} 4 \mathrm{me} 3$ throughout all neural lineage cell types as downregulated in the (Lig4;p53;H3K27M) $)^{\text {Emxl-cre }}$ tumors, indicating the probable early inactivation of this important tumor suppressor gene. The association of specific methylation marks on genes linked to neural development and tumorigenesis helps to provide some understanding of how mechanistically a combination of tumor related mutations, including Brca2, Lig4, p53 and H3K27M, may be cooperating in the earliest stages of corticogenesis to impose additional aberrations that ultimately cause gliomagenesis. Although this direct comparison of two different gene expression datasets from mouse tumors and mouse embryonic neural cells may not be the most ideal assessment, it nonetheless still gives insight into the potential mechanisms of gliomagenesis and opens up the possibility for future embryonic and post-natal cell sequencing experiments.

\section{Closing Remarks}

The research presented in my dissertation presents a new pHGG mouse model and examines the cooperation between genome instability and oncohistone mutations in gliomagenesis. Our data show that inhibition of DSBR by either Brca2-deficiency (HR) or Lig4-deficiency (NHEJ) in combination with $p 53$ inactivation results in gliomas resulting from elevated genome instability during embryogenesis. Furthermore, we found that $\mathrm{H} 3 \mathrm{~K} 27 \mathrm{M}$ expression promotes genome instability and accelerates cortical gliomagenesis, in the context of genome damage, from embryonic progenitors or neurons. We show that this is through upregulation of genes normally controlled by bivalent promoter post-translational modifications, like $\mathrm{H} 3 \mathrm{~K} 27 \mathrm{me} 3$ and $\mathrm{H} 3 \mathrm{~K} 4 \mathrm{me} 3$, which results in transcriptional alterations in genes important for both neural development and tumorigenesis. We suggest this occurs in specific populations of cortical cells that are more susceptible to DNA damage and transformations that may cause additional critical mutations during a limited timeframe of early cortical development which eventually result in pHGGs. Knowing that epigenetic regulation is tightly connected with neural development and differentiation, we propose that it is likely the specific genes affected by H3K27M expression may involve the developmental stage and cell state from which the glioma initiates. We believe this may contribute to the reduced heterogeneity in glioma expression signatures we found with $\mathrm{H} 3 \mathrm{~K} 27 \mathrm{M}$ in addition to either HR- or NHEJ-deficiency. Ultimately this work underscores the power of engineered mouse models as an approach to further understand the impact of genome instability leading to gliomagenesis. Furthermore, I hope these and other physiologically relevant high-grade gliomagenesis models will be useful for future mechanistic and preclinical therapy studies to develop effective treatments for these devastating diseases. A summary of these studies is presented as a working model in (Figure 5-1). 
Figure 5-1. Schematic of DSBR and H3K27M importance during early cortical development.

Stem and progenitor cells initiate corticogenesis around E10 in which they undergo a process of interkinetic nuclear migration where the nuclei of these cells (NEC) or radial glia (RG) cells occupy different positions along the apical-basal SVZ axis depending on the phase of the cell cycle. These are what we consider the "immature" cell population. In these cells DSBR is predominantly performed by HR (Brca2). H3K27M and the loss of bivalent promoters are also critical in these early progenitors. As cells progress through neural lineage they use RGs as scaffolding to migrate away from the apical surface and become differentiated/mature cortical neurons. Radial migration can be broken into four phases by unique cell types. Newborn neurons leave the proliferative areas (I) and reach the SVZ/IZ, where they attain a multipolar morphology (II). After pausing in the SVZ/IZ, cells migrate toward the $\mathrm{CP}$, using kinetic energy (III). At the end of their migration, cortical neurons switch to translocation of the soma (IV). During the transition to differentiation and maturation the cells exit the cell cycle and therefore rely on NHEJ (Lig4) for DSBR. The amount of DNA damage accumulation is primarily defined to the layers in which the cells are either HR- or NHEJ-deficient. When H3K27M is present it tends to effect more of the earlier cortical progenitors in the deep layers. The loss of H3K 27me3 (repressive) on the bivalent state drives increased gene expression with only $\mathrm{H} 3 \mathrm{~K} 4 \mathrm{me} 3$ (active) present. In addition, the active chromatin state is a more open structure which is more exposed to DNA damage resulting in increased genome instability. The combination of the H3K27M effects leads to accelerated gliomagenesis and decreased survival. All mutations induced with Emx1-cre at E9.5. E12-E18 is a robust period of cortical development. Red stars indicated timepoints of embryonic analysis (E12.5/E15.5). MZ, marginal zone; CP, cortical plate; IZ, intermediate zone; SVZ, subventricular zone; VZ, ventricular zone.

Modified with open access permission. Azzarelli, R., F. Guillemot, and E. Pacary, Function and regulation of Rnd proteins in cortical projection neuron migration. Front Neurosci, 2015. 9: p. 19.

Modified minimally from Cancer Cell, Vol:35/Issue:1, Larson, J.D., et al., Histone H3.3 K27M Accelerates Spontaneous Brainstem Glioma and Drives Restricted Changes in Bivalent Gene Expression., p. 140-155., Copyright (2019), with permission from Elsevier. 


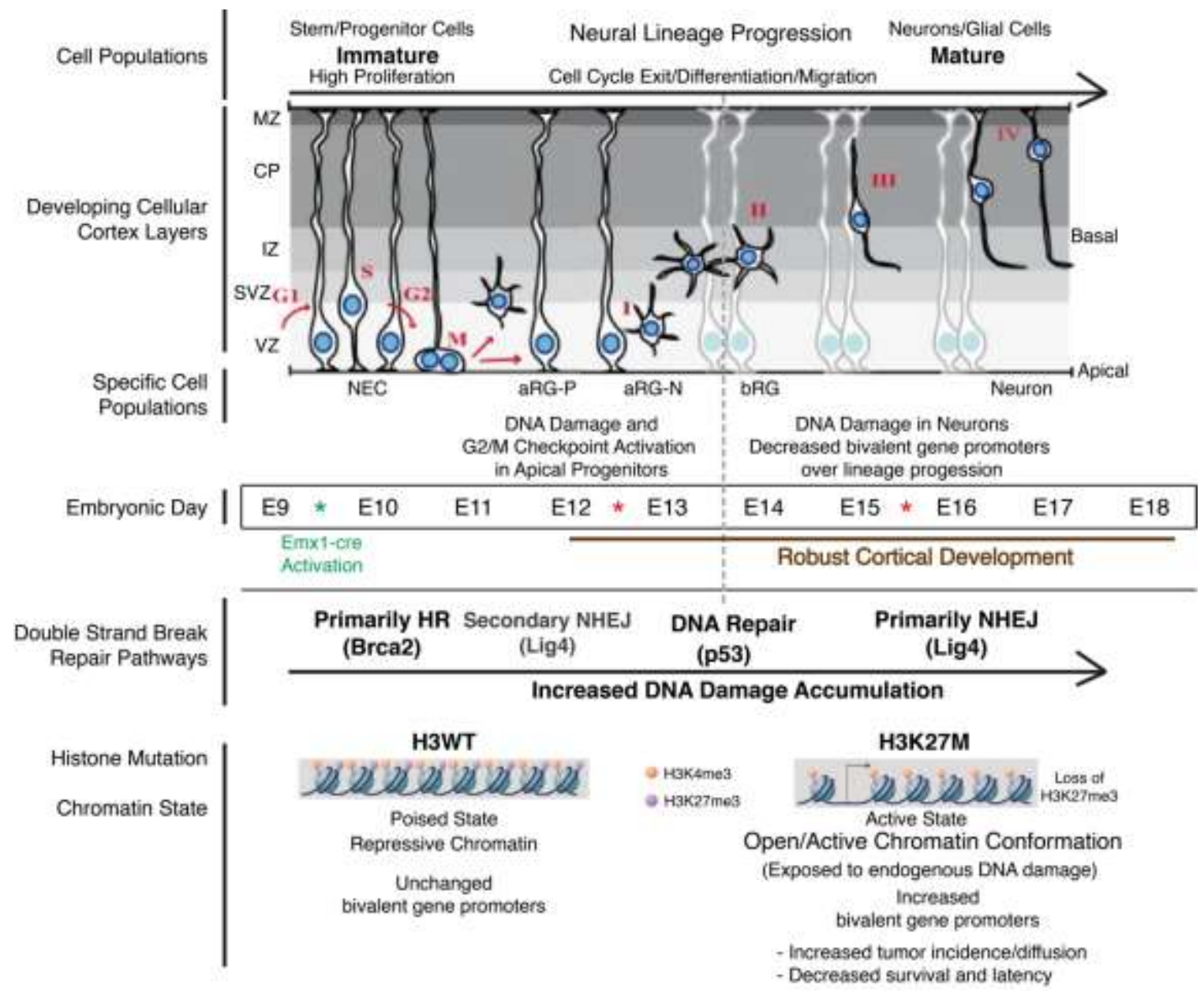




\section{LIST OF REFERENCES}

1. Hanahan, D. and R.A. Weinberg, Hallmarks of cancer: the next generation. Cell, 2011. 144(5): p. 646-74.

2. American Cancer Society. Cancer Facts \& Figures 2019. 2019; Available from: https://www.cancer.org/content/dam/cancer-org/research/cancer-facts-andstatistics/annual-cancer-facts-and-figures/2019/cancer-facts-and-figures-2019.pdf.

3. McKinnon, P.J., Maintaining genome stability in the nervous system. Nat Neurosci, 2013. 16(11): p. 1523-9.

4. McKinnon, P.J., Genome integrity and disease prevention in the nervous system. Genes Dev, 2017. 31(12): p. 1180-1194.

5. Madabhushi, R., L. Pan, and L.H. Tsai, DNA damage and its links to neurodegeneration. Neuron, 2014. 83(2): p. 266-282.

6. McKinnon, P.J., DNA repair deficiency and neurological disease. Nat Rev Neurosci, 2009. 10(2): p. 100-12.

7. Kass, E.M. and M. Jasin, Collaboration and competition between DNA doublestrand break repair pathways. FEBS Lett, 2010. 584(17): p. 3703-8.

8. Defelipe, J., The evolution of the brain, the human nature of cortical circuits, and intellectual creativity. Front Neuroanat, 2011. 5: p. 29.

9. Aguilera, A. and T. Garcia-Muse, $R$ loops: from transcription byproducts to threats to genome stability. Mol Cell, 2012. 46(2): p. 115-24.

10. Hanawalt, P.C. and G. Spivak, Transcription-coupled DNA repair: two decades of progress and surprises. Nat Rev Mol Cell Biol, 2008. 9(12): p. 958-70.

11. Enriquez-Rios, V., et al., DNA-PKcs, ATM, and ATR Interplay Maintains Genome Integrity during Neurogenesis. J Neurosci, 2017. 37(4): p. 893-905.

12. Shull, E.R., et al., Differential DNA damage signaling accounts for distinct neural apoptotic responses in ATLD and NBS. Genes Dev, 2009. 23(2): p. 171-80.

13. Nijhawan, D., N. Honarpour, and X. Wang, Apoptosis in neural development and disease. Annu Rev Neurosci, 2000. 23: p. 73-87.

14. Orii, K.E., et al., Selective utilization of nonhomologous end-joining and homologous recombination DNA repair pathways during nervous system development. Proc Natl Acad Sci U S A, 2006. 103(26): p. 10017-22.

15. Semple, B.D., et al., Brain development in rodents and humans: Identifying benchmarks of maturation and vulnerability to injury across species. Prog Neurobiol, 2013. 106-107: p. 1-16.

16. Hodge, R.D., et al., Conserved cell types with divergent features in human versus mouse cortex. Nature, 2019. 573(7772): p. 61-68.

17. Lodato, S. and P. Arlotta, Generating neuronal diversity in the mammalian cerebral cortex. Annu Rev Cell Dev Biol, 2015. 31: p. 699-720.

18. Spear, P.C. and C.A. Erickson, Interkinetic nuclear migration: a mysterious process in search of a function. Dev Growth Differ, 2012. 54(3): p. 306-16.

19. Molyneaux, B.J., et al., Neuronal subtype specification in the cerebral cortex. Nat Rev Neurosci, 2007. 8(6): p. 427-37.

20. Azzarelli, R., F. Guillemot, and E. Pacary, Function and regulation of Rnd proteins in cortical projection neuron migration. Front Neurosci, 2015. 9: p. 19. 
21. Caviness, V.S., Jr., R.S. Nowakowski, and P.G. Bhide, Neocortical neurogenesis: morphogenetic gradients and beyond. Trends Neurosci, 2009. 32(8): p. 443-50.

22. Chan, W.Y., et al., Proliferation and apoptosis in the developing human neocortex. Anat Rec, 2002. 267(4): p. 261-76.

23. Gotz, M. and W.B. Huttner, The cell biology of neurogenesis. Nat Rev Mol Cell Biol, 2005. 6(10): p. 777-88.

24. Arai, Y., et al., Neural stem and progenitor cells shorten S-phase on commitment to neuron production. Nat Commun, 2011. 2: p. 154.

25. Dehay, C. and H. Kennedy, Cell-cycle control and cortical development. Nat Rev Neurosci, 2007. 8(6): p. 438-50.

26. Hevner, R.F., Layer-specific markers as probes for neuron type identity in human neocortex and malformations of cortical development. J Neuropathol Exp Neurol, 2007. 66(2): p. 101-9.

27. Ferland, R.J., et al., Characterization of Foxp2 and Foxp1 mRNA and protein in the developing and mature brain. J Comp Neurol, 2003. 460(2): p. 266-79.

28. Hevner, R.F., et al., Tbrl regulates differentiation of the preplate and layer 6. Neuron, 2001. 29(2): p. 353-66.

29. Nieto, M., et al., Expression of Cux-1 and Cux-2 in the subventricular zone and upper layers II-IV of the cerebral cortex. J Comp Neurol, 2004. 479(2): p. 168-80.

30. West, S.C., Molecular views of recombination proteins and their control. Nat Rev Mol Cell Biol, 2003. 4(6): p. 435-45.

31. Krejci, L., et al., Homologous recombination and its regulation. Nucleic Acids Res, 2012. 40(13): p. 5795-818.

32. Wright, W.D., S.S. Shah, and W.D. Heyer, Homologous recombination and the repair of DNA double-strand breaks. J Biol Chem, 2018. 293(27): p. 1052410535.

33. Helleday, T., et al., DNA double-strand break repair: from mechanistic understanding to cancer treatment. DNA Repair (Amst), 2007. 6(7): p. 923-35.

34. Jasin, M. and R. Rothstein, Repair of strand breaks by homologous recombination. Cold Spring Harb Perspect Biol, 2013. 5(11): p. a012740.

35. Bakkenist, C.J. and M.B. Kastan, DNA damage activates ATM through intermolecular autophosphorylation and dimer dissociation. Nature, 2003. 421(6922): p. 499-506.

36. Herzog, K.H., et al., Requirement for Atm in ionizing radiation-induced cell death in the developing central nervous system. Science, 1998. 280(5366): p. 1089-91.

37. Cimprich, K.A. and D. Cortez, ATR: an essential regulator of genome integrity. Nat Rev Mol Cell Biol, 2008. 9(8): p. 616-27.

38. Wyman, C. and R. Kanaar, DNA double-strand break repair: all's well that ends well. Annu Rev Genet, 2006. 40: p. 363-83.

39. Moynahan, M.E. and M. Jasin, Mitotic homologous recombination maintains genomic stability and suppresses tumorigenesis. Nat Rev Mol Cell Biol, 2010. 11(3): p. 196-207.

40. Bassing, C.H. and F.W. Alt, The cellular response to general and programmed DNA double strand breaks. DNA Repair (Amst), 2004. 3(8-9): p. 781-96. 
41. Lieber, M.R., The mechanism of double-strand DNA break repair by the nonhomologous DNA end-joining pathway. Annu Rev Biochem, 2010. 79: p. 181211.

42. Davis, A.J., B.P. Chen, and D.J. Chen, DNA-PK: a dynamic enzyme in a versatile DSB repair pathway. DNA Repair (Amst), 2014. 17: p. 21-9.

43. Mahaney, B.L., K. Meek, and S.P. Lees-Miller, Repair of ionizing radiationinduced DNA double-strand breaks by non-homologous end-joining. Biochem J, 2009. 417(3): p. 639-50.

44. Williams, G.J., et al., Structural insights into NHEJ: building up an integrated picture of the dynamic DSB repair super complex, one component and interaction at a time. DNA Repair (Amst), 2014. 17: p. 110-20.

45. Bennardo, N., et al., Alternative-NHEJ is a mechanistically distinct pathway of mammalian chromosome break repair. PLoS Genet, 2008. 4(6): p. e1000110.

46. Chiruvella, K.K., Z. Liang, and T.E. Wilson, Repair of double-strand breaks by end joining. Cold Spring Harb Perspect Biol, 2013. 5(5): p. a012757.

47. Mao, Z., et al., DNA repair by nonhomologous end joining and homologous recombination during cell cycle in human cells. Cell Cycle, 2008. 7(18): p. 29026.

48. Iyama, T. and D.M. Wilson, 3rd, DNA repair mechanisms in dividing and nondividing cells. DNA Repair (Amst), 2013. 12(8): p. 620-36.

49. Frappart, P.O., et al., Recurrent genomic alterations characterize medulloblastoma arising from DNA double-strand break repair deficiency. Proc Natl Acad Sci U S A, 2009. 106(6): p. 1880-5.

50. Smith, J., et al., The ATM-Chk2 and ATR-Chk1 pathways in DNA damage signaling and cancer. Adv Cancer Res, 2010. 108: p. 73-112.

51. Rogakou, E.P., et al., DNA double-stranded breaks induce histone H2AX phosphorylation on serine 139. J Biol Chem, 1998. 273(10): p. 5858-68.

52. Scully, R. and A. Xie, Double strand break repair functions of histone H2AX. Mutat Res, 2013. 750(1-2): p. 5-14.

53. Stiff, T., et al., ATM and DNA-PK function redundantly to phosphorylate $H 2 A X$ after exposure to ionizing radiation. Cancer Res, 2004. 64(7): p. 2390-6.

54. Lobrich, M., et al., gammaH2AX foci analysis for monitoring DNA double-strand break repair: strengths, limitations and optimization. Cell Cycle, 2010. 9(4): p. 662-9.

55. Annovazzi, L., et al., The DNA damage/repair cascade in glioblastoma cell lines after chemotherapeutic agent treatment. Int J Oncol, 2015. 46(6): p. 2299-308.

56. Frappart, P.O., et al., An essential function for NBS1 in the prevention of ataxia and cerebellar defects. Nat Med, 2005. 11(5): p. 538-44.

57. Lee, Y. and P.J. McKinnon, Responding to DNA double strand breaks in the nervous system. Neuroscience, 2007. 145(4): p. 1365-74.

58. Frappart, P.O., et al., BRCA2 is required for neurogenesis and suppression of medulloblastoma. Embo Journal, 2007. 26(11): p. 2732-2742.

59. Katyal S., M.P.J., Mouse Models of DNA Double Strand Break Repair Deficiency and Cancer, in The DNA Damage Response: Implications on Cancer Formation and Treatment, S.Y. Khanna K., Editor. 2009, Springer, Dordecht. p. 285-305. 
60. Tronche, F., et al., Disruption of the glucocorticoid receptor gene in the nervous system results in reduced anxiety. Nat Genet, 1999. 23(1): p. 99-103.

61. Liang, H., S. Hippenmeyer, and H.T. Ghashghaei, A Nestin-cre transgenic mouse is insufficient for recombination in early embryonic neural progenitors. Biol Open, 2012. 1(12): p. 1200-3.

62. Lee, Y., et al., ATR maintains select progenitors during nervous system development. EMBO J, 2012. 31(5): p. 1177-89.

63. Brown, E.J. and D. Baltimore, ATR disruption leads to chromosomal fragmentation and early embryonic lethality. Genes Dev, 2000. 14(4): p. 397-402.

64. Kennedy, R.D. and A.D. D'Andrea, The Fanconi Anemia/BRCA pathway: new faces in the crowd. Genes Dev, 2005. 19(24): p. 2925-40.

65. Taniguchi, T. and A.D. D'Andrea, Molecular pathogenesis of Fanconi anemia: recent progress. Blood, 2006. 107(11): p. 4223-33.

66. Offit, K., et al., Shared genetic susceptibility to breast cancer, brain tumors, and Fanconi anemia. J Natl Cancer Inst, 2003. 95(20): p. 1548-51.

67. Shivji, M.K. and A.R. Venkitaraman, DNA recombination, chromosomal stability and carcinogenesis: insights into the role of BRCA2. DNA Repair (Amst), 2004. 3(8-9): p. 835-43.

68. Altmann, T. and A.R. Gennery, DNA ligase IV syndrome; a review. Orphanet J Rare Dis, 2016. 11(1): p. 137.

69. Ramaswamy, V., C. Nor, and M.D. Taylor, Erratum: p53 and Medulloblastoma. Cold Spring Harb Perspect Med, 2016. 6(4): p. a029579.

70. Lee, Y., et al., A molecular fingerprint for medulloblastoma. Cancer Res, 2003. 63(17): p. 5428-37.

71. Jonkers, J., et al., Synergistic tumor suppressor activity of BRCA2 and p53 in a conditional mouse model for breast cancer. Nature Genetics, 2001. 29(4): p. 418425.

72. Frappart, P.O. and P.J. McKinnon, Mouse models of DNA double-strand break repair and neurological disease. DNA Repair (Amst), 2008. 7(7): p. 1051-60.

73. Gorski, J.A., et al., Cortical excitatory neurons and glia, but not GABAergic neurons, are produced in the Emxl-expressing lineage. J Neurosci, 2002. 22(15): p. 6309-14.

74. Chou, S.J., et al., Lhx2 specifies regional fate in Emx1 lineage of telencephalic progenitors generating cerebral cortex. Nat Neurosci, 2009. 12(11): p. 1381-9.

75. Lee, Y., et al., Neurogenesis requires TopBP1 to prevent catastrophic replicative DNA damage in early progenitors. Nat Neurosci, 2012. 15(6): p. 819-26.

76. Pulvers, J.N. and W.B. Huttner, Brcal is required for embryonic development of the mouse cerebral cortex to normal size by preventing apoptosis of early neural progenitors. Development, 2009. 136(11): p. 1859-68.

77. Ostrom, Q.T., et al., CBTRUS Statistical Report: Primary Brain and Other Central Nervous System Tumors Diagnosed in the United States in 2012-2016. Neuro Oncol, 2019. 21(Suppl 5): p. v1-v100.

78. Merchant, T.E., I.F. Pollack, and J.S. Loeffler, Brain tumors across the age spectrum: biology, therapy, and late effects. Semin Radiat Oncol, 2010. 20(1): p. 58-66. 
79. Downing, J.R., et al., The Pediatric Cancer Genome Project. Nat Genet, 2012. 44(6): p. 619-22.

80. Mackay, A., et al., Integrated Molecular Meta-Analysis of 1,000 Pediatric HighGrade and Diffuse Intrinsic Pontine Glioma. Cancer Cell, 2017. 32(4): p. 520-537 e5.

81. Wu, G., et al., Somatic histone H3 alterations in pediatric diffuse intrinsic pontine gliomas and non-brainstem glioblastomas. Nat Genet, 2012. 44(3): p. 251-3.

82. Schwartzentruber, J., et al., Driver mutations in histone H3.3 and chromatin remodelling genes in paediatric glioblastoma. Nature, 2012. 482(7384): p. 22631.

83. Brennan, C.W., et al., The somatic genomic landscape of glioblastoma. Cell, 2013. 155(2): p. 462-77.

84. Cancer Genome Atlas Research, N., Comprehensive genomic characterization defines human glioblastoma genes and core pathways. Nature, 2008. 455(7216): p. 1061-8.

85. Parsons, D.W., et al., An integrated genomic analysis of human glioblastoma multiforme. Science, 2008. 321(5897): p. 1807-12.

86. Zhang, J., et al., Germline Mutations in Predisposition Genes in Pediatric Cancer. N Engl J Med, 2015. 373(24): p. 2336-2346.

87. Vogelstein, B., et al., Cancer genome landscapes. Science, 2013. 339(6127): p. 1546-58.

88. Jones, C. and S.J. Baker, Unique genetic and epigenetic mechanisms driving paediatric diffuse high-grade glioma. Nat Rev Cancer, 2014. 14(10).

89. Qaddoumi, I., I. Sultan, and A. Gajjar, Outcome and prognostic features in pediatric gliomas: a review of 6212 cases from the Surveillance, Epidemiology, and End Results database. Cancer, 2009. 115(24): p. 5761-70.

90. Wu, G., et al., The genomic landscape of diffuse intrinsic pontine glioma and pediatric non-brainstem high-grade glioma. Nat Genet, 2014. 46(5): p. 444-450.

91. Zong, H., L.F. Parada, and S.J. Baker, Cell of origin for malignant gliomas and its implication in therapeutic development. Cold Spring Harb Perspect Biol, 2015. 7(5).

92. Baker, S.J., D.W. Ellison, and D.H. Gutmann, Pediatric gliomas as neurodevelopmental disorders. Glia, 2016. 64(6): p. 879-95.

93. Ostrom, Q.T., et al., CBTRUS Statistical Report: Primary Brain and Other Central Nervous System Tumors Diagnosed in the United States in 2011-2015. Neuro Oncol, 2018. 20(suppl_4): p. iv1-iv86.

94. Michaeli, O. and U. Tabori, Pediatric High Grade Gliomas in the Context of Cancer Predisposition Syndromes. J Korean Neurosurg Soc, 2018. 61(3): p. 319332.

95. Cohen, K.J., et al., Temozolomide in the treatment of high-grade gliomas in children: a report from the Children's Oncology Group. Neuro Oncol, 2011. 13(3): p. 317-23.

96. Hoffman, L.M., et al., Clinical, Radiologic, Pathologic, and Molecular Characteristics of Long-Term Survivors of Diffuse Intrinsic Pontine Glioma (DIPG): A Collaborative Report From the International and European Society for Pediatric Oncology DIPG Registries. J Clin Oncol, 2018. 36(19): p. 1963-1972. 
97. Alcantara Llaguno, S.R. and L.F. Parada, Cell of origin of glioma: biological and clinical implications. Br J Cancer, 2016. 115(12): p. 1445-1450.

98. Alcantara Llaguno, S., et al., Cell-of-origin susceptibility to glioblastoma formation declines with neural lineage restriction. Nat Neurosci, 2019. 22(4): p. 545-555.

99. Visvader, J.E., Cells of origin in cancer. Nature, 2011. 469(7330): p. 314-22.

100. Alcantara Llaguno, S., et al., Malignant astrocytomas originate from neural stem/progenitor cells in a somatic tumor suppressor mouse model. Cancer Cell, 2009. 15(1): p. 45-56.

101. Chow, L.M., et al., Cooperativity within and among Pten, $p 53$, and Rb pathways induces high-grade astrocytoma in adult brain. Cancer Cell, 2011. 19(3): p. 30516.

102. Liu, C., et al., Mosaic analysis with double markers reveals tumor cell of origin in glioma. Cell, 2011. 146(2): p. 209-21.

103. Diaz, A.K. and S.J. Baker, The genetic signatures of pediatric high-grade glioma: no longer a one-act play. Semin Radiat Oncol, 2014. 24(4): p. 240-7.

104. Kessaris, N., et al., Competing waves of oligodendrocytes in the forebrain and postnatal elimination of an embryonic lineage. Nat Neurosci, 2006. 9(2): p. 1739.

105. Gallo, V. and B. Deneen, Glial development: the crossroads of regeneration and repair in the CNS. Neuron, 2014. 83(2): p. 283-308.

106. Albert, M., et al., Epigenome profiling and editing of neocortical progenitor cells during development. EMBO J, 2017. 36(17): p. 2642-2658.

107. Jacques, T.S., et al., Combinations of genetic mutations in the adult neural stem cell compartment determine brain tumour phenotypes. EMBO J, 2010. 29(1): p. 222-35.

108. Molofsky, A.V. and B. Deneen, Astrocyte development: A Guide for the Perplexed. Glia, 2015. 63(8): p. 1320-9.

109. Ge, W.P., et al., Local generation of glia is a major astrocyte source in postnatal cortex. Nature, 2012. 484(7394): p. 376-80.

110. Buczkowicz, P., et al., Genomic analysis of diffuse intrinsic pontine gliomas identifies three molecular subgroups and recurrent activating ACVR1 mutations. Nat Genet, 2014. 46(5): p. 451-6.

111. Paugh, B.S., et al., Novel oncogenic PDGFRA mutations in pediatric high-grade gliomas. Cancer Res, 2013. 73(20): p. 6219-29.

112. Radke, J., G. Bortolussi, and A. Pagenstecher, Akt and c-Myc induce stem-cell markers in mature primary $533(-) /(-)$ astrocytes and render these cells gliomagenic in the brain of immunocompetent mice. PLoS One, 2013. 8(2): p. e56691.

113. Endersby, R., et al., Nonredundant functions for Akt isoforms in astrocyte growth and gliomagenesis in an orthotopic transplantation model. Cancer Res, 2011. 71(12): p. 4106-16.

114. Bachoo, R.M., et al., Epidermal growth factor receptor and Ink4a/Arf: convergent mechanisms governing terminal differentiation and transformation along the neural stem cell to astrocyte axis. Cancer Cell, 2002. 1(3): p. 269-77. 
115. Ghazi, S.O., et al., Cell of origin determines tumor phenotype in an oncogenic Ras/p53 knockout transgenic model of high-grade glioma. J Neuropathol Exp Neurol, 2012. 71(8): p. 729-40.

116. Munoz, D.M., et al., Differential transformation capacity of neuro-glial progenitors during development. Proc Natl Acad Sci U S A, 2013. 110(35): p. 14378-83.

117. Fraser, M.M., et al., Pten loss causes hypertrophy and increased proliferation of astrocytes in vivo. Cancer Res, 2004. 64(21): p. 7773-9.

118. Dawson, M.R., et al., NG2-expressing glial progenitor cells: an abundant and widespread population of cycling cells in the adult rat CNS. Mol Cell Neurosci, 2003. 24(2): p. 476-88.

119. Geha, S., et al., NG2+/Olig2+ cells are the major cycle-related cell population of the adult human normal brain. Brain Pathol, 2010. 20(2): p. 399-411.

120. Filbin, M.G., et al., Developmental and oncogenic programs in H3K27M gliomas dissected by single-cell RNA-seq. Science, 2018. 360(6386): p. 331-335.

121. Verhaak, R.G., et al., Integrated genomic analysis identifies clinically relevant subtypes of glioblastoma characterized by abnormalities in PDGFRA, IDH1, EGFR, and NF1. Cancer Cell, 2010. 17(1): p. 98-110.

122. Rebetz, J., et al., Glial progenitor-like phenotype in low-grade glioma and enhanced CD133-expression and neuronal lineage differentiation potential in high-grade glioma. PLoS One, 2008. 3(4): p. e1936.

123. Lei, L., et al., Glioblastoma models reveal the connection between adult glial progenitors and the proneural phenotype. PLoS One, 2011. 6(5): p. e20041.

124. Barrett, L.E., et al., Self-renewal does not predict tumor growth potential in mouse models of high-grade glioma. Cancer Cell, 2012. 21(1): p. 11-24.

125. Paugh, B.S., et al., Integrated molecular genetic profiling of pediatric high-grade gliomas reveals key differences with the adult disease. J Clin Oncol, 2010. 28(18): p. 3061-8.

126. Pollack, I.F., et al., Rarity of PTEN deletions and EGFR amplification in malignant gliomas of childhood: results from the Children's Cancer Group 945 cohort. J Neurosurg, 2006. 105(5 Suppl): p. 418-24.

127. Li, G., et al., Expression of epidermal growth factor variant III (EGFRvIII) in pediatric diffuse intrinsic pontine gliomas. J Neurooncol, 2012. 108(3): p. 395402.

128. Zarghooni, M., et al., Whole-genome profiling of pediatric diffuse intrinsic pontine gliomas highlights platelet-derived growth factor receptor alpha and poly (ADP-ribose) polymerase as potential therapeutic targets. J Clin Oncol, 2010. 28(8): p. 1337-44.

129. Jones, D.T., et al., Recurrent somatic alterations of FGFR1 and NTRK2 in pilocytic astrocytoma. Nat Genet, 2013. 45(8): p. 927-32.

130. Jimenez-Pascual, A. and F.A. Siebzehnrubl, Fibroblast Growth Factor Receptor Functions in Glioblastoma. Cells, 2019. 8(7).

131. Ohashi, R., et al., Downregulation of fibroblast growth factor receptor 2 and its isoforms correlates with a high proliferation rate and poor prognosis in highgrade glioma. Oncol Rep, 2014. 32(3): p. 1163-9. 
132. Kraus, J.A., et al., Molecular genetic analysis of the TP53, PTEN, CDKN2A, EGFR, CDK4 and MDM2 tumour-associated genes in supratentorial primitive neuroectodermal tumours and glioblastomas of childhood. Neuropathol Appl Neurobiol, 2002. 28(4): p. 325-33.

133. Sherr, C.J. and F. McCormick, The RB and p53 pathways in cancer. Cancer Cell, 2002. 2(2): p. 103-12.

134. Paugh, B.S., et al., Genome-wide analyses identify recurrent amplifications of receptor tyrosine kinases and cell-cycle regulatory genes in diffuse intrinsic pontine glioma. J Clin Oncol, 2011. 29(30): p. 3999-4006.

135. Warren, K.E., Diffuse intrinsic pontine glioma: poised for progress. Front Oncol, 2012. 2: p. 205.

136. Wagner, S., et al., Treatment options in childhood pontine gliomas. J Neurooncol, 2006. 79(3): p. 281-7.

137. Louis, D.N., et al., The 2016 World Health Organization Classification of Tumors of the Central Nervous System: a summary. Acta Neuropathol, 2016. 131(6): p. 803-20.

138. Ceccarelli, M., et al., Molecular Profiling Reveals Biologically Discrete Subsets and Pathways of Progression in Diffuse Glioma. Cell, 2016. 164(3): p. 550-63.

139. Solomon, D.A., et al., Diffuse Midline Gliomas with Histone H3-K27M Mutation: A Series of 47 Cases Assessing the Spectrum of Morphologic Variation and Associated Genetic Alterations. Brain Pathol, 2016. 26(5): p. 569-80.

140. Lehnertz, B., et al., H3(K27M/I) mutations promote context-dependent transformation in acute myeloid leukemia with RUNX1 alterations. Blood, 2017. 130(20): p. 2204-2214.

141. Ryall, S., et al., Targeted detection of genetic alterations reveal the prognostic impact of H3K27M and MAPK pathway aberrations in paediatric thalamic glioma. Acta Neuropathol Commun, 2016. 4(1): p. 93.

142. $\mathrm{Wu}, \mathrm{L}$. , et al., The Bloom's syndrome gene product interacts with topoisomerase III. J Biol Chem, 2000. 275(13): p. 9636-44.

143. Yang, J., et al., Human topoisomerase IIIalpha is a single-stranded DNA decatenase that is stimulated by BLM and RMI1. J Biol Chem, 2010. 285(28): p. 21426-36.

144. Gojo, J., et al., Personalized Treatment of H3K27M-Mutant Pediatric Diffuse Gliomas Provides Improved Therapeutic Opportunities. Front Oncol, 2019. 9: p. 1436.

145. Goldberg, A.D., et al., Distinct factors control histone variant H3.3 localization at specific genomic regions. Cell, 2010. 140(5): p. 678-91.

146. Tagami, H., et al., Histone H3.1 and H3.3 complexes mediate nucleosome assembly pathways dependent or independent of DNA synthesis. Cell, 2004. 116(1): p. 51-61.

147. Bender, S., et al., Reduced H3K27me3 and DNA hypomethylation are major drivers of gene expression in K27M mutant pediatric high-grade gliomas. Cancer Cell, 2013. 24(5): p. 660-72.

148. Chan, K.M., et al., A lesson learned from the H3.3K27M mutation found in pediatric glioma: a new approach to the study of the function of histone modifications in vivo? Cell Cycle, 2013. 12(16): p. 2546-52. 
149. Lewis, P.W., et al., Inhibition of PRC2 activity by a gain-of-function H3 mutation found in pediatric glioblastoma. Science, 2013. 340(6134): p. 857-61.

150. Harutyunyan, A.S., et al., H3K27M induces defective chromatin spread of PRC2mediated repressive $H 3 \mathrm{~K} 27 \mathrm{me} 2 / \mathrm{me} 3$ and is essential for glioma tumorigenesis. Nat Commun, 2019. 10(1): p. 1262.

151. Stafford, J.M., et al., Multiple modes of PRC2 inhibition elicit global chromatin alterations in H3K27M pediatric glioma. Sci Adv, 2018. 4(10): p. eaau5935.

152. Margueron, R. and D. Reinberg, The Polycomb complex PRC2 and its mark in life. Nature, 2011. 469(7330): p. 343-9.

153. Kasper, L.H. and S.J. Baker, Invited Review: Emerging functions of histone H3 mutations in paediatric diffuse high-grade gliomas. Neuropathol Appl Neurobiol, 2020. 46(1): p. 73-85.

154. Pathania, M., et al., H3.3(K27M) Cooperates with Trp53 Loss and PDGFRA Gain in Mouse Embryonic Neural Progenitor Cells to Induce Invasive High-Grade Gliomas. Cancer Cell, 2017. 32(5): p. 684-700 e9.

155. Cordero, F.J., et al., Histone H3.3K27M Represses p16 to Accelerate Gliomagenesis in a Murine Model of DIPG. Mol Cancer Res, 2017. 15(9): p. 1243-1254.

156. Funato, K., et al., Use of human embryonic stem cells to model pediatric gliomas with H3.3K27M histone mutation. Science, 2014. 346(6216): p. 1529-33.

157. Mohammad, F., et al., EZH2 is a potential therapeutic target for H3K27M-mutant pediatric gliomas. Nat Med, 2017. 23(4): p. 483-492.

158. Subashi, E., et al., Tumor location, but not H3.3K27M, significantly influences the blood-brain-barrier permeability in a genetic mouse model of pediatric highgrade glioma. J Neurooncol, 2016. 126(2): p. 243-51.

159. Larson, J.D. and S.J. Baker, Engineering Inducible Knock-In Mice to Model Oncogenic Brain Tumor Mutations from Endogenous Loci. Methods Mol Biol, 2019. 1869: p. 207-230.

160. Larson, J.D., et al., Histone H3.3 K27M Accelerates Spontaneous Brainstem Glioma and Drives Restricted Changes in Bivalent Gene Expression. Cancer Cell, 2019. 35(1): p. 140-155 e7.

161. Bavle, A.A., F.Y. Lin, and D.W. Parsons, Applications of Genomic Sequencing in Pediatric CNS Tumors. Oncology (Williston Park), 2016. 30(5): p. 411-23.

162. Silveira, A.B., et al., H3.3 K27M depletion increases differentiation and extends latency of diffuse intrinsic pontine glioma growth in vivo. Acta Neuropathol, 2019. 137(4): p. 637-655.

163. Krug, B., et al., Pervasive H3K27 Acetylation Leads to ERV Expression and a Therapeutic Vulnerability in H3K27M Gliomas. Cancer Cell, 2019. 36(3): p. 338339.

164. Castel, D., et al., Histone H3F3A and HIST1H3B K27M mutations define two subgroups of diffuse intrinsic pontine gliomas with different prognosis and phenotypes. Acta Neuropathol, 2015. 130(6): p. 815-27.

165. Castel, D., et al., Transcriptomic and epigenetic profiling of 'diffuse midline gliomas, H3 K27M-mutant' discriminate two subgroups based on the type of histone H3 mutated and not supratentorial or infratentorial location. Acta Neuropathol Commun, 2018. 6(1): p. 117. 
166. Bernstein, B.E., et al., A bivalent chromatin structure marks key developmental genes in embryonic stem cells. Cell, 2006. 125(2): p. 315-26.

167. Nagaraja, S., et al., Histone Variant and Cell Context Determine H3K27M Reprogramming of the Enhancer Landscape and Oncogenic State. Mol Cell, 2019. 76(6): p. 965-980 e12.

168. Nagaraja, S., et al., Transcriptional Dependencies in Diffuse Intrinsic Pontine Glioma. Cancer Cell, 2017. 31(5): p. 635-652 e6.

169. Lee, Y. and P.J. McKinnon, DNA ligase IV suppresses medulloblastoma formation. Cancer Research, 2002. 62(22): p. 6395-6399.

170. Shimada, M., et al., Polynucleotide kinase-phosphatase enables neurogenesis via multiple DNA repair pathways to maintain genome stability. Embo Journal, 2015. 34(19): p. 2465-2480.

171. Dobin, A., et al., STAR: ultrafast universal RNA-seq aligner. Bioinformatics, 2013. 29(1): p. 15-21.

172. Anders, S., P.T. Pyl, and W. Huber, HTSeq--a Python framework to work with high-throughput sequencing data. Bioinformatics, 2015. 31(2): p. 166-9.

173. Subramanian, A., et al., Gene set enrichment analysis: a knowledge-based approach for interpreting genome-wide expression profiles. Proc Natl Acad Sci U S A, 2005. 102(43): p. 15545-50.

174. Hanzelmann, S., R. Castelo, and J. Guinney, GSVA: gene set variation analysis for microarray and RNA-seq data. BMC Bioinformatics, 2013. 14: p. 7.

175. Chen, E.Y., et al., Enrichr: interactive and collaborative HTML5 gene list enrichment analysis tool. BMC Bioinformatics, 2013. 14: p. 128.

176. Kuleshov, M.V., et al., Enrichr: a comprehensive gene set enrichment analysis web server 2016 update. Nucleic Acids Res, 2016. 44(W1): p. W90-7.

177. Tian, L., et al., CICERO: a versatile method for detecting complex and diverse driver fusions using cancer RNA sequencing data. Genome Biol, 2020. 21(1): p. 126.

178. Wei, L., et al., Exome sequencing analysis of murine medulloblastoma models identifies WDR11 as a potential tumor suppressor in Group 3 tumors. Oncotarget, 2017. 8(39): p. 64685-64697.

179. Ludwig, T., et al., Targeted mutations of breast cancer susceptibility gene homologs in mice: lethal phenotypes of Brca1, Brca2, Brca1/Brca2, Brca1/p53, and Brca2/p53 nullizygous embryos. Genes Dev, 1997. 11(10): p. 1226-41.

180. Sharan, S.K., et al., Embryonic lethality and radiation hypersensitivity mediated by Rad51 in mice lacking Brca2. Nature, 1997. 386(6627): p. 804-10.

181. Suzuki, A., et al., Brca2 is required for embryonic cellular proliferation in the mouse. Genes Dev, 1997. 11(10): p. 1242-52.

182. Fernandez-Capetillo, O., et al., H2AX: the histone guardian of the genome. DNA Repair (Amst), 2004. 3(8-9): p. 959-67.

183. Ozaki, T. and A. Nakagawara, Role of p53 in Cell Death and Human Cancers. Cancers (Basel), 2011. 3(1): p. 994-1013.

184. Huse, J.T., H.S. Phillips, and C.W. Brennan, Molecular subclassification of diffuse gliomas: seeing order in the chaos. Glia, 2011. 59(8): p. 1190-9. 
185. Bielen, A., et al., Enhanced efficacy of IGF1R inhibition in pediatric glioblastoma by combinatorial targeting of PDGFRalpha/beta. Mol Cancer Ther, 2011. 10(8): p. 1407-18.

186. Soroceanu, L., et al., Identification of IGF2 signaling through phosphoinositide-3kinase regulatory subunit 3 as a growth-promoting axis in glioblastoma. Proc Natl Acad Sci U S A, 2007. 104(9): p. 3466-71.

187. Bannykh, S.I., et al., Oligodendroglial-specific transcriptional factor SOX10 is ubiquitously expressed in human gliomas. J Neurooncol, 2006. 76(2): p. 115-27.

188. Ferletta, M., et al., Sox10 has a broad expression pattern in gliomas and enhances platelet-derived growth factor-B--induced gliomagenesis. Mol Cancer Res, 2007. 5(9): p. 891-7.

189. Wu, L., et al., BCL3 expression promotes resistance to alkylating chemotherapy in gliomas. Sci Transl Med, 2018. 10(448).

190. McKinnon, P.J. and K.W. Caldecott, DNA strand break repair and human genetic disease. Annu Rev Genomics Hum Genet, 2007. 8: p. 37-55.

191. Takashima, H., et al., Mutation of TDP1, encoding a topoisomerase I-dependent DNA damage repair enzyme, in spinocerebellar ataxia with axonal neuropathy. Nat Genet, 2002. 32(2): p. 267-72.

192. Pommier, Y., et al., Tyrosyl-DNA-phosphodiesterases (TDP1 and TDP2). DNA Repair (Amst), 2014. 19: p. 114-29.

193. Wang, H., et al., Deep multiomics profiling of brain tumors identifies signaling networks downstream of cancer driver genes. Nat Commun, 2019. 10(1): p. 3718.

194. Adamson, B., et al., A genome-wide homologous recombination screen identifies the RNA-binding protein RBMX as a component of the DNA-damage response. Nat Cell Biol, 2012. 14(3): p. 318-28.

195. Azzarelli, R., B.D. Simons, and A. Philpott, The developmental origin of brain tumours: a cellular and molecular framework. Development, 2018. 145(10).

196. Cahoy, J.D., et al., A transcriptome database for astrocytes, neurons, and oligodendrocytes: a new resource for understanding brain development and function. J Neurosci, 2008. 28(1): p. 264-78.

197. Gao, Y., et al., Interplay of 53 and DNA-repair protein XRCC4 in tumorigenesis, genomic stability and development. Nature, 2000. 404(6780): p. 897-900.

198. Ratnaparkhe, M., et al., Defective DNA damage repair leads to frequent catastrophic genomic events in murine and human tumors. Nat Commun, 2018. 9(1): p. 4760.

199. Xu, T., et al., Gene Fusion in Malignant Glioma: An Emerging Target for NextGeneration Personalized Treatment. Transl Oncol, 2018. 11(3): p. 609-618.

200. Knobbe, C.B., J. Reifenberger, and G. Reifenberger, Mutation analysis of the Ras pathway genes NRAS, HRAS, KRAS and BRAF in glioblastomas. Acta Neuropathol, 2004. 108(6): p. 467-70.

201. Loo, E., et al., BRAF V600E Mutation Across Multiple Tumor Types: Correlation Between DNA-based Sequencing and Mutation-specific Immunohistochemistry. Appl Immunohistochem Mol Morphol, 2018. 26(10): p. 709-713.

202. Ueda, R., et al., Identification of a human glioma antigen, SOX6, recognized by patients' sera. Oncogene, 2004. 23(7): p. 1420-7. 
203. Romagosa, C., et al., p16(Ink4a) overexpression in cancer: a tumor suppressor gene associated with senescence and high-grade tumors. Oncogene, 2011. 30(18): p. 2087-97.

204. Senturk, E. and J.J. Manfredi, p53 and cell cycle effects after DNA damage. Methods Mol Biol, 2013. 962: p. 49-61.

205. Chan, K.M., et al., The histone H3.3K27M mutation in pediatric glioma reprograms H3K27 methylation and gene expression. Genes Dev, 2013. 27(9): p. 985-90.

206. Krug, B., et al., Pervasive H3K27 Acetylation Leads to ERV Expression and a Therapeutic Vulnerability in H3K27M Gliomas. Cancer Cell, 2019. 35(5): p. 782797 e8.

207. Falk, M., E. Lukasova, and S. Kozubek, Chromatin structure influences the sensitivity of DNA to gamma-radiation. Biochim Biophys Acta, 2008. 1783(12): p. 2398-414.

208. Hauer, M.H. and S.M. Gasser, Chromatin and nucleosome dynamics in DNA damage and repair. Genes Dev, 2017. 31(22): p. 2204-2221.

209. Nair, N., M. Shoaib, and C.S. Sorensen, Chromatin Dynamics in Genome Stability: Roles in Suppressing Endogenous DNA Damage and Facilitating DNA Repair. Int J Mol Sci, 2017. 18(7).

210. Sessa, A., et al., Tbr2 directs conversion of radial glia into basal precursors and guides neuronal amplification by indirect neurogenesis in the developing neocortex. Neuron, 2008. 60(1): p. 56-69.

211. Arnold, S.J., et al., The T-box transcription factor Eomes/Tbr2 regulates neurogenesis in the cortical subventricular zone. Genes Dev, 2008. 22(18): p. 2479-84.

212. Ringrose, L. and R. Paro, Epigenetic regulation of cellular memory by the Polycomb and Trithorax group proteins. Annu Rev Genet, 2004. 38: p. 413-43.

213. Wan, Y.C.E., J. Liu, and K.M. Chan, Histone H3 Mutations in Cancer. Curr Pharmacol Rep, 2018. 4(4): p. 292-300.

214. van den Boom, V., et al., Non-canonical PRC1.1 Targets Active Genes Independent of H3K27me3 and Is Essential for Leukemogenesis. Cell Rep, 2016. 14(2): p. 332-46.

215. Lachmann, A., et al., ChEA: transcription factor regulation inferred from integrating genome-wide ChIP-X experiments. Bioinformatics, 2010. 26(19): $\mathrm{p}$. 2438-44.

216. Sakamoto, Y., et al., Overlapping roles of the methylated DNA-binding protein $M B D 1$ and polycomb group proteins in transcriptional repression of HOXA genes and heterochromatin foci formation. J Biol Chem, 2007. 282(22): p. 16391-400.

217. Mi, H., et al., PANTHER version 14: more genomes, a new PANTHER GO-slim and improvements in enrichment analysis tools. Nucleic Acids Res, 2019. 47(D1): p. D419-D426.

218. Barrett, T., et al., NCBI GEO: archive for functional genomics data sets--update. Nucleic Acids Res, 2013. 41(Database issue): p. D991-5.

219. Liu, Y.S., et al., MiR-181b modulates EGFR-dependent VCAM-1 expression and monocyte adhesion in glioblastoma. Oncogene, 2017. 36(35): p. 5006-5022. 
220. Perdomo-Pantoja, A., et al., Angiotensinogen rs5050 germline genetic variant as potential biomarker of poor prognosis in astrocytoma. PLoS One, 2018. 13(11): p. e0206590.

221. Ho, C.Y., et al., MicroRNA profiling in pediatric pilocytic astrocytoma reveals biologically relevant targets, including $P B X 3, N F I B$, and METAP2. Neuro Oncol, 2013. 15(1): p. 69-82.

222. Otsuka, S., et al., Aberrant promoter methylation and expression of the imprinted PEG3 gene in glioma. Proc Jpn Acad Ser B Phys Biol Sci, 2009. 85(4): p. 157-65.

223. Kainz, B., et al., Overexpression of the paternally expressed gene 10 (PEG10) from the imprinted locus on chromosome $7 q 21$ in high-risk B-cell chronic lymphocytic leukemia. Int J Cancer, 2007. 121(9): p. 1984-93.

224. Jeon, H.M., et al., Inhibitor of differentiation 4 drives brain tumor-initiating cell genesis through cyclin $E$ and notch signaling. Genes Dev, 2008. 22(15): p. 202833.

225. Hasegawa, K. and K. Yoshikawa, Necdin regulates p53 acetylation via Sirtuin1 to modulate DNA damage response in cortical neurons. J Neurosci, 2008. 28(35): p. 8772-84.

226. Abdouh, M., et al., BMI1 sustains human glioblastoma multiforme stem cell renewal. J Neurosci, 2009. 29(28): p. 8884-96.

227. Jin, X., et al., Targeting glioma stem cells through combined BMII and EZH2 inhibition. Nat Med, 2017. 23(11): p. 1352-1361.

228. Amodeo, V., et al., A PML/Slit Axis Controls Physiological Cell Migration and Cancer Invasion in the CNS. Cell Rep, 2017. 20(2): p. 411-426.

229. Auvergne, R.M., et al., Transcriptional differences between normal and gliomaderived glial progenitor cells identify a core set of dysregulated genes. Cell Rep, 2013. 3(6): p. 2127-41.

230. Ashburner, M., et al., Gene ontology: tool for the unification of biology. The Gene Ontology Consortium. Nat Genet, 2000. 25(1): p. 25-9.

231. Guerreiro Stucklin, A.S., et al., Alterations in ALK/ROS1/NTRK/MET drive a group of infantile hemispheric gliomas. Nat Commun, 2019. 10(1): p. 4343.

232. Cocco, E., M. Scaltriti, and A. Drilon, NTRK fusion-positive cancers and TRK inhibitor therapy. Nat Rev Clin Oncol, 2018. 15(12): p. 731-747.

233. Huang, R., et al., The NCATS BioPlanet - An Integrated Platform for Exploring the Universe of Cellular Signaling Pathways for Toxicology, Systems Biology, and Chemical Genomics. Front Pharmacol, 2019. 10: p. 445.

234. Kanehisa, M. and S. Goto, KEGG: kyoto encyclopedia of genes and genomes. Nucleic Acids Res, 2000. 28(1): p. 27-30.

235. Kharitonenkov, A., et al., A family of proteins that inhibit signalling through tyrosine kinase receptors. Nature, 1997. 386(6621): p. 181-6.

236. Navis, A.C., et al., Protein tyrosine phosphatases in glioma biology. Acta Neuropathol, 2010. 119(2): p. 157-75.

237. Ramdzan, Z.M. and A. Nepveu, CUX1, a haploinsufficient tumour suppressor gene overexpressed in advanced cancers. Nat Rev Cancer, 2014. 14(10): p. 67382.

238. Seidu, R.A., et al., Paradoxical Role of High Mobility Group Box 1 in Glioma: A Suppressor or a Promoter? Oncol Rev, 2017. 11(1): p. 325. 
239. Jadhav, U., et al., Replicational Dilution of H3K27me3 in Mammalian Cells and the Role of Poised Promoters. Mol Cell, 2020.

240. Rosenberg, A.B., et al., Single-cell profiling of the developing mouse brain and spinal cord with split-pool barcoding. Science, 2018. 360(6385): p. 176-182.

241. Mikkelsen, T.S., et al., Dissecting direct reprogramming through integrative genomic analysis. Nature, 2008. 454(7200): p. 49-55.

242. Meissner, A., et al., Genome-scale DNA methylation maps of pluripotent and differentiated cells. Nature, 2008. 454(7205): p. 766-70.

243. Gu, J., et al., Molecular epidemiology of primary brain tumors. Neurotherapeutics, 2009. 6(3): p. 427-35.

244. Wang, L.E., et al., Polymorphisms of DNA repair genes and risk of glioma. Cancer Res, 2004. 64(16): p. 5560-3.

245. Liu, Y., et al., Polymorphisms of LIG4 and XRCC4 involved in the NHEJ pathway interact to modify risk of glioma. Hum Mutat, 2008. 29(3): p. 381-9.

246. Laboratory, T.J. B6.129S2-Emx1tm1 (cre)Krj/J. 2020; Available from: https://www.jax.org/strain/005628.

247. Garcia-Arocena, D. Cre-lox myths busted. 2013; Available from: https://www.jax.org/news-and-insights/jax-blog/2013/september/cre-lox-mythsbusted.

248. Yuen, B.T. and P.S. Knoepfler, Histone H3.3 mutations: a variant path to cancer. Cancer Cell, 2013. 24(5): p. 567-74.

249. Glover, T.W., T.E. Wilson, and M.F. Arlt, Fragile sites in cancer: more than meets the eye. Nat Rev Cancer, 2017. 17(8): p. 489-501.

250. Irony-Tur Sinai, M. and B. Kerem, DNA replication stress drives fragile site instability. Mutat Res, 2018. 808: p. 56-61.

251. Yu, K., et al., PIK3CA variants selectively initiate brain hyperactivity during gliomagenesis. Nature, 2020. 578(7793): p. 166-171.

252. Wang, X., et al., Regulation of histone methylation by automethylation of PRC2. Genes Dev, 2019. 33(19-20): p. 1416-1427.

253. Lowe, B.R., et al., Histone H3 Mutations: An Updated View of Their Role in Chromatin Deregulation and Cancer. Cancers (Basel), 2019. 11(5).

254. Lee, C.H., et al., Automethylation of PRC2 promotes H3K27 methylation and is impaired in H3K27M pediatric glioma. Genes Dev, 2019. 33(19-20): p. 14281440.

255. Takata, H., et al., Chromatin compaction protects genomic DNA from radiation damage. PLoS One, 2013. 8(10): p. e75622.

256. Elia, M.C. and M.O. Bradley, Influence of chromatin structure on the induction of DNA double strand breaks by ionizing radiation. Cancer Res, 1992. 52(6): $\mathrm{p}$. 1580-6.

257. Beckta, J.M., R.S. Bindra, and A.J. Chalmers, Targeting DNA repair in gliomas. Curr Opin Neurol, 2019. 32(6): p. 878-885.

258. Jorgensen, S., G. Schotta, and C.S. Sorensen, Histone H4 lysine 20 methylation: key player in epigenetic regulation of genomic integrity. Nucleic Acids Res, 2013. 41(5): p. 2797-806. 
259. Wang, H., et al., Histone $H 3$ and H4 ubiquitylation by the CUL4-DDB-ROC1 ubiquitin ligase facilitates cellular response to DNA damage. Mol Cell, 2006. 22(3): p. 383-94.

260. Zhang, Y., et al., Histone H3K27 methylation modulates the dynamics of FANCD2 on chromatin to facilitate NHEJ and genome stability. J Cell Sci, 2018. 131(12).

261. Yadav, R.K., et al., Histone H3G34R mutation causes replication stress, homologous recombination defects and genomic instability in S. pombe. Elife, 2017. 6.

262. Puget, S., et al., Mesenchymal transition and PDGFRA amplification/mutation are key distinct oncogenic events in pediatric diffuse intrinsic pontine gliomas. PLoS One, 2012. 7(2): p. e30313.

263. Viswanathan, S.R. and G.Q. Daley, Lin28: A microRNA regulator with a macro role. Cell, 2010. 140(4): p. 445-9.

264. Zhou, J., S.B. Ng, and W.J. Chng, LIN28/LIN28B: an emerging oncogenic driver in cancer stem cells. Int J Biochem Cell Biol, 2013. 45(5): p. 973-8.

265. Hensen, K., et al., Targeted disruption of the murine Plagl proto-oncogene causes growth retardation and reduced fertility. Dev Growth Differ, 2004. 46(5): p. 459-70.

266. Yang, M., et al., Lin28 promotes the proliferative capacity of neural progenitor cells in brain development. Development, 2015. 142(9): p. 1616-27.

267. Piunti, A., et al., Therapeutic targeting of polycomb and BET bromodomain proteins in diffuse intrinsic pontine gliomas. Nat Med, 2017. 23(4): p. 493-500.

268. Yu, J., et al., Reduced H3K27me3 leads to abnormal Hox gene expression in neural tube defects. Epigenetics Chromatin, 2019. 12(1): p. 76.

269. Ginjala, V., et al., BMII is recruited to DNA breaks and contributes to DNA damage-induced H2A ubiquitination and repair. Mol Cell Biol, 2011. 31(10): p. 1972-82.

270. Wu, Z., et al., Polycomb protein EZH2 regulates cancer cell fate decision in response to DNA damage. Cell Death Differ, 2011. 18(11): p. 1771-9.

271. Monje, M., et al., Hedgehog-responsive candidate cell of origin for diffuse intrinsic pontine glioma. Proc Natl Acad Sci U S A, 2011. 108(11): p. 4453-8.

272. Tong, M., et al., The Role of the Slit/Robo Signaling Pathway. J Cancer, 2019. 10(12): p. 2694-2705.

273. Yu, J., et al., The neuronal repellent SLIT2 is a target for repression by EZH2 in prostate cancer. Oncogene, 2010. 29(39): p. 5370-80.

274. Dallol, A., et al., Frequent epigenetic inactivation of the SLIT2 gene in gliomas. Oncogene, 2003. 22(29): p. 4611-6.

275. Filipponi, D., et al., DNA Damage Signaling-Induced Cancer Cell Reprogramming as a Driver of Tumor Relapse. Mol Cell, 2019. 74(4): p. 651-663 e8. 


\section{VITA}

Lee James Pribyl was born in 1987 to Dan and Mary Jo Pribyl. He was raised on a 3rd generation dairy farm in Maple Lake, Minnesota where he graduated from High School in 2006. He attended the University of Minnesota and graduated with a double major in Genetics/Developmental Biology and Microbiology in 2010. From 2010 to 2014 he worked as an assistant scientist in ovarian cancer research at the University of Minnesota. In 2014, he matriculated into the Biomedical Sciences PhD program within the College of Graduate Health Sciences at the University of Tennessee Health Science Center in Memphis, Tennessee. He joined the lab of Dr. Peter J. McKinnon in the Department of Genetics at St. Jude Children's Research Hospital where he completed his dissertation. He anticipates completion of the requirements for his Doctor of Philosophy in 2020 and will then start a post-doctoral fellowship at the Massachusetts Institute of Technology. 